\title{
MULTIPARTICLE PRODUCTION PROCESSES ${ }^{+}$
}

\author{
S. Gasiorowicz*
}

University of Minnesota

Minneapolis, Minn.

This report was prepared as an account of work This report we the United States nor the United States Atomic Energy the United States nor the United States Atomic En any of Commission, nor any of their employees, nor any their contractors, subcontractors, or their employees, makes any warranty, express. or implied, or assumes any legal liability or responsibility for the accuracy, com pleteness or usefulness of any information, apparatius, product or process disclosed, or represents that its use would not infringe privately owned rights.

+ Based on lectures delivered at the University of Minnesota in the spring of 1971

* Supported in part by the U.S. Atomic Energy Commission under Contract No. $\operatorname{AT}(11-1)-1764$ 


\section{DISCLAIMER}

This report was prepared as an account of work sponsored by an agency of the United States Government. Neither the United States Government nor any agency Thereof, nor any of their employees, makes any warranty, express or implied, or assumes any legal liability or responsibility for the accuracy, completeness, or usefulness of any information, apparatus, product, or process disclosed, or represents that its use would not infringe privately owned rights. Reference herein to any specific commercial product, process, or service by trade name, trademark, manufacturer, or otherwise does not necessarily constitute or imply its endorsement, recommendation, or favoring by the United States Government or any agency thereof. The views and opinions of authors expressed herein do not necessarily state or reflect those of the United States Government or any agency thereof. 


\section{DISCLAIMER}

Portions of this document may be illegible in electronic image products. Images are produced from the best available original document. 


\section{I.INTRODUCTION}

At high energies a large fraction of the apparently constant total cross section is inelastic, making up $80-85 \%$ of the total. Multiple particle production is common, and the problem of organization of data is very pressing. Theoretical considerations, no matter how rudimentary, can be very helpful here. Much of the recent theoretical work in this area has dealt with specific models constructed to fit certain deep, fundamental ideas (e.g. the "bootstrap" hypothesis; quantum field theory), and some interesting predictions have emerged from these models. A closer examination of many of these predictions show that they often emerge from a few simple, almost model-independent assumptions. Thus multiperipheral models share factorization of matrix elements and dominance of low momentum transfer, high sub-energy range of variables.

In the following report/certain aspects of present theoretical thinking about multiparticle reactions are presented in this model-independent manner. Section II deals with the kinematics. The multiperipheral point of view is described in section III. Limiting distributions, and in particular the one-particle distribution measured in the "inclusive" process

$$
A+B \rightarrow C+\text { anything }
$$

are discussed in Section IV, while diffractive processes 
form the subject of section V. In the last section VI we discuss speculations about the extension of Reggeism to inclusive reactions, and give a brief, impressionistic, discussion of the promise of a new phenomenology arising out of the identification of inclusive cross sections with discontinuities in thee-particle scattering matrix elements, made by Muelier. Comparison with experimental data is made, whenever possible, but it is a new field that we are discussing, and relatively little is known. It is probably more accurate to describe the report as a preview, rather than a review, and it is certainly not meant to be a complete guide to the theoretical work that has been done on multiparticle reactionst Hopefully it will serve the purpose of providing a framework within which the growing number of experimental results may be evaluated, and a relatively painless introduction to the field for novices.

+ The formidable list of omitted topics includes BetheSalreter type treatments of multiperipherism, bootstrap models, multi-Veneziano dual models, statistical models, partons and partial summations of Feynman graphs. 
II.KINEMATICS

The reaction

$$
A\left(p_{2}\right)+B\left(p_{b}\right) \rightarrow C_{1}\left(k_{1}\right)+C_{2}\left(k_{2}\right)+\cdots+C_{n}\left(k_{n}\right)
$$

is characterized by the matrix element $M\left(p_{a}, p_{b} ; k_{1}, k_{2}, \ldots k_{n}\right)$ in which the dependence on spin is suppressed. The cross section for the reaction is given by ${ }^{+}$

$$
d \sigma=\frac{(2 \pi)^{4}}{4 F_{a b}} \prod_{i=1}^{n} \frac{d^{3} k_{i}}{2 k_{0 i}}\left|M\left(p_{a}, p_{b} ; k_{1} . . k_{n}\right)\right|^{2} \delta\left(p_{a}+p_{b}-\sum_{i=1}^{\infty} k_{i}\right)
$$

where

$$
F_{a b}=\left(\left(p_{a} \cdot p_{b}\right)^{2}-m_{a}^{2} m_{b}^{2}\right)^{1 / 2}
$$

We will find it convenient to introduce the variables ${ }^{++}$

$$
\begin{aligned}
& k_{i}^{ \pm}=k_{i}^{0} \pm k_{i}^{3} \\
& k_{i}=\left(k_{i}^{1}, k_{i}^{2}\right)
\end{aligned}
$$

and write the four-vector as $\mathrm{k}^{\mu}=\left(\mathrm{k}^{+}, \underset{\sim}{\mathrm{k}}, \mathrm{k}^{-}\right)$. The

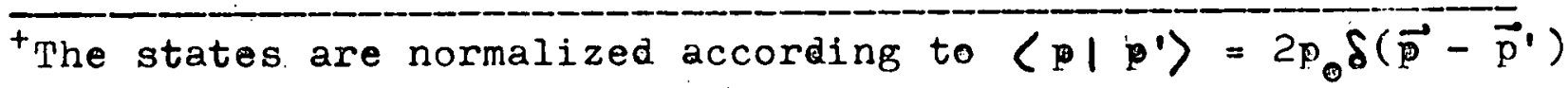

++ These variables, to my knowledge, were first used by Wilson (63); they have proved very useful in quantum electrodynamics, e.f. Chang and Ma (68), Kogut and soper (70) and are related to the better known sudakov variables (Sudakov (1956); Halliday and Saunders (69)). 
mass shell condition

$$
k_{i}^{2}:=k_{i}^{+} k_{i}^{-}-\underset{k_{i}^{2}}{2}=m_{i}^{2}
$$

then implies that

$$
k_{i}^{ \pm}=\frac{m_{+i}^{2}}{k_{i}^{F}}
$$

where we have introduced the convenient notation

$$
m_{j i}^{2}=m_{i}^{2}+k_{i}^{2}
$$

These variables have the advantage that they transform simply under boosts along the 3 -direction. Since

$$
\begin{aligned}
& k^{0^{\prime}}=\cosh \alpha k^{0}+\sinh \alpha^{\prime} k^{3} \\
& k^{3^{\prime}}=\sinh \alpha k^{0}+\cosh \alpha k^{3}
\end{aligned}
$$

where

$$
\cosh \alpha=\left(1-v^{2}\right)^{-1 / 2}
$$

we have

$$
\begin{aligned}
& k^{+^{\prime}}=e^{\alpha} k^{+} \\
& k^{-\prime}=e^{-\alpha} k^{-}
\end{aligned}
$$

and, of course

$$
\underline{k}^{\prime}=\underline{\sim}
$$


$-5-$

We shall find it useful to make use of the following parametrization

$$
\begin{aligned}
& k_{i}^{+}=m_{1 i} e^{y_{i}} \\
& k_{i}^{-}=m_{\perp i} e^{-y_{i}}
\end{aligned}
$$

in terms of which a boost in the 3-direction corresponds to a translation in $y_{1}$

$$
y_{i}^{\prime}=y_{i}+\alpha
$$

Ratios like $k_{i}^{+} / k_{j}^{+}$are invariant under the boosts, as, of course, are the scalar products

$$
k_{i} \cdot k_{j}=\frac{1}{2}\left(k_{i}^{+} k_{j}^{-}+k_{i}^{-} k_{j}^{+}\right)-\underset{\sim}{k_{i}} \cdot \underset{\sim}{k_{j}}
$$

The phase space

$$
d \Phi_{n}=\prod_{i=1}^{n} \frac{d^{3} k_{i}}{2 k_{i}^{0}} \delta\left(p_{a}+p_{b}-\sum_{i=1}^{n} k_{i}\right)
$$

may be rewritten in several ways. If we write

$$
\begin{aligned}
d \Phi_{n}= & \prod_{i=1}^{n} d^{4} k_{i} \delta\left(k_{i}^{2}-m_{i}^{2}\right) \theta\left(k_{i}^{0}\right) \delta\left(p_{a}+p_{b}-\sum_{i=1}^{n} k_{i}\right) \\
= & \prod_{i=1}^{n} \frac{1}{2} d k_{i}^{+} d k_{i}^{-} d^{2}{\underset{\sim}{k}}_{i} \delta\left(k_{i}^{+} k_{i}^{-}-m_{\perp i}^{2}\right) \theta\left(k_{i}^{0}\right) \\
& 2 \delta\left(\sum_{i=1}^{n} \underline{i}_{i}\right) \delta\left(p_{a}^{+}+p_{b}^{+}-\sum_{i=1}^{n} k_{i}^{+}\right) \\
& \delta\left(p_{a}^{-}+p_{b}^{-}-\sum_{i=1}^{n} k_{i}^{-}\right)
\end{aligned}
$$


$-6-$

where the 3-direction is determined by $p_{a}$ when particle $B$ is at rest (the laboratory frame), then carrying out the $\mathrm{dk}_{\mathrm{i}}^{-}$integrations leaves

$$
\begin{aligned}
& d \Phi_{n}=\frac{1}{2^{n-1}} \prod_{i=1}^{n} \frac{d k_{i}^{+}}{k_{i}^{+}} d^{2} k_{i} \delta\left(\sum_{i=1}^{n} \underset{k_{i}}{k_{i}}\right) \delta\left(p_{a}^{+}+p_{b}^{+}-\sum_{i=1}^{n} k_{i}^{+}\right) \\
& \delta\left(p_{a}^{-}+p_{b}^{-}-\sum_{i=1}^{n} \frac{m_{\perp i}^{2}}{k_{i}^{+}}\right)
\end{aligned}
$$

In terms of the "rapidity" variables ${ }^{+} y_{1}$, we have

$$
\frac{d k_{i}^{+}}{k_{i}^{+}}=d y_{i}
$$

and hence

$$
\begin{gathered}
d \Phi_{n}=\frac{1}{2^{n-1}} \prod_{i=1}^{n} d y_{i} d^{2} \underline{k}_{i} \delta\left(\sum_{i=1}^{n} \underset{i}{k_{i}}\right) \delta\left(p_{a}^{+}+p_{b}^{+}-\sum_{i=1}^{n} m_{1 i} e^{y_{i}}\right) \\
\delta\left(p_{a}^{-}+p_{b}^{-}-\sum_{i=1}^{n} m_{\perp i} e^{-y_{i}}\right)
\end{gathered}
$$

In the laboratory frame

$$
\begin{aligned}
& p_{a}=\left(m_{a} e^{Y}, 0, m_{a} e^{-Y}\right) \\
& p_{b}=\left(m_{b}, 0, m_{b}\right) \\
& s \approx m_{a} m_{b} e^{Y}
\end{aligned}
$$

for large $s=\left(p_{a}+p_{b}\right)^{2}$, so that

$$
\begin{gathered}
d \Phi_{n} \approx \frac{1}{2^{n-1}} \prod_{i=1}^{n} d y_{i} d^{2} \underset{k_{i}}{ } \delta\left(\sum_{i=1}^{n} \underset{\sim}{k_{i}}\right) \delta\left(m_{a} e^{Y}-\sum_{i=1}^{n} m_{\perp i} e^{y_{i}}\right) \\
\delta\left(m_{b}-\sum_{i=1}^{n} m_{\perp i} e^{-y_{i}}\right)
\end{gathered}
$$

F The name is due to Feynman (69). The first extensive use of that variable may be found in DeTar (71). 
$-7 \cdot-$

with the last form only valid in the laboratory frame (particle $B$ at rest). To obtain the expression in the projectile frame (particle $A$ at rest) we multiply all the $(+)$ variables by $e^{-Y}$ and the $(-)$ variables by $e^{Y}$ in (2.16).

There is another choice of variables that has proved useful in the multiperipheral approach to multiparticle production. These are the scaled poller variables constructed by Bali, Chew and Pignotti (67). We shall make but little use of them, and therefore limit ourselves to a brief description.

The variables are most easily understood with reference to the multiperipheral graph (Fig. 1). They consist of

(1) a set of $n-1$ "momentum transfers"

$$
\begin{aligned}
& t_{1}=q_{1}^{2}=\left(p_{a}-k_{1}\right)^{2} \\
& t_{2}=q_{2}^{2}=\left(p_{1}-k_{1}-k_{2}\right)^{2} \\
& t_{n-1}=q_{n-1}^{2}=\left(p_{a}-k_{1}-k_{2}-\cdots-k_{n-1}\right)^{2}=\left(p_{b}-k_{n}\right)^{2}
\end{aligned}
$$

(2.19)

(ii) a set of $n-1$ "sub-energy variables":

here a possible choice might be $s_{12}, s_{23}, \ldots s_{n-1, n}$ where, more generally

$$
s_{i j}=\left(k_{i}+k_{i+1}+\cdots+k_{j}\right)^{2}
$$

Actually a better choice from the point of view of 
$-8-$

generalizing the Rage "Ansatz" is to introduce the quantities analogous to the $\cos \theta_{t}$, where $\theta_{t}$ is the center of mass scattering angle in the $t$-channel. In a reaction

$$
\mathrm{A}+\mathrm{B} \rightarrow \mathrm{C}+\mathrm{D}
$$

with (c.m.energy) $)^{2}=s$ and momentum transfer between $C$ and $A$ given by $t$, we have

$$
\cos \theta_{t}=\frac{t(2 s+t-\Sigma)+\left(m_{d}^{2}-m_{b}^{2}\right)\left(m_{c}^{2}-m_{a}^{2}\right)}{\sqrt{\lambda\left(t, m_{a}^{2}, m_{c}^{2}\right)} \sqrt{\lambda\left(t, m_{b}^{2}, m_{d}^{2}\right)}}
$$

$(2.21)$

where

$$
\begin{gathered}
\Sigma=m_{a}^{2}+m_{b}^{2}+m_{c}^{2}+m_{d}^{2} \\
\lambda(x, y, z)=x^{2}+y^{2}+z^{2}-2 x y-2 y z-2 z x
\end{gathered}
$$

$(2.22)$

The analogous variable for the sub-graph describing the scattering of particles described by the momenta

$$
\left(q_{i-1}\right)+\left(-q_{i+1}\right) \rightarrow\left(k_{i}\right)+\left(k_{i+1}\right)
$$

is, in the notation of Bali et al. 
$-9-$

$$
\cosh \xi_{i, i+1}=\frac{t_{i}\left(2 s_{i, i+1}+t_{i}-\Sigma\right)+\left(m_{i}^{2}-t_{i-1}\right)\left(m_{i+1}^{2}-t_{i+1}\right)}{\sqrt{\lambda\left(t_{i}, t_{i-1}, m_{i}^{2}\right)} \sqrt{\lambda\left(t_{i}, t_{i+1}, m_{i+1}^{2}\right)}} .
$$

where

$$
\begin{aligned}
& \Sigma=t_{i-1}+t_{i+1}+m_{i}^{2}+m_{i+1}^{2} \\
& k_{i}^{2}=m_{i}^{2}
\end{aligned}
$$

According to Poller (65) on whose original analysis this work is based, the $\xi_{i, i+1}$ are real and range from 0 to $\infty$, independently of the other variables.

(iii) To complete the total of $3 n-4$ independent variables we need $n-2$ "roller angles", which are in a one-to-one correspondence with the internal vertices in the multiperipheral graph. They are defined by Bali et al. as follows:

Go into the rest frame of $k_{i}$ where the spatial components of the two adjacent momentum transfers point in the same direction. Then consider the rigid rotation about this axis of all momenta standing on the left of the vertex 1 and the independent rigid rotation of all momenta standing on the right. The difference of these two rotation angles is the variable $\omega_{1}$, which has a range 0 to $2 \pi$.

We shall make little use of these variables and 
therefore refer the reader to the papers of Bali et al. for the expression of the phase space in terms of them and for more details that will be of no interest to us. In conclusion, it is useful to get an idea of the magnitudes of some of the quantities which we may want to treat as "large" or "small". With

$$
s=2 M_{p}^{2}+2 M_{p} E_{L}=\left(2 E_{c m}\right)^{2}
$$

we have the following table (in units of $\mathrm{GeV}$ ).

\begin{tabular}{|c|c|c|c|c|}
\hline & $\begin{array}{l}\text { BNL, } \\
\text { CERN } \\
E_{L}=30 \mathrm{GCV}\end{array}$ & SERPUKHOV & NAL & $\begin{array}{c}\text { CERN } \\
\text { ISR } \\
E_{\mathrm{CW}}=30 \mathrm{GeV} \\
\text { Per Proton }\end{array}$ \\
\hline 5 & 58 & 133 & 940 & 3600 \\
\hline$\sqrt{5}$ & 7.6 & 11.6 & 30.7 & 60 \\
\hline $\log 5$ & 4.06 & 4.91 & 6.84 & 8.20 \\
\hline
\end{tabular}

At the same time the range in $\log (\log s)$ is $1.40-2.10$. We mention this only as a reminder that whereas in theory the limit $s \rightarrow \infty$ implies that $\sqrt{s}, \log s$ and $\log (\log s)$ all approach $\infty$, in practice these quantities are fintte and sometimes numerically small. 


$$
-11-
$$

Another quantity of interest is $m_{1}=\left(m^{2}+k^{2}\right)^{1 / 2}$. We have the following table (in units of $\mathrm{GeV}$ ).

\begin{tabular}{|l|l|l|l|l|l|}
\hline & $|k|$ & 0.2 & 0.4 & 0.6 & 0.8 \\
\hline & $\pi$ & 0.06 & 0.18 & 0.38 & 0.66 \\
\cline { 2 - 6 }$m_{L}^{2}$ & $\rho$ & 0.60 & 0.72 & 0.92 & 1.20 \\
\cline { 2 - 6 } & $N$ & 0.92 & 1.04 & 1.24 & 1.52 \\
\hline \multirow{2}{*}{$m_{L}$} & $\rho$ & 0.78 & 0.85 & 0.96 & 1.09 \\
\hline & $N$ & 0.96 & 1.02 & 1.11 & 1.24 \\
\hline
\end{tabular}


III. THE MULTIPERIPHERAL POINT OF VIEW

The early qualitative and sometimes quantitative, successes in explaining the dominant features of twobody cross sections in terms of one-particle (or, later, Regge pole) exchanges, naturally led to the extension of this mechanism to many-particle reactions ${ }^{+}$. For example, the reaction

$$
\pi^{-}+p \rightarrow \pi^{+}+\pi^{-}+n
$$

may be represented by the graph shown in Fig. 2 a. The sub-graph may be viewed as representing pionpion scattering, with one pion off the mass shell and for the outgoing pion sub-energy sufficiently above the resonance region --- so that the quasi-two-body reactions

$$
\pi^{-}+p \rightarrow \rho^{0}\left(f^{0}\right)+n
$$

are excluded, that process may be dominated by a single particle ( $\rho$ ) exchange. Thus the process may be described by the graph shown in Fig. $2 \mathrm{~b}$. The generalization of this to $n$ particles in the final state is shown in fig. 3 . One need not

The extension was independently suggested by Goebel (61) and by Amati, Bertocchi, Fubini, Stanghellini and Tonin (62). The very extensive work of the last set.of authors, known as the ABFST model, was very nicely summarized by Fubini (63). 
restrict oneself to elementary particle exchange; Regge pole exchange is perfectly consistent with the multiperipheral view.

A multiple pion production matrix element represented by fig. 3 will be described by

$$
\begin{aligned}
M= & G_{0}\left(t_{1}\right) \frac{1}{t_{1}-\mu^{2}} M_{n \pi}\left(s_{23}, \theta_{23} ; t_{1}, t_{3}\right) \frac{1}{t_{3}-\mu^{2}} \\
& \cdots M_{n \pi}\left(s_{n-2, n-1}, \theta_{n-2, n-1} ; t_{n-3}, t_{n-1}\right) \\
& \frac{1}{t_{n-1}-\mu^{2}} G_{6}\left(t_{n-1}\right) .
\end{aligned}
$$

Here the $G(t)$ represent form factors and the $M_{n \pi}\left(s_{23}, \theta_{23} ; t_{1}, t_{3}\right)$ etc. are pion-pion scattering matrix elements with the incident pions off shell. Such matrix elements may be taken from experiment, together with some assumption about the effects of going off-shell, or they may be taken from theoretical models that incorporate resonance dominance or Regge pole exchange. ${ }^{+}$In the latter form, presumably both Pomeron and $\rho-f^{\circ}$ contributions should be taken into account. If Regge poles rather than pions are exchanged --- and these could be Pomeron, $\rho$ as well as pion trajectories, consistent with G-parity conservation, an expression

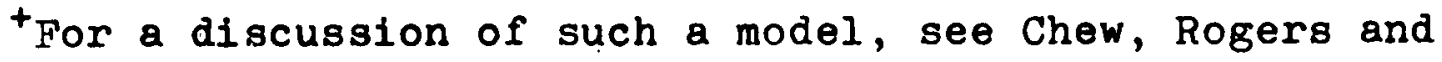
Snider (70). 
of the form

$$
\begin{aligned}
M= & G_{a 1}\left(t_{1}\right)\left(\frac{s_{12}}{s_{0}}\right)^{\alpha_{1}\left(t_{1}\right)} \gamma_{2}\left(t_{1}, t_{2}\right)\left(\frac{s_{23}}{s_{0}}\right)^{\alpha_{2}\left(t_{2}\right)} \\
& \cdots \gamma_{n-1}\left(t_{n-2}, t_{n-1}\right)\left(\frac{s_{n-1, n}}{s_{0}}\right)^{\alpha_{n-1}\left(t_{n-1}\right)}\left(G_{b_{n-1}}\left(t_{n-1}\right)\right.
\end{aligned}
$$

would be appropriate, with the $\gamma\left(t_{1}, t_{i+1}\right)$ describing the coupling of two trajectories to a particle. In obtaining the above form, a Regge Ansatz has been made for the amplitude describing

$$
\text { "Reggeon" + "Reggeon" } \rightarrow \pi+\pi
$$

and the dependence on the Toller angles has been left out. With either iorm it has usually been assumed that It is not necessary to add up all the graphs of the type shown in fig. I that correspond to the many permutations of the outgoing momenta, i.e. that a particular ordering of momenta $1 \mathrm{~s}$ dominant. +

Crucial to the formulation of all multiperipheral points of view are the following assumptions:

The matrix element $M\left(p_{a}, p_{b} ; k_{l}, \ldots k_{n}\right)$ is largest in the region of phase space in which

\section{(1) all the momentum transfers $t_{i}$ are bounded}

This is an important ingredient in the use of the multiperipheral amplitudes to calculate, using unitarity, the two-particle cross section. With a single graph something like an integral equation can be set up, and most "bootstrap" results have been derived from such equations. 
(ii) Subenergies $\mathbf{s}_{i, i+1}$ or cluster subenergies are large, and

the matrix element in this kinematical region has certain factorization properties.

All of these statements need detailed discussion. +

(i) It is well known that two-particle cross sections show a strong decrease with increasing $t$ : for elastic scattering the dependence ${ }^{++}$

$$
\frac{d \sigma}{d t}=f(s) e^{a t}
$$

with $a \approx 6-8(\mathrm{GeV} / \mathrm{c})^{-2}$ is typical; for inelastic twobody reactions the behavior is

$$
\frac{d \sigma}{d t}=G(t) s^{2 \alpha(0)-2} e^{2\left(\alpha^{\prime}(0) \log s\right) t}
$$

where $\alpha^{\prime}(0) \approx I(\mathrm{GeV} / \mathrm{c})^{-2}$ and $G(t)$ is a relatively slowly varying function of $t$. It seems natural to extend this kind of dependence to each subgraph in the multiperipheral chain, which describes the scattering of two off-shell objects ( "virtual particles" or "Reggeons") to yield two real particles. This assumption leads to a prediction: the transverse momenta $\left|\mathrm{k}_{i}\right|$ of the pro-

Tuch of what follows is discussed by Zachariasen and Zweig (67).

$+{ }^{+}$All the $t_{i}$ are spacelike. In the rest frame of $B$ all the $q_{i}^{0}>0$ for energy conservation; in the rest frame of $A$ all the $q_{i}^{0}<0$ for the same reason. This is only possible if the $q_{i}$ are spacelike, i.e. the $t_{i}<0$. 
$-16-$

duce particles are also bounded.

Let us work in the center of mass frame. At high energies we may take

$$
\begin{aligned}
& p_{a} \approx\left(\sqrt{s}, 0, m_{a}^{2} / \sqrt{s}\right) \\
& p_{b} \approx\left(m_{b}^{2} / \sqrt{5}, 0, \sqrt{5}\right)
\end{aligned}
$$

If we write

$$
\begin{aligned}
& Q_{1}=k_{1}+k_{2}+\ldots+k_{i} \\
& Q_{2}=k_{i+1}+\ldots+k_{n} . \\
& Q_{1}^{2}=M_{1}^{2} ; Q_{2}^{2}=M_{2}^{2} .
\end{aligned}
$$

then momentum conservation leads to

$$
\begin{gathered}
Q_{1}+Q_{2}=0 \\
\sqrt{5} \approx Q_{1}^{+}+Q_{2}^{+}=Q_{1}^{+}+\left(M_{2}^{2}+Q_{2}^{2}\right) / Q_{2}^{-} \\
\sqrt{5} \approx Q_{1}^{-}+Q_{2}^{-}=\left(M_{1}^{2}+Q_{1}^{2}\right) / Q_{1}^{+}+Q_{2}^{-}
\end{gathered}
$$

Note that $Q_{1}^{ \pm}, Q_{2}^{ \pm}>0$. Now

$$
\begin{aligned}
t_{i}=\left(Q_{1}-p_{a}\right)^{2} & =M_{1}^{2}+m_{a}^{2}-p_{a}^{+} Q_{1}^{-}-p_{a}^{-} Q_{1}^{+} \\
& =M_{1}^{2}+m_{a}^{2}-\sqrt{5} \frac{M_{1}^{2}+Q_{1}^{2}}{Q_{1}^{+}}-\frac{m_{a}^{2}}{\sqrt{5}} Q_{1}^{+} \\
& =m_{a}^{2}\left(1-\frac{Q_{1}^{+}}{\sqrt{5}}\right)-M_{1}^{2}\left(\frac{\sqrt{5}}{Q_{1}^{+}}-1\right)-\frac{\sqrt{5}}{Q_{1}^{+}} Q_{1}^{2}
\end{aligned}
$$

Since $Q_{1}^{+}>0$ and $\sqrt{s} / Q_{1}^{+}>1$, the first term is 
bounded. The other two terms are both negative, as is $t_{i}$, and hence the boundedness of $t_{i}$ implies the boundedness of the two terms separately. From this we deduce that $\sqrt{s} / Q_{1}^{+}$cannot get very large, $M_{1}^{2}$ can only get large if $\sqrt{\mathrm{s}} / \mathrm{Q}_{1}^{+} \longrightarrow 1$, and $Q_{1}^{2}$ must be bounded. Thus $\left|\sum_{r=1}^{i} k_{I}\right|$ is bounded for all 1 , and hence all the $\left|k_{i}\right|$ ane bounded.

The experimental situation is that the $\left|\mathbf{k}_{i}\right|$ are indeed bounded, with

$$
\left\langle k_{\perp}\right\rangle \equiv\langle|\underline{\sim}|\rangle \approx 0.3-0.4 \mathrm{GeV} / \mathrm{c} \text { (3.9) }
$$

This average value is almost independent of particle species, though it may be increasing slightly with the mass of the particle; at cosmic ray energies it may be increasing slowly, perhaps like $\log \mathrm{s}$, and it increases slowly. with the multiplicity at a given energy ${ }^{+}$. THis, almost universal limitation on the transverse momentum is perhaps the most striking characteristic of high energy reactions.

It is interesting to note that the two body t-dependence, $e^{\text {at }}\left(a=6-8(\mathrm{GeV} / \mathrm{c})^{2}\right)$ translates into ${ }^{++}$

$$
\left\langle k_{\perp}\right\rangle=\frac{\int d \Omega e^{a t}(p \sin \theta)_{\mathrm{cm}}}{\int d \Omega e^{a t}} \approx 0.32-0.34 \mathrm{GeV} / \mathrm{c}
$$

+ See, for example Turkot (68) or Elbert et al. (68) ${ }^{++}$See, for example Quige (71). 
Fig. 4 shows a typical distribution of transverse momenta. (ii) Let us next consider the statement:"subenergies $s_{i, i+1}$ are large" and see what its implications are.

For any adjacent pair of outgoing momenta

$$
\begin{aligned}
s_{i, i+1}=\left(k_{i}+k_{i+1}\right)^{2}=m_{i}^{2}+m_{i+1}^{2} & +\left(k_{i}^{+} k_{i+1}^{-}+k_{i}^{-} k_{i+1}^{+}\right) \\
& -2 k_{i} \cdot k_{i+1}
\end{aligned}
$$

For this to be large, with bounded transverse momenta, it follows from

$$
\begin{aligned}
& k_{i}^{+} k_{i+1}^{-}=m_{\perp(i+1)}^{2}\left(k_{i}^{+} / k_{i+1}^{+}\right) \\
& k_{i}^{-} k_{i+1}^{+}=m_{\perp i}^{2}\left(k_{i+1}^{+} / k_{i}^{+}\right)
\end{aligned}
$$

that either $k_{i}^{+} / k_{i+1}^{+}$or $k_{i+1}^{+} / k_{i}^{+}$must be much larger than 1 . Now the boundedness of

$$
t_{1}=\left(p_{a}-k_{1}\right)^{2}=m_{a}^{2}+m_{1}^{2}-\left(\sqrt{5}-k_{1}^{+}\right)\left(\frac{m_{a}^{2}}{\sqrt{5}}-\frac{m_{11}^{2}}{k_{1}^{+}}\right)
$$

implies that $k_{1}^{+} \sim O(\sqrt{5})$, and this means that $k_{2}^{+}$cannot be much larger than $k_{l}^{+}$. Making use of the boundedness of the $t_{i}$ we can thus show that $s_{i, i+1} \gg 1$ (in units of $(\mathrm{GeV})^{2}$, say) implies that

$$
k_{i}^{+} / k_{i+1}^{+}>>1
$$


This is described as strong ordering. ${ }^{+}$

In the strong ordering limit the form of the matrix element (3.2) becomes very plausible. The matrix element may, with the help of

$$
S_{i, i+1} \approx m_{\perp(i+1)}^{2} \frac{k_{i}^{+}}{k_{i+1}^{+}}
$$

be written in the form

$M=f_{n}\left(k_{1}, k_{2} \ldots k_{n}\right)\left(\frac{k_{1}^{+}}{k_{2}^{+}}\right)^{\alpha_{1}\left(t_{1}\right)}\left(\frac{k_{2}^{+}}{k_{3}^{+}}\right)^{\alpha_{2}\left(t_{2}\right)} \ldots\left(\frac{k_{n-1}^{+}}{k_{n}^{+}}\right)^{\alpha_{n-1}\left(t_{n-1}\right)}$

which shows very clearly the factorization property of the matrix element. This form satisfies the formulation of the factorization hypothesis given by Wilson (63) many years ago:

Particles that are relativistic to each other (and this includes the incident particles) have their momenta $k_{i}^{+}$appear in separate factors in expressions that must be manifestly inva-

\footnotetext{
+ Strong ordering appears to follow from dynamical arguments; Fubini (63) obtains it by (i) maximizing the one-pion exchange matrix element $\prod_{i}\left(t_{1}-\mu^{2}\right)^{-1}$ subject to the mass shell constraints, and (ii) by assuming that $\left(s / \mu^{2}\right)^{\frac{1}{n+1}} \gg 1$. The second requirement, as will become clear, is very similar to the requirement that all the subenergies be large.
} 
riant under boosts along the $z$ axis. Using only this very general statement of factorization and the strong ordering kinematics, a number of conclusions can be drawn.

Momentum conservation in the center of mass frame

$$
\begin{aligned}
& \sqrt{5} \approx k_{1}^{+}+k_{2}^{+}+\cdots+k_{n}^{+} \\
& \sqrt{5} \approx k_{1}^{-}+k_{2}^{-}+\cdots+k_{n}^{-}
\end{aligned}
$$

and the condition $(3.11)$ imply that

$$
k_{1}^{+} \approx \sqrt{s}=p_{a}^{+}
$$

and, similarly

$$
k_{n}^{-} \approx \sqrt{s}=p^{-}
$$

We call the particles with momenta $k_{1}$ and $k_{n}$ leading particles, and we may estimate the fraction of the energy carried off by them in the following way:

Let the ratio

$$
k_{i}^{+} / k_{i+1}^{+}=r
$$

be the same for all i so that 


$$
\begin{aligned}
\frac{\sqrt{5}}{k_{1}^{+}} & =1+\frac{k_{2}^{+}}{k_{1}^{+}}+\frac{k_{3}^{+}}{k_{2}^{+}} \cdot \frac{k_{2}^{+}}{k_{1}^{+}}+\cdots+\frac{k_{n}^{+}}{k_{n-1}^{+}} \cdot \frac{k_{n-1}^{+}}{k_{n-2}^{+}} \cdots \frac{k_{2}^{+}}{k_{1}^{+}} \\
& =1+\frac{1}{r}+\frac{1}{r^{2}}+\cdots+\frac{1}{r^{n-1}} \\
& \approx \frac{1}{1-(1 / r)}
\end{aligned}
$$

Hence

$$
\frac{k_{1}^{+}}{\sqrt{5}}=1-\frac{1}{r}
$$

where $r$ is supposed to be large. It may be estimated in the following way: we write

$$
\begin{aligned}
s=m_{b}^{2} \frac{p_{a}^{+}}{p_{b}^{+}} & =m_{b}^{2} \frac{p_{a}^{+}}{k_{1}^{+}} \cdot \frac{k_{1}^{+}}{k_{2}^{+}} \cdots \frac{k_{n-1}^{+}}{k_{n}^{+}} \frac{k_{n}^{+}}{p_{b}^{+}} \\
& =m_{b}^{2} \frac{1}{1-\frac{1}{r}} \cdot r^{n-1} \cdot \frac{m_{1 n}^{2}}{m_{b}^{2}} \frac{p_{b}^{-}}{k_{n}^{-}} \\
& =m_{\perp n}^{2} \frac{r^{n-1}}{(1-1 / r)^{2}}
\end{aligned}
$$

Thus, very roughly ${ }^{+}$

\footnotetext{
The condition for strong ordering obtained by Pubini (63) (see footnote on p. 19 ) is, according to eq. (3.19) just the condition that $r$ be much larger than 1 .
} 


$$
r \approx\left[\frac{s}{\left\langle m_{i}^{2}\right\rangle}\right]^{\frac{1}{n-1}}
$$

There is consequently a relation between $r, s$ and the number of particles produced

$$
\bar{n}=\frac{\log s}{\log r}+\text { const }
$$

Since a typical subenergy in the middle of the multiperipheral chain is

$$
s_{i, i+1} \cong m_{1(i+1)}^{2} \frac{k_{i}^{+}}{k_{i+1}^{+}} \approx m_{1}^{2} r
$$

we may use the general notion of factorization, with its implication that what goes on in the middle of the chain is decoupled from the ends, to argue that $\overline{s_{i, i+1}}$ and hence $r$ should be independent of $s$. Thus the multiperipheral point of view strongly suggests a $\log s$ dependence for the multiplicity.

A recent cosmic-ray experiment on p-p interactions between 90 and $800 \mathrm{GeV}$ (Jones et al (70)) yielded ${ }^{+}$

$$
\bar{n} \approx(1.07 \pm .15) \log 5+\text { const. }
$$

T The experiment yields the multiplicity of charged particles, which we have multiplied by $3 / 2$. This number is subject to some uncertainties. For further discussion see p. 26 . 
This would imply that

$$
r=2.4-2.9
$$

which could hardy be called "very large".

In terms of the rapidity variables. $y_{1}$ introduced in $(2.11)$ and $(2.17)$, eq. $(3.20)$ reads

$$
\bar{n}=\frac{\dot{Y}}{\log r}+\text { const } .
$$

i.e. $\log r$ is the average spacing between the $y$ values of the particles. Factorization, i.e. decoupling of particles is associated with large $r$, i.e. large spacing in $\mathrm{y}$, and the multiplici-ty measurements indicate that the average spacing is not very large. There is more evidence along the same lines. An analysis of the reaction

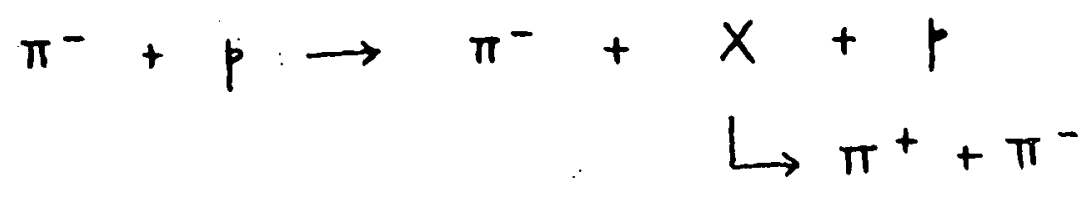
at $25 \mathrm{GeV}$ by Lipes et al. (69) shows that events with $s_{X \pi} \geqslant 2 \mathrm{GeV}^{2}$ and $s_{X p} \geqslant 4 \mathrm{GeV}^{2}$, further constrained to eliminate events in which one of the pions from $X$ resonates with either the final proton or $\pi^{-}$, and constrained to the low momentum transfer region (the cut $\left|t_{p}+2 t_{\pi}\right|<0.8$ was made) form only about $12 \%$ of the total four-prong events. Thus at this energy with this multiplicity, events with large subenergies are rare. 
Finally, it is reported that if final particles from a multiple-production reaction are ordered according to the $k_{z}$, then the mean subenergy of a neighboring pion pair is less than, or of the order of $1 \mathrm{GeV}$. $^{+}$These observations can be met as follows:

(i) One may decide to concentrate on the large subenergy region, even though there are not many events in it, and test Multi-Regge exchange theory ( as exemplified by (3.2) ) there.

(ii) Ore may decide to use (3.2) or more sophisticated variants thereof outside of its strict region of applicability, by appealing to duality. This has proved quite successful in some cases, e.g. In the analysis of

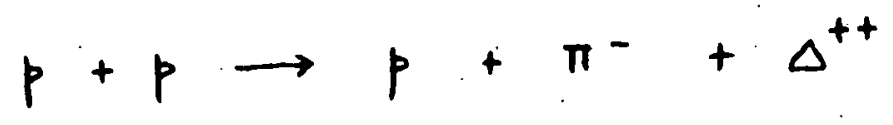

at $28.5 \mathrm{GeV} / \mathrm{c}$. (Berger (69))., and in other reactions, reviewed very carefully by Ranft (70), but the appeal to duality leaves one uneasy in reactions involving primarily pions. Fig. 5. from the review by Fox (69) indicates the discrepancy between resonant amplitudes and Regge amplitudes in pion-pion scattering. (iii) One may conclude, as we do, that the matrix

The report by. Z.Ajduk, I. Michejda and W.Wojcik is referred to in Chow et al (70). I have not seen the paper; whatever the energy range and multiplicities considered, the energy is presumably no larger than $25 \mathrm{GeV}$, i.e. that these results confirm and extend the analysis by Lipes et al. quoted earlier. 
element $M\left(p_{a} ; p_{b} ; k_{1}, \ldots k_{n}\right)$ is factorizable, but not necessarily in terms of two-particle scattering amplitudes, but perhaps in terms of products of amplitudes of the form

$$
(\text { Particle })_{1}+(\text { particle })_{2} \longrightarrow(\text { cluster })_{1}+(\text { cluster })_{2}
$$

Such clusters may or may not be resonances.

The fact that clusters must occur at higher energies

follows from kinematics: in the center of mass frame we have

$$
\begin{aligned}
& \sqrt{5} \cong k_{1}^{+}+k_{2}^{+}+\cdots+k_{n}^{+} \\
& \sqrt{5} \cong k_{1}^{-}+k_{2}^{-}+\cdots+k_{n}^{-}
\end{aligned}
$$

Suppose we order the $\mathrm{k}_{i}^{+}$so that

$$
k_{1}^{+}>k_{2}^{+}>\cdots>k_{n}^{+}
$$

and for each event plot the values of $k_{1}^{+} / k_{2}^{+}, k_{2}^{+} / k_{3}^{+}$, $\ldots . . . k_{n-1}^{+} / k_{n}^{+}$the following things can happen:

(i) all of the ratios are of the same order of magnitude; if all of the $k^{+}$'s are small (of order l, say) then there must be many of them, leading to multiplicities of order $\sqrt{\mathbf{s}}$. This is not observed. All the $k^{+}$'s cannot be large, however, since if they were, all of the $k^{-}$'s would be small, again in conflict with (3.25). (ii) There is a number of breaks in the sequence of ratios. The particles with momenta for which the ratios 
are of order 1 , form a cluster; the next group after the break again form a cluster, and so on. Fig. 6 shows the clustering corresponding to

$$
\begin{aligned}
& k_{1}^{+} / k_{2}^{+}, k_{2}^{+} / k_{1}^{+}, \ldots k_{l-1}^{+} / k_{l}^{+} \sim O(1) \\
& k_{l}^{+} / k_{l+1}^{+} \gg 1 \\
& k_{l+1}^{+} / k_{l+2}^{+}, \ldots k_{r-1}^{+} / k_{r}^{+} \sim O(1) \\
& k_{r}^{+} / k_{r+1}^{+} \gg 1 \\
& k_{r+1}^{+} / k_{r+2}^{+}, \ldots \ldots k_{n-1}^{+} / k_{n}^{+} \sim O(1)
\end{aligned}
$$

Given the clustering, there are again two possibilities: (a) the number of clusters remains fixed as the energy increases, or

(b) the number of clusters grows as the energy increases, perhaps like log s.

There is also the possibility that there are two contributıons to the matrix element: one, which we call "diffractive" which has one or two clusters, the other, multiperipheral, which has a growing number of clusters.

If we assume that the $\pi^{\prime} s$ and $K^{\prime} s$ in the cosmic ray data come from clusters (or resonances) that produce 2 3 particles each, on the average, then the number of clusters will be, according to (3.22)

\section{$\bar{n} \approx 0.4 \log s+$ const.}


and hence for the clusters,

$$
r \approx 12
$$

which is certainly large enough to be consistent with the starting premise. In terms of the $y$ variables, we have pairs or triplets of points that are now more widely separated in $\mathrm{y}$.

A relation like ( 3.18$)$, written in terms of cluster momenta, shows that the leading clusters carry off $\geq 90 \%$ of the energy. If these consist of $2-3$ particles, it is not unreasonaole to expect that $50-60 \%$ of the energy should be carried of by the most energetic of them. This is indeed what is observed.

The multiperipheral point of view also allows some conjectures regarding the distribution of multiplicities about the mean. The simplest conjecture, based on the strong ordering limit in which all particles are uncorrelated, is that of a Poisson distribution. The probability of finding $n$ particles is given by

$$
P_{n}=e^{-\lambda} \frac{\lambda^{n}}{n !}
$$

The factor in front ensures that $\sum_{n} P_{n}=1$; the relative probability of finding $n$ particles is $\lambda^{n} / n$ ! with the denominator factor present because we are considering only one species of particles. The significance of $\lambda$ 
$-28-$

is seen from

$$
\bar{n}=\sum_{n} n P_{n}=e^{-\lambda} \sum_{n} \frac{n \lambda^{n}}{n !}=\lambda
$$

If we believe that all particles are uncorrelated, then charged pions, which are actually the particles detected ${ }^{+}$, will also obey a Poisson distribution, with

$$
\lambda=\bar{n}_{c} \simeq 0.7 \log 5+2.2
$$

according to Jones et al. (70). The values of $\lambda$ are 5 and 7 at BNL-CERN and NAL energies, respectively. The table below lists $P_{n}$ corresponding to these values of $\lambda$, and gives us an idea of the probability of large fluctuations about the mean value.

\begin{tabular}{rcc} 
& \multicolumn{2}{c}{ Values of $\mathrm{P}_{\mathrm{n}}$} \\
$\mathrm{n}$ & $\lambda=5$ & $\lambda=7$ \\
\hline 4 & 0.176 & $9.1 \times 10^{-2}$ \\
6 & 0.145 & .15 \\
8 & $6.5 \times 10^{-2}$ & .13 \\
10 & $1.8 \times 10^{-2}$ & $7.1 \times 10^{-2}$ \\
12 & $3.1 \times 10^{-3}$ & $2.6 \times 10^{-2}$ \\
14 & $4.2 \times 10^{-4}$ & $7.1 \times 10^{-3}$ \\
16 & - & $1.5 \times 10^{-3}$ \\
18 & - & $2.3 \times 10^{-4}$
\end{tabular}

$+\mathrm{K}$ production appears to be small. See, for example Abolins et al.(70), Diddens et al.(64) and Rant (70), all of which indicate $\leqslant 5 \%$ effects. 
Comparison with $25 \mathrm{GeV}$ data of Elbert et al. shows that the ratios are in reasonable agreement the Poisson distribution.

\begin{tabular}{llllllll}
\hline No. of charged & 4 & 6 & 8 & 10 & 12 & 14 \\
\hline $\begin{array}{l}\text { tracks } \\
\sigma_{\mathrm{n}} \text { (mb) from }\end{array}$ & 4.0 & 4.7 & 1.7 & 0.6 & .09 & .01 \\
Elbert et al. & 7.09 \\
\hline $\begin{array}{l}\text { Poisson distr. } \\
\begin{array}{l}\lambda=5) \text { normal } \\
\text { at } \mathrm{n}_{\mathrm{c}}=4\end{array}\end{array}$ & 7.0 & 5.8 & 2.6 & 0.7 & .12 & .02 \\
\hline
\end{tabular}

It is clear that a pure Poisson Distribution represents something of an oversimplification, since (a) it does not take into account charge conservation, or the fact that multiperipheral events are more likely to occur in clusters, and (b) the experimental evidence from Elbert et al.(70) indicates that neutral pions do not form with $\bar{n}_{x^{\circ}} \sim 1 / 2 \bar{n}_{c}$. Rather, the trend is that $2-3$ $\pi^{\circ}$ are formed as the number of charged tracks varies from $4-14$, i.e. $\pi^{\circ}$ production is not completely independent. It is likely that once charge conservation and multiperipheral resonance production are taken into account, the $\pi^{\circ}$ data will also be explained. ${ }^{+}$

Fodified Poisson distributions that take these into account have been studied by Horn and Silver and by Caneschi and schwimmer (71), with encouraging results. 
IV. LIMITING DISTRIBUTIONS

Experience with two-particle reactions teaches us that cross sections have a varlety of $s$-dependences. Diffractive processes ${ }^{+}$remain constant as $s$ grows; processes in which an exchange of charge, i-spin, baryon number, etc. takes place, tend to fall off like $s^{2 \alpha(0)-2}$ where $\alpha(0)$, the intercept of the leading trajectory carrying the appropriate quantum numbers, always $\leqslant 1 / 2$. Thus at high energies many cross sections become very tiny, and it is important to identify quantities that approach nonvanishing limits as $s \rightarrow \infty$. This cannot be done in a model independent way.

Benecke, Chou, Yang and Yen (69) hypothesize that what happens in a collision of two hadrons is that the hadrons shatter into fragments. In the laboratory frame, the fragments of the projectile will have momenta that are fractions of the initial momentum of the projectile. Since the fragment momenta increase without limit as $s \rightarrow \infty$, any distribution of momenta of fragments of the projectile will not

F For further discussion see Sec.v.

++ The prediction from Regge theory is that $d \sigma / d t \simeq c(t) 5^{2 \alpha(t)-2}$ With observed linear trajectories $\alpha(t)=\alpha(0)+\alpha^{\prime} t$ and relatively smooth $c(t)$, the integration over $t$, be it the kinematically allowed range $-s<t<0$, or a finite range $-\tau<t<0$, leads to the behavior $s^{2 \alpha(0)-2}$ within factors of $\log \mathrm{s}$. 
have a limit in the laboratory frame. The target will also fragment. In the laboratory frame the target "sees" a very thin disk of hadronic matter hitting it; as the energy increases, the disk gets thinner, but as soon as the thickness is small compared to the size of the relevant hadronic bits that interact, the energy increase will no longer affect the target. Hence in the laboratory frame, momentum distributions of the target fragments approach a limit as $s \rightarrow \infty$. Similarly, in the rest frame of the projectile, momentum distributions of the projectile fragments approach a limit as $s \rightarrow \infty$.

If we compare this picture with that of the multiperipheral model, we see that what BCYY are talking about are the clusters at the two ends of the multiperipheral chain. In the latter model, however, the bulk of the multiple production comes from the middle of the chain. The fragmentation point of view can accommodate such an effect (Fig. 7 b ); BCYY following earlier terminology refer to this as the pionization component. Roughly speaking, in terms of the discussion following eq. (3.26) the existence of a single break in the ratio of the $k^{+}$is corresponds to fragmentation alone, while two breaks would describe a sharply differentiated pionization component. At finite energies the separation of momenta into the three classes: projectile fragments, $k_{1}^{+} \sim 0(s)$, pionization products, $k_{i}^{+} \sim 0(\sqrt{s})$, target fragments, 
$k_{i}^{+} \sim 0(1)$ becomes more difficult, since it is impossible to distinguish between a particularly slow projectile fragment and a particularly fast target fragment. We will see later how this difficulty can, partially, be overcome.

The distribution most easily measured is the oneparticle distribution. This is obtained by integrating over all momenta but one, and summing over the number of particles produced. If we write eq. (2.1) in the laboratory frame

$$
\begin{aligned}
d \sigma^{(n)}\left(k_{1} \ldots k_{n}\right) \approx & \frac{(2 \pi)^{4}}{2 s} \prod_{i=1}^{n} \frac{d k_{i}^{+}}{2 k_{i}^{+}} d^{2} \underset{\sim}{k_{i}} \delta\left(\sum \underset{\sim}{k_{i}}\right) \\
& \delta\left(s / m_{b}-\sum k_{i}^{+}\right) \delta\left(m_{b}-\sum k_{i}^{-}\right)\left|M\left(p_{a}, p_{b} ; k_{1} . . k_{n}\right)\right|^{2} \\
\equiv & \prod_{i=1}^{n} \frac{d k_{i}^{+}}{k_{i}^{+}} \stackrel{d^{2}}{\sim} k_{i} \delta\left(\sum \underset{\sim}{k_{i}}\right) \delta\left(\frac{s}{m_{b}}-\sum k_{i}^{+}\right) \\
& \delta\left(m_{b}-\sum k_{i}^{-}\right)|\bar{M}|^{2}
\end{aligned}
$$

then

$$
\begin{aligned}
d \sigma^{(n)}(k)= & \frac{d k^{+}}{k^{+}} d^{2} \underset{\sim}{k} \frac{1}{(n-1) !} \int_{i=1}^{n-1} \frac{d k_{i}^{+}}{k_{i}^{+}} d^{2} \underline{\sim}_{i} \delta\left(\sum_{\sim}^{k}+\sum_{i=1}^{n-1} k_{i}\right) \\
& \delta\left(\frac{s}{m_{b}}-k^{+}-\sum_{i=1}^{n-1} k_{i}^{+}\right) \delta\left(m_{b}-k^{-}-\sum_{i=1}^{n-1} k_{i}^{-}\right) \\
& \left|\bar{M}\left(p_{0}, p_{b i} k k_{1} \ldots k_{n-1}\right)\right|^{2}
\end{aligned}
$$

gives the cross section for finding a particle with momentum $k$, given that there are $n$ particles in the final state. The factor $\frac{1}{(n-1) !}$ appears when the particles that are integrated over are identical, and the reason for it is the following: 
if the particles are all identical, then $M$ is a symmetric function of all the ' $k^{\prime} s$. To associate the variables $k$ with particles; we must make a definite rule, e.8. that ${ }^{+}$

$$
k_{1}^{+}>k_{2}^{+}>\cdots>k_{n}^{+}
$$

In the phase space integration in (4.2) we may either follow this defintion in the limits of integration, or, more conveniently, we integrate all momenta over all of phase space, and take multiple counting into account by dividing by $(n-1) !$. The distribution of interest, measured in a one-arm spectrometer experiment of the type

$$
A+B \rightarrow C(k)+\text { "anything" }
$$

is given by

$$
\sum_{n=2} d \sigma^{(n)}(k) \equiv \frac{d k^{+}}{k^{+}} d^{2} \underset{\sim}{k} p_{1}\left(k^{+}, k ; s\right)
$$

The hypothesis of BCYY is that

$$
\rho_{1}\left(k^{+}, k ; j\right)=\sum_{n=2} \frac{1}{(n-1) !} \int_{i=1}^{n-1} \frac{d k_{i}^{+}}{k_{i}^{+}} d^{2} k_{i} \delta \ldots|\bar{M}|^{2}
$$

This has nothing to do with "strong ordering", which is a statement about the matrix element, but merely tells us which momentum we will call $\mathrm{k}_{1}$, which $\mathrm{k}_{2}, \ldots$ If there are several. species of particles we may use the labeling $k_{i \pi}, k_{i k}, k_{i p}, \ldots$ and we will get factorials $\left(n_{n}-1\right) ! n_{k} ! \ldots$ in the denominator. 
$-34-$

approaches a limit as $s \rightarrow \infty$, and that the limit describes the one-particle fragmentation distribution of the target.

The mean multiplicity of fragments may be expressed in terms of this distribution function:

$$
\begin{aligned}
\bar{n} & =\frac{\sum_{n} n \frac{1}{n !} \int \prod_{i=1}^{n} \frac{d k_{i}^{+}}{k_{i}^{+}} d^{2} k_{i} \delta \ldots|\bar{M}|^{2}}{\sum_{n} \frac{1}{n !} \int \prod_{i=1}^{n} \frac{d k_{i}^{+}}{k_{i}^{+}} d^{2} k_{i} \delta \ldots|\bar{M}|^{2}} \\
& =\frac{\sum_{n} \frac{1}{(n-1) !} \int \frac{d k^{+}}{k^{+}} d^{2} \underset{\sim}{k} \int \prod_{i=1}^{n-1} \frac{d k_{i}^{+}}{k_{i}^{+}} d^{2} k_{i} \delta \ldots|M|^{2}}{\sigma_{+o t}(s)} \\
& =\frac{\int \frac{d k^{+}}{k^{+}} d^{2} \underset{\sim}{k} \rho\left(k^{+}, k, s\right)}{\sigma_{\text {tot }}(s)}
\end{aligned}
$$

The delta function $\delta\left(m_{b}-k^{-}-\sum_{i=1}^{n-1} k_{i}^{-}\right)$in (4.1) shows that, since all the $k_{i}^{-}>0$,

$$
k^{-}=m_{b}-\sum_{i=1}^{n-1} k_{i}^{-}<m_{b}
$$

If the particles "l", "2" , .. "nil" that are not picked up by the spectrometer are all fragments of the projectile, then $k_{1}^{-} \rightarrow 0$ (like $1 / s$ ) and $k^{-}$ approaches the kinematic boundary

$$
k^{-}=m_{6}
$$


$-35-$

The boundary is a parabola in the $k_{\perp}=|\underset{\sim}{\mid}|$ and $k_{z}$ plane

$$
2 m_{b} k_{z}=k_{\perp}^{2}+m_{c}^{2}-m_{b}^{2}
$$

If we look at two-particle distributions, we see that there are two contributions: from reactions in which just two particles are produced

$$
\begin{aligned}
d \sigma^{(2)}\left(k, k^{\prime}\right)= & \frac{d k^{+}}{k^{+}} \frac{d k^{\prime+}}{k^{\prime+}} d^{2} \underset{\sim}{k} d^{2} \underline{k}^{\prime} \delta\left(\underset{\sim}{k}+\underline{k}^{\prime}\right) \\
& \delta\left(\frac{s}{m_{b}}-k^{+}-k^{\prime+}\right) \delta\left(m_{b}-k^{-}-k^{\prime}\right)\left|\bar{M}_{2}\right|^{2} \\
\equiv & \frac{d k^{+}}{k^{+}} \frac{d k^{\prime+}}{k^{\prime+}} d^{2} \underset{k}{k} d^{2} \underline{k}^{\prime} \bar{\sigma}_{2}\left(k, k^{\prime} ; s\right)
\end{aligned}
$$

and from reactions in which more particles are produced, but two are singled out

$$
\begin{aligned}
\sum_{n=3} d \sigma^{(n)}\left(k, k^{\prime}\right)= & \frac{d k^{+}}{k^{+}} \frac{d k^{\prime+}}{k^{\prime+}} d^{2} k d^{2} k^{\prime} \\
& \sum_{n=2} \frac{1}{(n-2) !} \int \prod_{i=1}^{n-2} \frac{d k_{i}^{+}}{k_{i}^{+}} d^{2} \underline{\sim}_{i} \delta \ldots\left|\bar{M}_{n}\right|^{2} \\
\equiv & \left.\frac{d k^{+}}{k^{+}} d^{2} \underset{k^{\prime}}{\frac{d k^{\prime}}{k^{\prime}}} d^{2} k^{\prime} \bar{\tau}_{2} \mid k, k^{\prime} ; s\right)
\end{aligned}
$$

BCYY conjecture that

$$
\rho_{2}\left(k, k^{\prime} ; s\right)=\bar{\sigma}_{2}\left(k, k^{\prime} ; s\right)+\bar{\tau}_{2}\left(k, k^{\prime} ; s\right)
$$




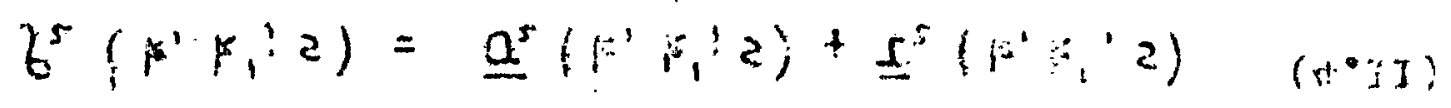

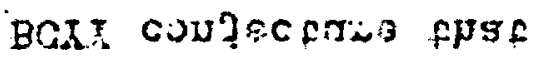

$$
\begin{aligned}
& \text { (it. } \mathrm{JO})
\end{aligned}
$$

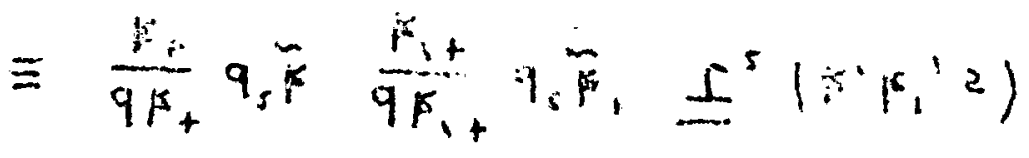

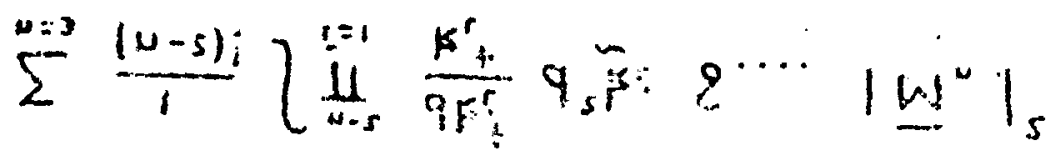

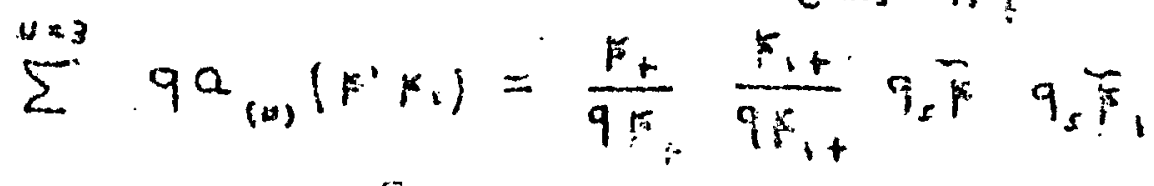

$$
\begin{aligned}
& \text { DIT fMO GLS ETUEJEg ONf }
\end{aligned}
$$

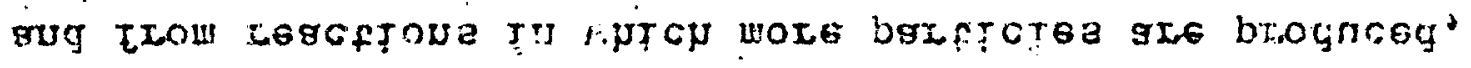

$$
\begin{aligned}
& (4 \cdot 9)^{2}
\end{aligned}
$$

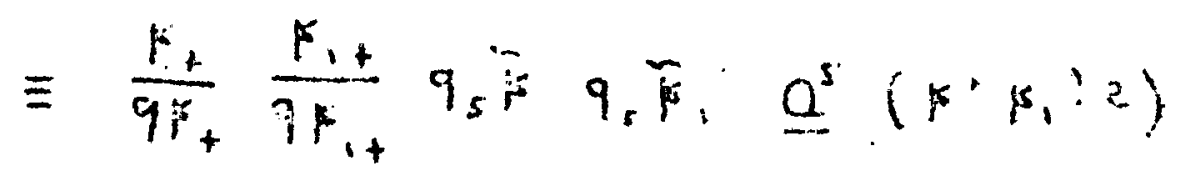

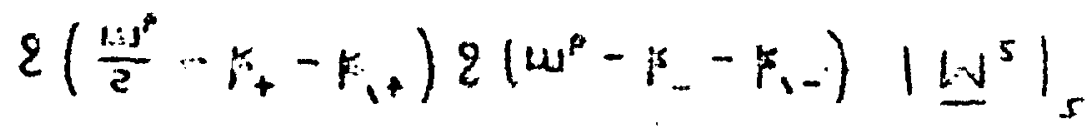

$$
\begin{aligned}
& q a_{(2)}\left(F^{\prime} F_{1}\right)=\frac{F_{+}}{q F_{+}} \frac{F_{1} t}{q \xi_{1}+} a_{s} \bar{k} q_{s} \bar{F}_{1} Q\left(\bar{F}+\bar{F}_{1}\right) \\
& \text { fMo baxffCJGa die brogncog }
\end{aligned}
$$

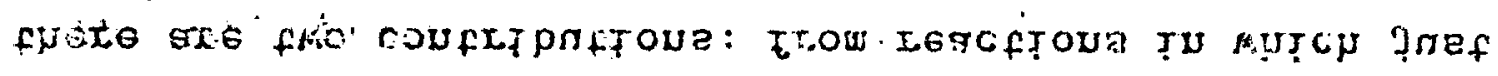

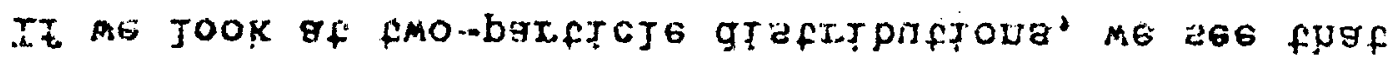

$$
\begin{aligned}
& s u^{p} F^{g}=F_{s}^{T}+w_{s}^{c}-u_{s}^{p}
\end{aligned}
$$

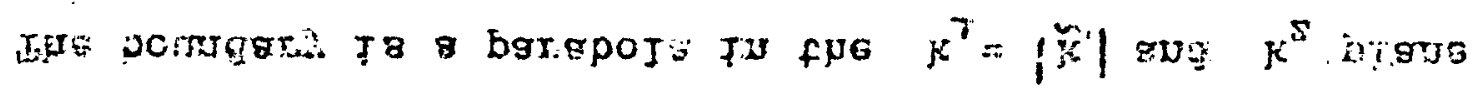


$-36-$

has a limiting form, as do the r-particle distributions

$$
\rho_{r}\left(k_{1} \ldots k_{r} ; s\right)=\bar{\sigma}_{r}\left(k_{1} \ldots k_{r} ; s\right)+\bar{\tau}_{r}\left(k_{1} \ldots k_{r} ; s\right)
$$

where the $\bar{\sigma}_{r}$ and $\bar{\tau}_{r}$ are analogously defined. The contributions to $\bar{\sigma}_{r}$ come from the surface

$$
\sum_{i=1}^{r} \bar{k}_{i}=m_{b}
$$

while the contributions to $\bar{\tau}_{r}$ come from the region

$$
\sum_{i=1}^{r} k_{i}^{-}<m_{b}
$$

Note that since

$$
\rho_{r}\left(k_{1} \ldots k_{r} ; s\right)=\delta \ldots\left|\bar{M}_{r}\right|^{2}+\sum_{n=r+1} \frac{1}{(n-r) !} \int d_{r+1} \ldots d_{n} \delta \ldots\left|\bar{M}_{n}\right|^{2}
$$

and

$$
\rho_{r+1}\left(k_{1} \ldots k_{r+1} ; s\right)=\delta \ldots\left|\bar{M}_{r+1}\right|^{2}+\sum_{n=r+2} \frac{1}{(n-r-1)^{!}} \int d_{r+2} \ldots d_{n} \delta \ldots\left|\bar{M}_{n}\right|^{2}
$$

where we have written $d_{i}$ for $d k_{i}^{+} d^{2} k_{i} / k_{i}^{+}$,

$$
\int d_{r+1} \rho_{r+1}\left(k_{1} . . k_{r+1}, s\right)=\sum_{n=r+1}(n-r) \frac{1}{(n-r) !} \int d_{r+1} \ldots d_{n} \delta \ldots\left|\bar{M}_{n}\right|^{2}
$$

Hence, as long as we stay away from the surface (4.3)

we have 
$-37-$

$$
\int \frac{d k^{+}}{k^{+}} d^{2} \underset{\sim}{k} \rho_{r+1}\left(k_{1}, \ldots k_{r}, k ; s\right)=\bar{n}, \rho_{r}\left(k_{1}, \ldots k_{r ;} ; s\right)
$$

where $\bar{\pi}_{r}$ is the average number of particles emitted In addition to the ones for which the momenta $k_{1}, k_{2}, \ldots, k_{r}$ are fixed.

Chou and Yang (70) observed that if we define

$$
\xi_{i}=\overrightarrow{k_{i}} / m_{b}
$$

then, if all the particles are identical

$$
\frac{1}{\sigma^{+0 t}} \int \frac{d k^{+}}{k^{+}} d^{2} k \xi \rho,\left(k^{+}, k ; s\right)=1
$$

Very briefly, the proof goes as follows:

We have

$$
\begin{aligned}
d \sigma_{n}\left(k_{1} \ldots k_{n}\right) & =d_{1} d_{2} \ldots d_{n} \delta \ldots\left|\bar{M}_{n}\right|^{2} \\
\rho_{1}\left(k_{1}, s\right) d_{1} & =\sum_{n=2} \frac{1}{(n-1) !} \int_{2} \ldots \int_{n} d \sigma_{n}\left(k_{1} \ldots k_{n}\right) \\
\sigma_{\text {tot }}(s) & =\sum_{n} \frac{1}{n !} \int_{1} \ldots \int_{n} d \sigma_{n}\left(k_{1} \ldots k_{n}\right)
\end{aligned}
$$

Since $\sum_{i=1}^{n} \xi_{i}=1$ we may write

$$
d \sigma_{n}\left(k_{1} \ldots k_{n}\right)=\left(\sum \xi_{i}\right) d_{1} d_{2} \ldots d_{n} \delta \ldots\left|\bar{M}_{n}\right|^{2}
$$


$-38-$

Since the particles are identical, this, as an integrand is equivalent to

$$
d \sigma_{n}\left(k_{1} \ldots k_{n}\right)=n \xi_{1} d_{1} \ldots d_{n} \delta \ldots\left|\bar{M}_{n}\right|^{2}
$$

Hence

$$
\begin{aligned}
\xi_{1} \rho_{1}\left(k_{1}, s\right) d_{1} & =\sum_{n} \frac{1}{(n-1) !} \int_{2} \ldots \int_{n} \frac{d \sigma_{n}\left(k_{1} . . k_{n}\right)}{n} \\
& =\sum_{n} \frac{1}{n !} \int_{2} \ldots \int_{n} d \sigma_{n}\left(k_{1} \ldots k_{n}\right)
\end{aligned}
$$

from which it follows that

$$
\int \xi_{1} \rho_{1}\left(k_{1} ; s\right) d_{1}=\sum_{n} \frac{1}{n !} \int_{1} \ldots \int_{n} d \sigma_{n}\left(k_{1} \ldots k_{n}\right)=\sigma_{t o t}(s)
$$

The relation generalizes to

$$
\frac{1}{\sigma_{t_{0} t}(s)} \int \frac{d k^{+}}{k^{+}} d^{2} k \sum_{x} \xi \rho_{1}^{\mathbf{z}}\left(k^{+}, k ; s\right)=1
$$

where the sum is over all species of particles $\left(p, \pi^{+}\right.$, $\left.\pi^{-}, \wedge, \ldots\right)$. The Chou -Yang sum rule implies that

$$
\int d^{2} k \frac{d k^{+}}{\left(k^{+}\right)^{2}} \rho_{1}\left(k^{+}, \underset{\sim}{k} ; s\right)<\infty
$$

unless it turns out that the total cross section grows logarithmically with $s$ as has been suggested by some (Chang and Wu (70), Chang and Man (70)), as following from 


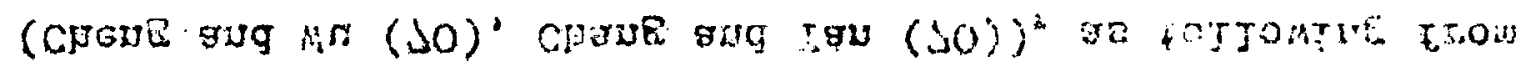

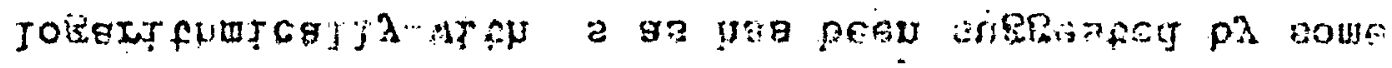

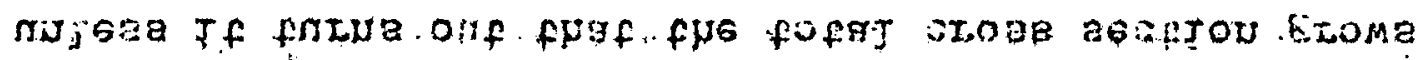

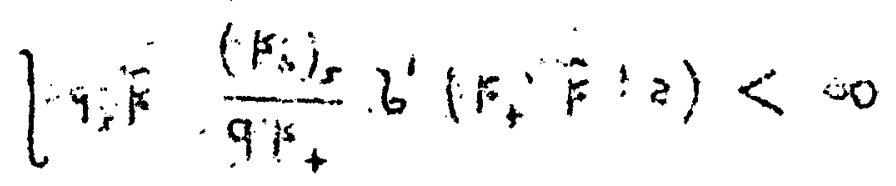

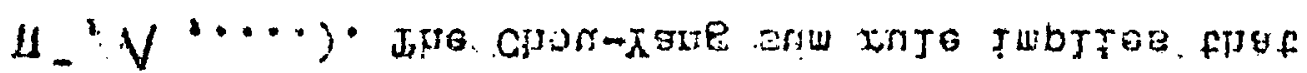

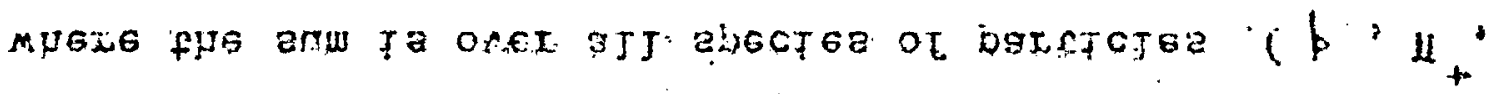

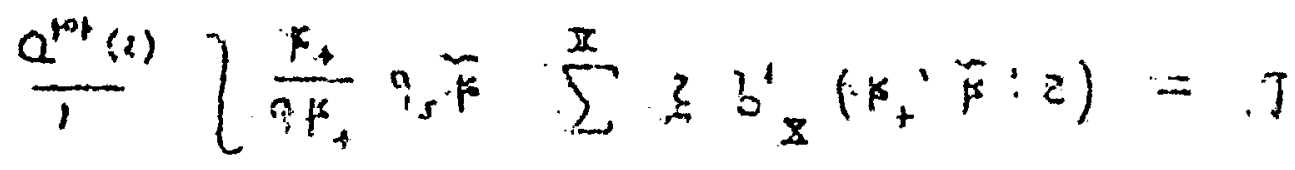

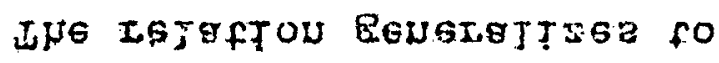

$$
\left.\left[z^{\prime} b^{\prime}\left(k^{\prime}: a\right) q^{\prime}=\sum^{m} \frac{N_{1}}{1}\right]^{\cdots}\right]^{n} q a^{n}\left(k^{\prime} \cdot k^{*}\right)=a^{+*}(z)
$$

ELow antop If tojtoma fhef

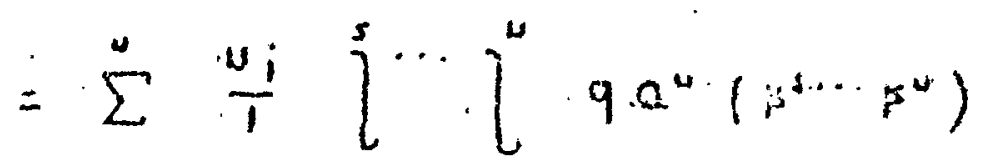

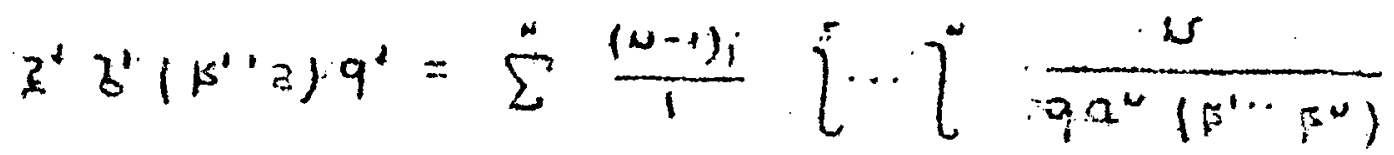

Hat5Cs

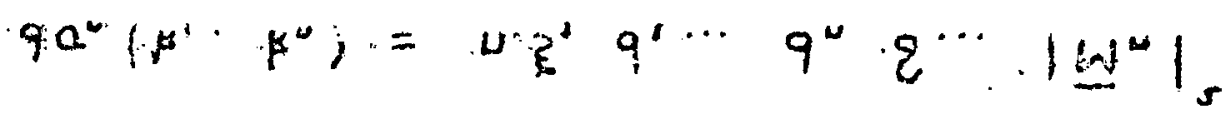

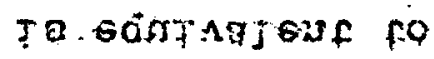

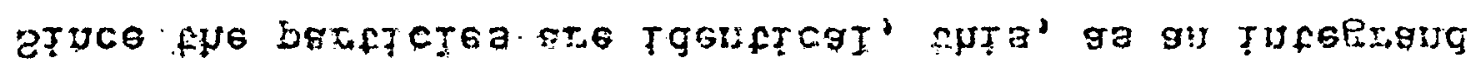


quentum field theory.

If we accept a multiplicity that grows logarithmically with $s$, then, in the limit $s \rightarrow \infty$,

$$
\int d^{2} k \frac{d k^{+}}{k^{+}} \rho_{1}\left(k^{+}, k\right) \text { diverges }
$$

The simplest way of reconciling the last two relations is to require that for large $\mathrm{k}^{+}$( of order $\mathrm{s}^{\epsilon}(\epsilon<1)$ up )

$$
\rho_{i}\left(k^{+}, \underset{\sim}{k}\right) \longrightarrow \rho_{1}(k)
$$

This implies that the one-particle distribution function becomes

$$
\rho_{1}(\underset{\sim}{k}) \frac{d k^{+}}{k^{+}} d^{2} \underset{\sim}{k}=\rho_{1}(\underset{\sim}{k}) \frac{d k_{z}}{E_{k}} d^{2} \underset{\sim}{k}
$$

When $(4.5)$ is translated into $y$ variables, we obtain

$$
\bar{n}=\frac{\int d^{2} k d y \rho_{1}(k, y)}{\sigma_{\text {tot }}}
$$

and $(4.21)$ can also be said to imply that $\rho_{1}(\underline{v}, y)$ is independent of $y$ in the region away from the neighborhood of $y=0$.

It is very enlightening to consider $\rho_{1}(\underset{\sim}{\boldsymbol{k}}, \boldsymbol{y})$ for $k$ fixed (Fig. 8 ). The range of variables is from $\mathrm{y} \approx \mathrm{y}$ at the upper end, corresponding to $\mathrm{k}^{+} \approx \mathrm{s} / \mathrm{m}_{\mathrm{b}}$, 
to $y \approx 0$ at the lower end, corresponding to $k_{\max }^{-}=m_{b}$. The fact that $\overline{\mathrm{n}}$ is proportional to $\mathrm{Y}$ implies that the area under the curve of $\rho_{1}$ vs. y must be proportional to Y. Fig. 8 shows several ways in which this can be achieved. Note that near $\mathrm{y}=0$ we are almost sure of seeing a fragment of the target $B$; similariy near $y=Y$ we are most probably seeing a fragment of the projectile A. If $\rho_{1}$ does not tend to zero in the region in the middle, then, in the common usage of the term, we do have pionization, or, to phrase it differently, we have a contribution from the multiperipheral region. In Fig. 8a. we show the distribution that might correspond to a "fireball" model: the two colliding particles are excited, and subsequently decay. As the energy goes up, they are more excited and they decay into more particles. In this way the multiplicity grows with $Y$ because the target and projectile distributions grow. Such a distribution is not limiting, but that does not mean that it is wrong! Experiments will be needed to establish limiting behavior. In Fig. $8 b$ we show the distribution that might correspond to what is depicted in Fig. 7b. If the distribution is to be limiting, it is necessary for the central plateau to grow in length as $\mathrm{Y}$ increases. There is no reason to insist that the central plateau be independent of the nature of the particles $A$ and $B$. In Fig. $8 \mathrm{c}$ we show the distribution that might be expected from the multiperipheral point of view. There is 
$-41-$

no reason for any break in the distribution; the fluctuatrons in $\rho_{1}$ in the central region must be small-scale compared to $Y$, and the distribution in the middle should, by factorization; be independent of the nature of both particles $A$ and $B$.

Most analyses of data that have a bearing on the limiting behavior of the one-particle distribution function $\rho_{1}$ have made use of a variable introduced by Feynman (69)

$$
x=\frac{2 k_{z}^{*}}{\sqrt{5}}
$$

where the asterisk is associated with the center-of mass variables. Since

$$
\frac{d k^{+}}{k^{+}}=\frac{d k_{z}}{E}=\frac{d k_{2}}{\left(m_{\perp}^{2}+k_{z}^{2}\right)^{1 / 2}}
$$

is a relation that is invariant under boost along the $z$ axis, we have

$$
\begin{aligned}
\rho_{1} & =k^{+} \frac{d^{3} \sigma}{d k^{+} d^{2} \underset{\sim}{k}}=E \frac{d^{3} \sigma}{d k_{z} d^{2} k}=E^{*} \frac{d^{3} \sigma}{d k_{z}^{*} d^{2} k} \\
& =\frac{2 E^{*}}{\sqrt{s}} \frac{d^{3} \sigma}{d x d^{2} \underline{\sim}} \\
& =\left(\frac{4 m_{\perp}^{2}}{s}+x^{2}\right)^{1 / 2} \frac{d^{3} \sigma}{d x d^{2} \underset{\sim}{k}}
\end{aligned}
$$

$(4.26)$

For $s$ large enough so that the limiting region is reached, 
the right-hand side of this equation should become independent of $s$ :

Measurements of the backward $\pi^{ \pm}$distribution in $\pi^{-}$p reactions, with the incident $\pi^{-}$energy varying between 2.15 and $6.0 \mathrm{GeV}$ were recently published by Anthony et al.(71). Fig. 9 shows a plot of these data in terms of the variable $x$ (Chen et al. (7i)), at $\mathbf{k}=0$. The values of $x$ range from -0.44 to - 0.79 , while s ranges from 5.6 to $12.2 \mathrm{GeV}^{2}$. In terms of the variables $y$ and $Y$, we have the range $3.4 \leqslant \mathrm{Y} \leqslant 4.45$ and $-1.08 \leqslant \mathrm{y} \leqslant-1.71$. Thus we are very definitely dealing with backward fragments of the target. What the figure shows is that in this range of the $x$ (or $y$ ) variable, the distribution $\rho_{1}$ is independent of $s$ for $s \gtrsim 6 \mathrm{GeV}^{2}$, in agreement with the hypothesis of limiting fragmentation. The fact that the cross section for the reaction

$$
\pi+p \rightarrow \pi^{-}+\text {anything }
$$

is somewhat smaller than that for

$$
n^{-}+p \rightarrow \pi^{+}+\text {anything }
$$

gives us some information about the fragmentation of the proton; the latter has more available channels so that the result is not surprising.

For an early test see Bali et al. (70) 
Further support for the onset of limiting behavior in $s$ comes from analysis of data from Smith et al. (69). by Chou and Yang (70). In the analysis

$$
\frac{d \sigma}{d k_{2} d^{2} k}=\frac{1}{\left(k_{2}^{2}+m_{1}^{2}\right)^{1 / 2}} p_{1}
$$

for $\pi^{-}$in $p$ - p collisions at $12.9,21.1$ and $28.4 \mathrm{GeV} / \mathrm{c}$ incident momenta is plotted with $|\underset{\sim}{\mathrm{k}}|$ taken equal to $0.2 \mathrm{GeV} / \mathrm{c}$ (for want of detailed information). Fig. 10 shows that the curves very nearly coincide, showing that for $s \gtrsim 26 \mathrm{GeV}^{2}$ the distribution appears to have reached its limiting form.

The question of the onset of limiting behavior is important, since there is evidence that in the reaction

$$
\dot{p}+p \rightarrow p+\text { anything }
$$

at $30 \mathrm{GeV} / \mathrm{c}$ incident momentum, limiting behavior has not set in. The data of Anderson et al. (67) shows that

$$
\frac{d \sigma}{d k_{z}^{*} d^{2} \underset{\sim}{k}}=\frac{1}{\left(k_{. z}^{x^{2}}+m_{\perp}^{2}\right)^{1 / 2}} \rho_{1} \approx f\left(k_{\perp}\right)
$$

depends on $|\mathbf{k}|$ alone in the region $0.8<\mathrm{k}_{z}^{*}<2.8$ $\mathrm{GeV} / \mathrm{c}$. This corresponds to $x$ values in the range $0.2-0.7$. If this is taken to be the behavior for $x$ fixed, in this range, then (4.28) would suggest 
that $\rho_{1} \simeq f\left(k_{1}\right)\left(\frac{x^{2} s}{4}+m_{1}^{2}\right)^{1 / 2}$ grows in a manner that is inconsistent with the slow growth of the multiplicity. Thus it may be that (a). limiting behavior sets in at higher energies, or (b) the curve shown in Fig.ll cuts off somewhere above $k_{z}^{*} \gtrsim 2.8 \mathrm{GeV} / \mathrm{c}$ at values of $x$ that decrease as $s$ grows. To attempt a guess at what happens we must turn to more detailed and more speculative ideas of what happens in fragmentation.

A consequence of the fragmentation idea is that the target fragment distribution should be independent of the nature of the projectile. This can be checked by looking at one-particle distributions in the backward direction $(x<0)$. Recently Ko and Lander (71) compared their data on

$$
\mathrm{K}^{+}+\mathrm{p} \rightarrow \mathrm{\pi}^{-}+\text {anything }
$$

at $11.8 \mathrm{GeV} / \mathrm{c}$ with the $25 \mathrm{GeV} / \mathrm{c}$ data of Elbert,Erwin and Walker (71) on

$$
\pi^{-}+p \longrightarrow \pi^{-}+\text {anything }
$$

Such a comparison is meaningful because we saw earlier that limiting behavior sets in at $s$ as low as $6 \mathrm{GeV}^{2}$. They found that the shape of the $\pi^{-}$distribution is the same in the two cases, as shown in Fig. 12. The absolute values of the backward cross sections differ 
by a factor of $2-3$. One would expect this ratio to be of the order of $\sigma^{\text {tot }}\left(\pi^{-} \mathrm{p}\right) / \sigma^{\text {tot }}\left(\mathrm{K}^{+} \mathrm{p}\right) \leqslant 1.5$; and the discrepancy is something of a puzzle at these energies ${ }^{+}$.

The forward distribution $(x>0)$ gives us information about the fragmentation of the projectile. We have, for large $s$ and for $k_{z}^{*} \gg m_{\pi}$ (if we look at pions anything) the following relations

$$
\begin{array}{ll}
|x|=\frac{m_{\perp}}{m_{b}} e^{-y} & x<0 \\
x=\frac{m_{\perp}}{m_{a}} e^{y-Y} & x>0
\end{array}
$$

( $A=\pi$ or $K ; B=p$ here). Ko and Lander (71) also compared their forward data with that for $\pi^{-}$in the reaction

$$
\pi^{+}+p \rightarrow \pi^{-}+\text {anything }
$$

measured by Biswas et al. at $18.5 \mathrm{GeV} / \mathrm{c}$.

+ In Regge language, fragmentation without pionization could be caused by a transmittal of a Regge pole from projectile to target. At these energies one would expect Pomeron exchange to dominate. The multiperipheral contamination should also, by definition, not depend on the projectile. 
The slope of the distribution is the same within experimental errors indicating that fragmentation of a $\pi^{+}$and a $\mathrm{K}^{+}$into $a \pi^{-}+\ldots$ is very similar. Incidentally, data from

$$
\pi^{-}+\mathrm{p} \rightarrow \pi^{-}+\text {anything }
$$

will contain a contribution from the reaction in which the projectile does not fragment (the "leading $\pi^{-}$" contribution, for $x \geq 0$ only ) and this must somehow be extracted before a meaningful comparison can be made. When this contribution is estimated, one again finds that the $\pi^{-}$slope, i.e. the parameter $b$ in

$$
\frac{d \sigma}{d x}=c e^{-b|x|}
$$

is of the order of

$$
b_{\text {forward }} \cong 7.0 \pm 0.5
$$

in agreement with the $\pi^{+}$and $\mathrm{K}^{+}$slopes. The backward slopes are all given by

$$
b_{\text {baok }} \cong 10.5 \pm 0.7
$$

within errors.

In a study of the $\pi^{-}$distribution in $12.7 \mathrm{GeV} / \mathrm{c}$ $\mathrm{K}^{+} \mathrm{p}$ interactions, stone et al. (7I) found the same 
form for the forward and backward distribution, with

$$
\begin{aligned}
& b_{\text {fwd }}=7.14 \pm 0.20 \\
& b_{\text {back }}=9.91 \pm 0.25
\end{aligned}
$$

It should be stressed that there is no reason for the.. forward and backward distributions to be the same, since, except near $x \approx 0^{+}$, they presumably refer to the fragmentation of different particles.

A curious fact was noted by Elbert et al. (71) and confirmed by stone et al.(71). If the data on $d \sigma / d p_{z}$ is plotted against $p_{z}$ in the frame in which

$$
R=\frac{\text { Imomentum of target } 1}{\text { momentum of projectile }}=1.5
$$

then it turns out that the distribution of pions in the reactions

$$
\pi^{-}+p \rightarrow \pi^{ \pm}+\text {anything }
$$

at $25 \mathrm{GeV} / \mathrm{c}$ (with the leading pion excluded) and in

The use of the variable $x$ at very high energies should be discouraged. If there is a multiperipheral component, then the particles so produced will cluster around $x \approx 0$, and information in that region may be lost. It may be that this region is uninteresting, i.e. we have independent emission and no correlations. Once that fact is established, then the variable may be useful again. 


$$
\mathrm{K}^{+}+\mathrm{p} \rightarrow \pi^{-}+\text {anything }
$$

at $12.7 \mathrm{GeV} / \mathrm{c} ;$ as well as in

$$
\pi^{+}+p \rightarrow \pi^{-}+\text {anything }
$$

at $7 \mathrm{GeV} / \mathrm{c}$, is symmetric in $\mathrm{p}_{\mathrm{z}}$ (e.g. Fig. 13). This is the sort of result that gladdens the heart of the believer in the additive quark model, and yields one more puzzle for the non-believer! +

t Stone et al. have constructed a fairly simple model that reproduces the observed asymmetry in the center of mass frame. Whether it is simpler to see the proton and the incoming meson as three quarks and quark-antiquark without interaction, respectively, is a matter of taste. 


\section{DIFFRACTIVE PROCESSES}

In two-body reactions, certain cross sections do not go to zero as 8 increases. These are called diffractive processes. Examples are

(a) all elastic scattering reactions

(b) certain resonance production reactions such as

$$
\begin{aligned}
& p+p \rightarrow p+N^{*}(1400) \\
& p+p \rightarrow p+N^{*}(1520) \\
& p+p \rightarrow p+N^{*}(1690)
\end{aligned}
$$

(see Fig.14), and

$$
\begin{aligned}
& \pi^{-}+p \rightarrow \pi^{-}+N^{*}(1400) \\
& \pi^{-}+p \rightarrow \pi^{-}+N^{*}(1520) \\
& \pi^{-}+p \rightarrow \pi^{-}+N^{*}(1690)
\end{aligned}
$$

(Anderson et al. (70)

(c) $\rho$ and $\omega$ photoproduction.

If we make use of the notion of vector dominance of photon-induced processes, we may characterize all

of these by the common property that no internal quantum numbers need be exchanged in the t-channel. We will sometimes describe this by "Pomeron exchange" without committment to any specific notion of what the Pomeron $\mathrm{is}^{+}$. Inclusion of the highest-lying (exchange-

+ Recent reports that preliminary ISR experiments on p-p scattering show no shrinkage for $s \sim 10^{3}$ imply that the Pomeron is not on a trajectory. It cannot, by unitarity, be a fixed pole in the J-plane, so that the amplitude isf(t) must be made up of some complicated set of singularities. Here an optical picture certainly seems more related to reality! 
degenerate) trajectories $\left(f^{0}, A_{2}\right)$ with intercept $\alpha(0) \cong 1 / 2$ predicts the coss section behavior

$$
\sigma(s)=A+\frac{B}{s^{1 / 2}}
$$

Gribov (67) and Morrison $(66,68)$ have suggested that in diffraction processes only spin-parity changes of $\mathrm{O}^{+}$, $1^{-}, 2^{+}, \ldots$ are permitted. Thus the target proton can be excited to $T=1 / 2$ states of spin-parity $(1 / 2)^{+}$, $(3 / 2)^{-},(5 / 2)^{+}, \ldots$ in agreement with the data of Anderson et al $(66,70)$. The same rule should apply to projectile excitation. Thus the reaction

$$
\pi^{-}+p \rightarrow A_{1}^{-}+p
$$

should be diffractive. The data of Ascoli et al. (71) is not very encouraging. It shows no sign of levellingoff up to $25 \mathrm{GeV} / \mathrm{c}$ : a fit made without theoretical prejudices yields

$$
\sigma(s) \simeq 320\left[P_{L}(\mathrm{GeV} / \mathrm{c})\right]^{-0.42} \mu b(5.2)
$$

so that if there is a constant part, this limiting behavior oes not set in until much higher energies are reached. It could be that the diffractive component is suppressed because of the parity change (Byers and Frautschi (68)).

There is, however, evidence that diffractive processes 
can take place without the final states being resonances, as first pointed out by Good and walker (60). In his review, Franzini (69) shows data on the reaction.

$$
\pi^{-}+p \rightarrow(3 \pi)^{-}+p
$$

As Fig. 15 shows, a plot of the spectrum of the threepion invariant mass, $M\left(\pi^{+} \pi^{-} \pi^{-}\right)$exhibits the following features: (a) a rapid rise at about $1 \mathrm{GeV}$, (b) a suppression of events with $M$ above $1.5-2 \mathrm{GeV}$, and (c) a broad, energy-independent peak in the region $1.0-1.5 \mathrm{GeV}$. It is true that the $\mathrm{A}_{1}$ and the $\mathrm{A}_{2}$ lie in that region, but the $A_{1}$ is quite narrow, and the $\mathrm{A}_{2}$ production should decrease like $1 / \mathrm{p}_{\mathrm{L}}$. Thus non-resonant final states are produced diffractively. Franzini estimates the constant cross section for the process to be $0.5 \mathrm{mb}$ and this is quite a bit larger than the total $A_{1}$ production cross section determined by Ascoli et al.

Further evidence for the dominance of diffractive processes in the $8-16 \mathrm{GeV}$ region comes from a longitudinal phase space analysis (Van Hove (69)) of the reaction

$$
\pi^{-}+p \rightarrow 2 \pi^{-}+\pi^{+}+p
$$

at $11 \mathrm{GeV} / \mathrm{c}$ and $16 \mathrm{GeV} / \mathrm{c}$. The analysis shows that the $\pi^{+}$and $\pi^{-}$longitudinal momenta are correlated such that if the $\pi^{+}$. goes backward in the center of mass 
system, so does one $\pi^{-}$, whereas if it goes forward, so do both $\pi^{-}$. Thus cases (c) and (d) in Fig.16 dominate, and this suggests the dominance of Pomeron exchange. (Van Hove (70))

The longitudinal phase space analysis was also applied to the reaction

$$
\pi^{+}+p \rightarrow\left\{\begin{array}{l}
\pi^{+} \pi^{\circ} p \\
\pi^{0} \pi^{+} p \\
\pi^{+} \pi^{+} n
\end{array}\right.
$$

at 8 and $16 \mathrm{GeV} / \mathrm{c}$. First the $\mathrm{T}=1 / 2(\pi N)$ production cross section was isolated from the $T=3 / 2$ cross section (the interference turned out to be small). It was found that the production of a $T=3 / 2(\pi N)$ combination decreases very rapidly with energy. The analysis shows that the cross section for $T=1 / 2$ $(\pi N)$ is constant in the region of phase space corresponding to the ( $\pi N$ ) moving backward in the c.m.s. A rough estimate yields $0.45 \pm 0.04 \mathrm{mb}$ for the constant part of the cross section (ABBCC Collaboration (71)).

Finally one may, with Chou and Yang (70), find support for diffractive processes in the observation made with cosmic rays, that the ratio $\mu^{+} / \mu^{-}$at sea level remains at $\sim 1.25$ up to $100 \mathrm{GeV}$ ( $\mathrm{Pal}$ and Peters (64)). If at high energies the dominant mechanisms were high multiplicity mechanisms, then on statistical grounds the ratio of $\pi^{+} / \pi^{-}$and hence $\mu^{+} / \mu^{-}$would approach one. 
The imbalance can only be understood if some low multiplicity cross sections remain constant up to these energies.' Much needs to be done to clarify diffraction dissociation:

(a) Do the Gribov-Morrison rules really hold? So far the evidence for them comes from $N^{*}$ production in the data of Anderson et al. and from the spin-parity analysis of $\pi^{-} \rightarrow(\rho \pi)^{-}$and $K \rightarrow\left(K^{*} \pi\right)$ in nuclei, which shows alignments corresponding to $1^{+}$(Good (1970)).

(b) Can both incoming particles be diffractively excited? So far the evidence is that only one of them is so excited, but this may be for kinematic reasons: in the reaction

$$
\mathrm{A}+\mathrm{B} \rightarrow \mathrm{C}+\mathrm{D}
$$

the minimum transfer is

$$
t_{\min }=-\frac{\left(M_{c}^{2}-M_{A}^{2}\right)\left(M_{D}^{2}-M_{B}^{2}\right)}{S}
$$

and $a t$-dependence of the form $e^{\text {at }}$ could suppress the cross section at energies studied so far ${ }^{+}$. Experiments at higher energies will provide the answer. (c) Do more massive and higher multiplicity clusters get produced at higher energies? There is some evidence

t The positive excess is presumably due to the fact that the primaries are protons. ${ }^{+{ }^{+}}$This is not the explanation if the dependence is of the form $e^{-a\left|t-t_{\text {min }}\right|}$. 
from

$$
\pi^{-}+\text {Nucleus } \rightarrow(5 \pi)^{-}+\text {Nucleus }
$$

at $16 \mathrm{GeV} / \mathrm{c}$ (Huson et al. (68)) that diffractive production of $5 \pi$ states exists, appears to peak at around $2 \mathrm{GeV}$ and has a cross section of about $15 \mu \mathrm{b}$ per nucleon. The cross section seems to grow with energy (Franzini (69)). Since the diffractive cross sections remain constant, they must fall as the multiplicity increases, but they may fall as slowly as $1 / n^{2}$ and still form a convergent series. This would have to be the situation for a pure fragmentation model with no pionization and growing multiplicity, or in language introduced in Sec.III, a two-cluster multiperipheral model.

The multiplicity will be connected to the mass of the diffractively produced cluster. Let us examine the kinematical and dynamical constraints on the mass. Fig. 17 shows the variables. Momentum conservation yields

$$
p_{a}^{ \pm}-\sum_{i=1}^{n-1} k_{i}^{ \pm}=-Q^{ \pm}=k^{ \pm}-p_{b}^{ \pm}
$$

and in the laboratory frame we have

$$
p_{a}^{+}=\frac{s}{m_{b}} ; \quad p_{a}^{-}=\frac{m_{a}^{2} m_{b}}{s} ; p_{b}^{ \pm}=m_{b}
$$

If we define $x_{i}$ by 
$-55-$

$$
k_{i}^{+}=\frac{s}{m_{b}} x_{i}
$$

then

$$
\frac{s}{m_{b}}\left(1-\sum_{i=1}^{n-1} x_{i}\right)=k^{+}-m_{b}=-Q^{+}
$$

For $t=Q^{+} Q^{-}-\underline{Q}^{2}$ to be bounded, and for the single fragment of the target particle $b$ to have a momentum that does not grow with $s$, we must have

$$
1-\sum_{i=1}^{n-1} \ddot{x}_{i}=O\left(\frac{1}{5}\right)
$$

Also, since

$$
\frac{m_{a}^{2} m_{b}}{s}-\frac{m_{b}}{s} \sum_{i=1}^{n-1} \frac{m_{1 i}^{2}}{x_{i}}=k^{-}-m_{b}=-Q^{-}
$$

can only be large if $x_{i}$ for some particles $\sim s^{-1-\epsilon}$ which is certainly counter to the fragmentation spirit, we also have $Q^{-} \sim O(1 / s)$, so that $t=-Q^{2}$, and

$$
\begin{aligned}
M^{2}=\left(\sum_{i} k_{i}\right)^{2} & =\left(\sum_{i} k_{i}^{+}\right)\left(\sum k_{i}^{-}\right)-\left(\sum k_{i}\right)^{2} \\
& =\left(\frac{s}{m_{b}} \sum_{i} x_{i}\right)\left(\frac{m_{b}}{s} \sum_{i} \frac{m_{\perp i}^{2}}{x_{i}}\right)+t \\
& \approx \sum \frac{m_{\perp i}^{2}}{x_{i}}+t
\end{aligned}
$$

This can only be large if some of the $x_{i}$ become very small. If the $k_{i}$ really only describe fragments of the projectile, then the $x_{i}$ cannot be as small as $O\left(s^{1 / 2}\right)$, and 
$-56-$

$M^{2}$ cannot grow rapidly with $\mathrm{s}$.

Incidentally, this argument restricts the mass more

than coherence arguments do. The center of mass momenta

of the projectile $a$ and the diffractively produced

state (of mass $M$ ) are respectively

$$
\begin{aligned}
& p_{a}^{2}=\left(\frac{s+m_{a}^{2}-m_{b}^{2}}{2 \sqrt{5}}\right)^{2}-m_{a}^{2} \\
& p_{M}^{2}=\left(\frac{s+M^{2}-\mu^{2}}{2 \sqrt{s}}\right)^{2}-M^{2}
\end{aligned}
$$

where $k^{2}=\mu^{2}$ : The coherence condition reads

$$
\left|P_{M}^{*}-P_{a}^{*}\right| R<1
$$

where $R$. is the radius of the interaction region, assumed constant. The condition thus takes the form

$$
\begin{aligned}
\frac{1}{R}>\frac{p_{a}^{2}-p_{M}^{2}}{p_{a}^{2}+p_{M}^{*}} & \approx \frac{1}{\sqrt{s}}\left(\frac{\left(s+m_{a}^{2}-m_{b}^{2}\right)^{2}}{4 s}-\frac{\left(s+M^{2}-\mu^{2}\right)^{2}}{4 s}+M^{2}-m_{a}^{2}\right) \\
& \approx \frac{1}{2 \sqrt{5}}\left(M^{2}+\mu^{2}-m_{a}^{2}-m_{b}^{2}\right) \\
& \approx \frac{M^{2}}{2 \sqrt{5}}
\end{aligned}
$$

ie.

$$
M^{2}<\frac{2 \sqrt{5}}{R}
$$

If we allow the fragment momentum distribution to have a long tail, which, according to the discussion of 
of Sec.IV is necessary to keep distributions limiting while the multiplicity grows as log $s$, but which also is indistinguishable from a multiperipheral distribution, then $M^{2}$ can grow like $\sqrt{\mathrm{s}}$, for example. One way in which a two-cluster model could explain the multiplicity, is to assume that (a) $M^{2} \leq s^{\alpha}$ $(\alpha \leq 1 / 2)$ tand (b) that the "cluster" is an object that decays into pions, with an average multiplicity proportional to the mass. This is necessary for the boundedness of the transverse momentum in the "cluster" rest frame, and it is also a characteristic of statistical models. For example, the phase space expression for the decay of an object of mass $M$ into $n$ massless pions,

$$
\Phi_{n}=\left(\frac{M^{2}}{16 \pi^{2}}\right)^{n-2} \frac{8 \pi}{(n-1) !(n-2) !}
$$

peaks at $n=M / 4 \pi$. A model embodying this point of view has been constructed by Hwa $(71)^{++}$.

It w1Il not be easy to distinguish between a purely diffractive picture, favored by BCYY and Hwa, and a picture in which low multiplicity events are

This is not meant to imply that all clusters are so massive, but merely that this is a realizable upper limit for the mass of the cluster. ${ }^{++}$A similar model, with a different objective, was also discussed by Rajaraman (70). 
diffraction-dominated, and high multiplicity events are multiperipheral, as proposed by Wilson (70), who also suggested the following test: consider $p-p$ interactions at fixed, high $s$, and study $\sigma_{n c}(s)$ for the production of n charged secondaries. According to Wilson's view there should be a dip in $\sigma_{n c}(s)$ vs. $n$ between small. $\mathrm{n}$ (diffraction) and the region $\mathrm{n} \sim \log \mathrm{s}$. Unfortunately $\log s$ is not that large, and fluctuations of order $(\log s)^{1 / 2}$ could wash out the dip, especially if both the target and the projectile undergo diffraction dissociation. Another test might be to study pions emitted at $90^{\circ}$ in the center of mass system. These should come from the multiperipheral component, and their distributions should be independent of the incident particles.

A possible way to study the diffraction dissociation at one vertex (as a function of $M^{2}$ ) is to work in the kinematic region corresponding to the graph of Fig.17, with the target unchanged in the collision. Following the discussion in Sec.IV we see that we must work near the kinematic boundary given by eq. (4.7) i.e. at

$$
k^{-}=m_{b}\left(1-O\left(m^{2} / s\right)\right)
$$

where all the $k_{i}^{-}$are of order $\mathrm{m}^{2} / \mathrm{s}$, i.e. most probably fragments of the projectile. For fixed momentum transfer,

For two-body reactions such cross sections are very small, because they involve very large momentum transfers. This need not be the case when there is a multiperipheral component. 
$-59-$

$$
t=\left(p_{b}-k\right)^{2}=2 m_{b}^{2}-2 m_{b} k_{0}
$$

we may approach the kinematic boundary by decreasing $k_{z}$. The allowed region is defined by

$$
k_{0}-k_{2} \leq m_{b}
$$

i.e.

$$
\begin{aligned}
k_{2} \geqslant k_{0}-m_{b} & =\frac{2 m_{b}^{2}-t}{2 m_{b}}-m_{b} \\
& =-\frac{t}{2 m_{b}} \equiv k_{z}^{b d r y}
\end{aligned}
$$

Note that $(5.13)$ implies that $k_{z}^{\text {dry }}=k_{\perp}^{2} / 2 m_{b}$ ie. $t=-k_{\perp}^{2}$. Now the distance in $k_{z}$ from the boundary is related to the mass of the cluster at the projectile vertex. We have

$$
\begin{aligned}
M^{2}=\left(p_{a}+p_{b}-k\right)^{2} & \cong s-k^{+} m_{b}-k^{-} \frac{s}{m_{b}}+m_{b}^{2} \\
& \cong \frac{s}{m_{b}}\left(m_{b}-k_{b}+k_{z}\right) \\
& =\frac{s}{m_{b}}\left(k_{z}-k_{z}^{\text {dry }}\right)
\end{aligned}
$$

Hence with $t$ fixed and $s$ large and fixed, it is possible by varying

$$
\delta=\left(k_{z}-k_{z}^{b d r y}\right)
$$

to vary $M^{2}$. 
More concretely, the amplitude corresponding to the graph on Fig.l7 is given by

$$
M=\gamma\left(p_{a}, Q_{i} k_{1}, \ldots k_{n-1}\right) \beta_{b}(t)\left(\frac{s}{M^{2}}\right)^{\alpha_{p}(t)}
$$

Here $\gamma\left(p_{a}, q ; k_{1} \ldots k_{n-1}\right)$ is a generalized vertex function, $\beta_{b}(t)$ is the more familiar particle-pomeron coupling, and the last factor is the standard Regge propagator. Generally such a factor is written as $\left(s / s_{0}\right)^{\alpha}(t)$ because the masses of the incoming and outgoing states are fixed, but here the variation of one of the masses $\left(M^{2}\right)$ is important. The dependence comes from the fact that the $s$ dependence really comes from $P_{\alpha_{p}}\left(-z_{t}\right)$. for $\left.\left|z_{t}\right| \gg\right\rangle$, where $z_{t}$ is the cosine of the scattering angle in the $t$-channel, and

$$
z_{t}=\left(\frac{t}{t-4 m_{b}^{2}}\right)^{1 / 2} \frac{2 s+t-M^{2}-m_{a}^{2}-2 m_{b}^{2}}{\left[\left(t-M^{2}-m_{a}^{2}\right)^{2}-4 M^{2} m_{a}^{2}\right]^{1 / 2}}
$$

If the target remains a single particle, but changes its mass to $\mu$, then (cf.eq. (2.21))

$Z_{t}=\frac{t\left(2 s+t-M^{2}-m_{a}^{2}-m_{b}^{2}-\mu^{2}\right)+\left(M^{2}-m_{a}^{2}\right)\left(\mu^{2}-m_{b}^{2}\right)}{\left[\left(t-\left(\mu-m_{b}\right)^{2}\right)\left(t-\left(\mu+m_{b}\right)^{2}\right)\left(t-\left(M-m_{a}\right)^{2}\right)\left(t-\left(M+m_{a}\right)^{2}\right]^{1 / 2}(5.20)\right.}$

${ }^{+}$Reggeism has its origin in the $t$-channel partial wave expansion. Our expression does not take into account helicity. 
For $s \gg M^{2} \gg m_{a}^{2}, m_{b}^{2}, t$ the relevant dependerice is $s / M^{2}$. Another way of obtaining this dependence is to require that $(5.18)$ go over smoothly into what one obtains in the multi-Regge region. There we have, with $s_{12}=\left(q+k_{n-1}\right)^{2}$ and $s_{2 n}=\left(k_{1}+k_{2}+. . k_{n-1}\right)^{2}=m^{2}$ the result that

$$
\left(s_{12}\right)^{\alpha_{p}(t)}=\left(\frac{s_{12} s_{2 n}}{s_{2 n}}\right)^{\alpha_{p}(t)}=\left(\frac{S}{M^{2}}\right)^{\alpha_{p}(t)}
$$

Not surprisingly, the same factor emerges in dual models ( see e.g. Chang et al.(70)).

The cross section is given by

$$
\begin{gathered}
d \sigma=\frac{(2 \pi)^{4}}{4 F_{a b}} \frac{d^{3} k}{2 k_{0}} \frac{d^{3} k_{1}}{2 k_{10}} \cdots \frac{d^{3} k_{n-1}}{2 k_{n-1,0}} \delta\left(k_{1}+\cdots+k_{n-1}+q-p_{a}-p_{b}\right) \\
\quad\left|\gamma\left(p_{a}, Q_{;} k_{1} \ldots k_{n-1}\right)\right|^{2}\left|\beta_{b}(t)\right|^{2}\left(s / M^{2}\right)^{2 \alpha_{p}(t)}
\end{gathered}
$$

Hence

$$
\text { 2k. } \begin{aligned}
\frac{d \sigma}{d^{3} k}= & \frac{(2 \pi)^{4}}{4 F_{a b}}\left|\beta_{b}(t)\right|^{2}\left(s / M^{2}\right)^{2 \alpha_{p}(t)} \\
& \sum_{n=2} \int \prod_{i=1}^{n-1} \frac{d^{3} k_{i}}{2 k_{i b}} \delta\left(\sum_{i=1}^{n-1} k_{i}-p_{a}-Q\right)\left|\gamma\left(p_{a}, Q_{i} k_{1} \ldots\right)\right|^{2}
\end{aligned}
$$

We may now define a "cross section" for the process

$$
\text { "Pomeron" }+\mathrm{A} \longrightarrow \text { anything }
$$

and this is given by 
$-62-$

$$
\dot{\sigma}\left(M^{2}, t\right)=\frac{(2 n)^{4}}{4 F_{a r}} \sum_{n=2} \int_{i=1}^{n-1} \frac{d^{3} k_{i}}{2 k_{i 0}} \delta\left(p_{a}+Q-\sum k_{i}\right)|\gamma|^{2}
$$

With

$$
F_{a p}=\left[\left(p_{a} \cdot Q\right)^{2}-m_{a}^{2} t\right]^{1 / 2} \approx \frac{M^{2}}{2}
$$

wo finally get ${ }^{+}$

$2 k_{0} \frac{d \sigma}{d^{3} k} \approx\left|\beta_{b}(t)\right|^{2} \sigma\left(M^{2}, t\right)\left(\frac{5}{M^{2}}\right)^{2 \alpha_{p}(t)-1}$

$(5.24)$

Note that we require that $m^{2} / s=\delta / m_{p} \ll 1$. All that we know about $\sigma\left(M^{2}, t\right)$ is that when the incoming particle is a pion, it has a bump coming from three pion states in the region $M=1.0-1.5 \mathrm{GeV}$, and more detailed structure shows up at values of $M$ corresponding to baryon resonanes of $\mathbf{T}=1 / 2$ when the incoming particle is a proton. If the Pomeron acted like a particle we might expect that

$$
\sigma\left(M^{2}, t\right) \sim A(t)+\frac{B(t)}{\left(M^{2}\right)^{1 / 2}}
$$

with the second term coming from the exchange of the

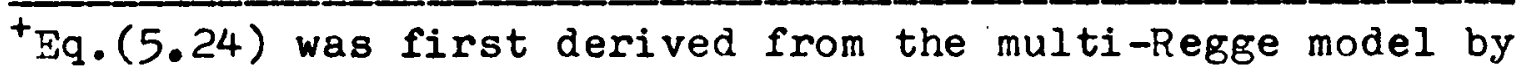
Chew, G.F. and Pignotti, A., 1968 Summer Study, Vol.3, N.A.I. 1968. It also appears in Caneschi and Pignott1 (69), Silverman and Tan (70), DeTer et al.(71), Peccei and Pignotti (71) and Feynman (69). For a really quantitative comparison, $\beta_{b}(t)$ should be redetermined from two-body reactions using $P_{\alpha}\left(-z_{t}\right)$ rather than the conventional $\left(s / s_{0}\right)^{\alpha}$. 
degenerate $\rho, P^{\prime}, A_{2}, \ldots$ trajectories. There is a difficulty with this, however. Making use of the fact that we may write

$2 k_{0} \frac{d \sigma}{d^{2} k d k_{z}}=\frac{2}{\pi} k^{-} \frac{d \sigma}{d k_{\perp}^{2} d k^{-}}=\frac{2 s}{\pi} \frac{d \sigma}{d t d M^{2}}$

we get, with a flat Pomeron, $\alpha_{p}(t)=\alpha$,

$$
\begin{aligned}
& \frac{d \sigma}{d t}=\frac{\pi}{2 s} \int_{m^{2}}^{m \sqrt{s}} d M^{2}\left|\beta_{p}(t)\right|^{2} s^{2 \alpha-1}\left(M^{2}\right)^{1-2 \alpha} \sigma\left(M^{2}, t\right) \\
& \cong \frac{\pi}{2} s^{2 \alpha-2} A(t)\left|\beta_{p}(t)\right|^{2} \frac{\left(m^{2} s\right)^{1-\alpha}}{2(1-\alpha)} \quad \alpha<1 \\
& \simeq \frac{\pi}{2} A(t)\left|\beta_{p}(t)\right|^{2} \frac{1}{2} \log s \quad \alpha=1
\end{aligned}
$$

Thus if $\alpha=1$, do/dt and aiso $\sigma$ grow like log s. This is not inconsistent with general principles (the Froissart (61) limit allows (log $s)^{2}$ growth), but there is no evidence for such growth at this time, and from a geometrical point of view double diffraction is difficult to understand: One may get around this by modffying (5.25) or by requiring that $\alpha<1$. It is the latter point of view that has excited much interest among theorists working on multiperipheral models ( Chew and Pignotti ( 68 ), Abarbanel et al. (71)). The total cross section data show

\footnotetext{
+ This is especially true if the Pomeron is indeed flat,
} as suggested by preliminary experiments at very large $s$. 
that $\alpha$ must be very close to 1 , and this makes the proposal somewhat artificial ${ }^{+}$. The difficulty, incidentally, persists if the Pomeron lies on a trajectory (Finkeistein and Kajantie (68)). The same kind of difficulties arise in a three cluster model (Fig.6) when two Pomerons are exchanged, since there is an additional power of log $\mathrm{s}$ for each Fomeron. It would be interesting to check whether experiments bear out the suppression of double Pomeron exchange.

In the $25 \mathrm{GeV} / \mathrm{c}$ region, the analysis of Lipes et al (69) shows that there is no sign of double pomeron exchange in the reaction

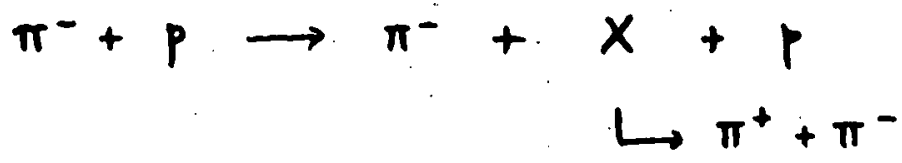

Wilson (70) suggests the following experiment to test whether the mechanism is present at higher energies. Consider the reaction

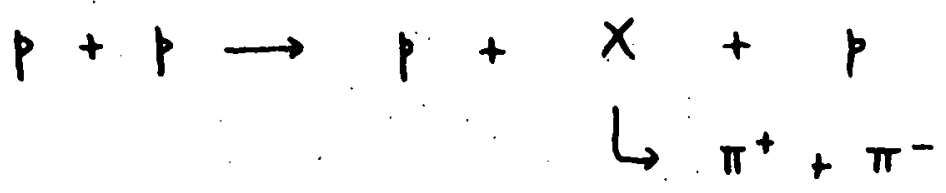

with nion pairs very slow in the center of mass frame of the incident particles. In the $25 \mathrm{GeV} / \mathrm{c}$ region, with both $s_{X p}$ large, the matrix element is presumably described by a Pomeron and a meson trajectory $(\rho, \ldots)$ as in the Iipes et al. reaction. The $x$ will therefore

+ This is a subjective statement. Chew and collaborators associate the deviation from. I with the smallness of a triple Fomeron coupling ( see p. 70). 
consist of a $\rho$ some of the time, and the cross section, as a function of $M_{X}^{2}$ will peak in the $\rho$ region. If the double Pomeron mechanism exists, it will take over at some higher energy. With two Pomerons; the $\mathrm{X}$ must have $T=0$, i.e. it cannot be a $\rho$. One would therefore expect to see a broadening of the $\rho$ peak, though there is no way of estimating at what energies this woula happen. 
VI. REGGEISM FOR INCLUSIVE REACTIONS

In this section we continue our discussion of inclusive processes, i.e. reactions of the type

$$
\mathrm{A}+\mathrm{B} \longrightarrow \mathrm{C}+\text { anything }
$$

and begin with a generalization of the configuration shown in fig.17, to the situation that $C \neq B$. We are stili in the kinematic region ${ }^{+}$

$$
k^{-}=m_{b}\left(1-O\left(m^{2} / s\right)\right)
$$

If $C \neq B$, trajectories other than the Pomeron will contribute to the exchange, and eq.(5.18) must be generalized to

$M=\sum_{i} \gamma_{i}\left(p_{a}, Q_{i} k_{1} \ldots k_{n-1}\right) \beta_{b c}^{i}(t)\left(\frac{s}{M^{2}}\right)^{\alpha_{i}(t)}$

where the subscript refers to the exchanged trajectories, and $\gamma_{i}, \beta_{b c}^{i}$ are the couplings of the trajectories at the two vertices. Algebraic manipulations identical to those following eq. (5.21) lead to the inclusive cross section

+ In this section, as at the end of Sec.V we ignore the experimental problems associated with obtaining the resolutions necessary to explore the region in the vicinity of the kinematic boundary. 
$-67-$

$2 k_{0} \frac{d \sigma}{d^{3} k} \cong \sum_{i j} \beta_{b c}^{i}(t) \beta_{b c}^{j}(t)^{*} \sigma_{i j}\left(M^{2}, t\right)\left(\frac{s}{M^{2}}\right)^{\alpha_{i}(t)+\alpha_{j}(t)-1}$

where

$$
\begin{aligned}
\sigma_{i j}\left(M^{2}, t\right) \approx & \frac{(2 \pi)^{4}}{M^{2}} A_{i j}\left(M^{2}, t\right) \\
\approx & \frac{(2 \pi)^{4}}{2 M^{2}} \sum_{n} \int \prod_{l=1}^{n-1} \frac{d^{3} k_{l}}{2 k_{0 l}} \delta\left(p_{a}+Q-\sum k_{l}\right) \\
& \left.\times \gamma_{i}\left(p_{a}, Q_{i} k_{1} . . k_{u-1}\right) \gamma_{j}^{*}\left(p_{a}, Q_{i} k_{1} . . k_{n-1}\right)\right)_{(6.4)}
\end{aligned}
$$

is the "cross section" for the reaction

$$
A+(\text { Reggeon })_{i} \longrightarrow A+(\text { Reggeon })_{j}
$$

in the forward direction. Wang and Wang (71) made the intriguing suggestion that duality considerations be applied to $A_{i j}$. If duality is applicable, one would expect two contributions to $A_{i j}$ : one, in which the trajectories "scatter" off particle A by Pomeron exchange, the other, in which they exchange a series of trajecttories k. According to duality, the former describes the background, and the latter can be replaced by a sum of resonance terms (Freud (68); Harar (68)), ie. resonances in the variable $\mathrm{M}^{2}$. The chain of reasoning is illustrated in Fig. 18. We thus write

$$
A_{i j}\left(M^{2}, t\right)=\eta_{i j p}(t) \beta_{A A}^{p}(\theta)\left(M^{2}\right)^{\alpha_{p}(0)}+A_{i j}^{\text {Res }}
$$


There are now two classes of reactions to be considered: in the first the internal quantum numbers of particles $B$ and C. are identical, so that the Pomeron can couple to them; in the second $B \neq C$ in the above sense, so that " $i$ " will not include the Fomeron. Examples of the first class of reactions are

$$
p+p \rightarrow p+x
$$

and

$$
\pi^{-}+p \rightarrow p+x
$$

Actually the reaction

$$
\pi^{-}+p \rightarrow \pi^{-}+x
$$

also belongs to this class, provided the $\pi^{-}$is a leading particle, i.e. it carries off most of the momentum. This corresponds to reversing "projectile" and "target" in Fig.17. Since the inclusive cross section is invariant

$$
2 k_{0} \frac{d \sigma}{d^{3} k}=\frac{25}{\pi} \quad \frac{d \sigma}{d t d M^{2}}
$$

and so is the condition for working near the kinematic boundary, when written in the form (cf. (5.16))

$$
\frac{M^{2}}{S}<<1
$$

the last reaction can be analyzed as is, provided the cross section is plotted as a function of $t$ and $M^{2}$. 
$-69-$

For these reactions it is enough to let "i" range over the Pomeron $(P)$ and exchange-degenerate Vector-Tensor meson trajectories $(M)$, with $\alpha_{M}(0)=1 / 2$. Then (6.3) reads

$$
\begin{aligned}
& \frac{4 s^{2}}{(2 \pi)^{5}} \frac{d \sigma}{d t d M^{2}} \\
& =\left|\beta_{b c}^{p}(t)\right|^{2}\left(\frac{s}{M^{2}}\right)^{2 \alpha_{p}(t)}\left(\eta_{p p p}(t) \beta_{a a}^{p}(0)\left(M^{2}\right)^{\alpha_{p}(0)}+A_{p p}^{R e s}\right) \\
& +\operatorname{Re}\left(\beta_{b c}^{p}(t) \beta_{b c}^{M}(t)^{*}\right)\left(\frac{s}{M^{2}}\right)^{\alpha_{p}(t)+\alpha_{M}(t)} \\
& \quad \times\left(\eta_{p P M}(t) \beta_{a a}^{p}(0)\left(M^{2}\right)^{\alpha_{p}(0)}+A_{P M}^{R e s}\right) \\
& +\left|\beta_{b c}^{M}(t)\right|^{2}\left(\frac{s}{M^{2}}\right)^{2 \alpha_{M}(t)}\left(\eta_{P M M}(t) \beta_{a a}^{p}(0)\left(M^{2}\right)^{\alpha_{p}(0)}+A_{M M}^{\text {Res }}\right)
\end{aligned}
$$

after the appropriately written form of (6.5) is substituted. For reactions of the second class, only the third term remains, if we restrict ourselves to the dominant meson exchange. The modification required if baryon exchange is required, or if there are competing trajectories, is trivial to carry out. For example in

$$
\pi^{-}+p \rightarrow p+X
$$

with $p$ in the backward direction we would expect only the last term with the $\Delta$ (instead of the $M$ ) trajectory contributing ( and $t$ replaced by $u$ ). 
Vang and Wang $^{+}(71)$ have observed the following: (a) If the triple-Fomeron coupling is negligible, as suggested by recent studies of the multiperipheral bootstrap models (e.g. Chew et al. (70)), so that

$$
\eta_{P P p}(t) \approx 0
$$

then the resonant contribution, $A_{P P}^{R e s}\left(M^{2}, t\right)$, if it is nonvanishing, grows relative to the background (coming from the second term) as $s$ increases. The relative importance grows as $\left(s / M^{2}\right)^{\alpha_{p}-\alpha_{M}} \sim s^{1 / 2}$. Also the

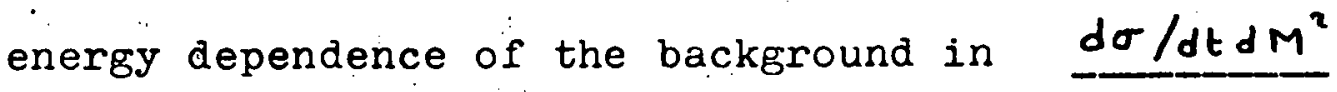
should be $\sim s^{\alpha_{M}-1}$. Figures 19 and 20 show the fall-off of the background with growing $s$, as seen in the data of Anderson et al. (66), (69), (70). An estimage of the rate of fall-off of the background is consistent with the prediction. (b) When there is no pomeron exchange, there should be no energy dependence of the background relative to the resonant contribution. Fig. 21 shows data on

$$
\pi^{-}+p \rightarrow p+X \text { ( } p \text { backward) from Anderson et al. (69) }
$$

which supports the prediction.

The beautiful analysis of Wang and Wang give us much reasion to be encouraged about the extension of ideas developed in two-body reactions to inclusive reactions. So far, however, we have limited ourselves to the kinematical region that most closely simulates two-body reaction conditions, i.e. the case where the 
target fragments into just one particle. There are two other situations that need to be discussed: (i) the case where the particle under consideration is just one of several fragments of the target ${ }^{+}$, and (ii) the case where the particle is a pionization product. The first of these corresponds to a nonvanishing $\mathrm{k}^{-}$ constrained by

$$
\mathrm{k}^{-}<\mathrm{m}_{\mathrm{b}}
$$

It should be stressed that $\mathrm{k}^{-}$cannot be too small. If it is $O\left(s^{-\not / 2}\right)$ the particle is a pionization product; if it is $O\left(s^{-1}\right)$ the particle is a fragment of the projectile. Here

$$
\begin{aligned}
M^{2}=\left(p_{a}+p_{b}-k\right)^{2} & \simeq s-k^{-} p_{a}-k^{+} p_{b}-\cdots \\
& \simeq s-k^{-} s / m_{b} \\
& \simeq s\left(1-k^{-} / m_{b}\right)
\end{aligned}
$$

i.e. $\mathrm{M}^{2} / \mathrm{s}$ is smaller than 1 but not infinitesimally small. The second case may be characterized by the fact that the particle momentum is finite (i.e. not of order $s$ or $s^{1 / 2}$ ) in the center of mass frame. Equivalently we may say that $k$ is relativistic with respect to

F In our discussion we have concentrated on the fragmentation of the target. To discuss the fragmentation of the projectile it is simplest to make a Lorentz transformation to the rest frame of particle $A$. There $p_{a}=\left(m_{a}, 0, m_{a}\right), p_{b}=\left(m_{b}^{2} m_{a} / s, 0, s / m_{a}\right)$ the kinematic boundary is $k^{+}=m_{a}$ in terms of the projectile system variables, etc. 
both $\mathrm{p}_{a}$ and $\mathrm{p}_{\mathrm{b}}$. In both cases the particle under consideration is not sufficiently distinguished to allow us to write down some sort of matrix element that would be the appropriate counterpart of (5.18), unless we assume some sort of multiperipheral model. If we consider the matrix element that corresponds to fig.22, the inclusive cross section is given by

$$
\begin{aligned}
& 2 k_{0} \frac{d \sigma}{d^{3} k}=\frac{(2 \pi)^{4}}{4 F_{a b}} \sum_{n} \int_{i=1}^{n} \frac{d^{3} k_{i}}{2 k_{0 i}} \delta\left(k_{1}+\cdots+k_{n}+k-p_{a}-p_{b}\right) \\
&\left|\gamma_{a}\left(p_{a}, Q_{1} ; k_{1} \ldots k_{r}\right)\right|^{2} \\
&\left|\gamma_{b}\left(p_{b}, Q_{2} ; k_{r+1} \ldots k_{n}\right)\right|^{2} \\
&\left|\gamma\left(Q_{1}, Q_{2}\right)\right|^{2}\left(z_{t_{1}}\right)^{2 \alpha_{1}\left(t_{1}\right)}\left(z_{t_{2}}\right)^{2 \alpha_{2}\left(t_{2}\right)}
\end{aligned}
$$

Here the $\boldsymbol{\gamma}$ 's are the couplings of the Reggeons at the three vertices, and the last two factors are the variables that for large cluster masses approach $s_{L} / M_{L}^{2}$ and $s_{R} / M_{R}^{2}$ respectively. In general

$$
\begin{aligned}
z_{t_{1}}= & \left\{t_{1}\left[2 s_{L}+t_{1}-M_{L}^{2}-m_{a}^{2}-\mu^{2}-t_{2}\right]+\left(M_{L}^{2}-m_{a}^{2}\right)\left(\mu^{2}-t_{2}\right)\right\} \\
& \left\{\left(t_{1}-t_{2}-\mu^{2}\right)^{2}-4 \mu^{2} t_{2}\right\}^{-1 / 2}\left\{\left(t_{1}-M_{L}^{2}-m_{a}^{2}\right)^{2}-4 M_{L}^{2} m_{a}^{2}\right\}^{-1 / 2}
\end{aligned}
$$

and similarly for $z_{t_{2}}$. 
$-73-$

Also

$$
\begin{aligned}
& S_{L}=\left(p_{a}-Q_{2}\right)^{2}=\left(k+k_{1}+\cdots+k_{r}\right)^{2} \\
& S_{R}=\left(p_{b}-Q_{1}\right)^{2}=\left(k+k_{r+1}+\cdots+k_{n}\right)^{2} \\
& M_{L}^{2}=\left(p_{a}+Q_{1}\right)^{2}=\left(k_{1}+\cdots+k_{r}\right)^{2} \\
& M_{R}^{2}=\left(p_{b}+Q_{2}\right)^{2}=\left(k_{r+1}+\cdots+k_{n}\right)^{2}
\end{aligned}
$$

We rewrite (6.12) in the form

$$
\begin{aligned}
& 2 k_{0} \frac{d \sigma}{d^{3} k}=\frac{(2 \pi)^{4}}{4 F_{a b}} \int d t_{1} \int d t_{2} \int d M_{L}^{2} \int d M_{k}^{2} \int d Q_{1} \int d Q_{2} \\
& \delta\left(t_{1}-Q_{1}^{2}\right) \delta\left(t_{2}-Q_{2}^{2}\right) \delta\left(\left(P_{a}+Q_{1}\right)^{2}-M_{L}^{2}\right) \\
& \delta\left(\left(P_{b}+Q_{2}\right)^{2}-M_{R}^{2}\right) \delta\left(Q_{1}+Q_{2}+k\right) \\
& \left|\gamma\left(Q_{1}, Q_{2}\right)\right|^{2}\left(z_{t_{1}}\right)^{2 \alpha_{1}\left(t_{1}\right)}\left(z_{t_{2}}\right)^{2 \alpha_{2}\left(t_{2}\right)} \\
& \sum_{n} \sum_{r}\left(\int \prod_{i=1}^{r} \frac{d^{3} k_{i}}{2 k_{0 i}} \delta\left(p_{a}+Q_{1}-k_{1}-\cdots-k_{r}\right)\left|\gamma_{a}\right|^{2}\right) \\
& \left(\int \prod_{j=r+1}^{n} \frac{d^{3} k_{j}}{2 k_{0 j}} \delta\left(p_{b}+Q_{2}-k_{r+1}-\cdots-k_{n}\right)\left|\gamma_{b}\right|^{2}\right)
\end{aligned}
$$

We may again convert the last integrals into cross sections for Reggeon + particle by inserting the appropriate flux factors. We get

$$
\text { 2 ko } \begin{aligned}
& \frac{d \sigma}{d^{3} k}=\frac{4}{(2 \pi)^{4} F_{a b}} \int d t_{1} d t_{2} d M_{L}^{2} d M_{R}^{2}\left|\gamma\left(Q_{1}, Q_{2}\right)\right|^{2} \\
&\left(z_{t_{1}}\right)^{2 \alpha_{1}\left(t_{1}\right)}\left(z_{t_{2}}\right)^{2 \alpha_{2}\left(t_{2}\right)} F_{a Q_{1}} F_{b Q_{2}} \\
& I\left(t_{1}, t_{2}, M_{L}^{2}, M_{R}^{2}\right) \sigma\left(M_{L}^{2}, t_{1}\right) \sigma\left(M_{R}^{2}, t_{2}\right)
\end{aligned}
$$


$-74-$

Here

$$
F_{a Q_{1}}=\left(\left(p_{a} \cdot Q_{1}\right)^{2}-m_{a}^{2} t_{1}\right)^{1 / 2}, F_{b Q_{2}}=\left(\left(p_{b} \cdot Q_{2}\right)^{2}-m_{b}^{2} t_{2}\right)^{1 / 2}
$$

and

$$
\begin{aligned}
I\left(t_{1}, t_{2}, M_{L}^{2}, M_{R}^{2}\right)= & \int d Q_{1} \delta\left(Q_{1}^{2}-t_{1}\right) \delta\left(\left(Q_{1}+k\right)^{2}-t_{2}\right) \\
& \delta\left(\left(p_{a}+Q_{1}\right)^{2}-M_{L}^{2}\right) \\
& \delta\left(\left(p_{b}-k-Q_{1}\right)^{2}-M_{R}^{2}\right)
\end{aligned}
$$

The integral may be evaluated. It is given by

$$
I\left(t_{1}, t_{2}, M_{L}^{2}, M_{R}{ }^{2}\right)=\frac{1}{16 \sqrt{1 \Delta 1}}
$$

where the determinant

$$
\Delta=\left|\begin{array}{cccc}
Q_{1}^{2} & Q_{1} \cdot k & Q_{1} \cdot p_{a} & Q_{1} \cdot p_{b} \\
k \cdot Q_{1} & \mu^{2} & k \cdot p_{a} & k \cdot p_{b} \\
p_{a} \cdot Q_{1} & p_{a} \cdot k & m_{a}^{2} & p_{a} \cdot p_{b} \\
p_{b} \cdot Q_{1} & p_{b} \cdot k & p_{b} \cdot p_{a} & m_{b}^{2}
\end{array}\right|
$$

is evaluated by computing its elements from the delta functions that enter into $I$. The physical region is limited to one sign of $\Delta(\Delta<0)$.

With sufficiently simple forms for the unknown cross sections and the Reggeon-Reggeon-particle 
Here

$$
F_{a Q_{1}}=\left(\left(p_{a} \cdot Q_{1}\right)^{2}-m_{a}^{2} t_{1}\right)^{1 / 2}, F_{b Q_{2}}=\left(\left(p_{b} \cdot Q_{2}\right)^{2}-m_{b}^{2} t_{2}\right)^{1 / 2}
$$

and

$$
\begin{aligned}
I\left(t_{1}, t_{2}, M_{L}^{2}, M_{R}^{2}\right)= & \int d Q_{1} \delta\left(Q_{1}^{2}-t_{1}\right) \delta\left(\left(Q_{1}+k\right)^{2}-t_{2}\right) \\
& \delta\left(\left(p_{0}+Q_{1}\right)^{2}-M_{L}^{2}\right) \\
& \delta\left(\left(p_{b}-k-Q_{1}\right)^{2}-M_{R}^{2}\right)
\end{aligned}
$$

The integral may be evaluated. It is given by

$$
I\left(t_{1}, t_{2}, M_{L}^{2}, M_{R}^{2}\right)=\frac{1}{\mid 6 \sqrt{1 \Delta 1}}
$$

where the determinant

$$
\Delta=\left|\begin{array}{cccc}
Q_{1}^{2} & Q_{1} \cdot k & Q_{1} \cdot p_{a} & Q_{1} \cdot p_{b} \\
k \cdot Q_{1} & \mu^{2} & k \cdot p_{a} & k \cdot p_{b} \\
p_{a} \cdot Q_{1} & p_{a} \cdot k & m_{a}^{2} & p_{a} \cdot p_{b} \\
p_{b} \cdot Q_{1} & p_{b} \cdot k & p_{b} \cdot p_{a} & m_{b}^{2}
\end{array}\right|
$$

is evaluated by computing its elements from the delta functions that enter into. I. The physical region is limited to one sign of $\Delta(\Delta<0)$.

With sufficiently simple forms for the unknown cross sections and the Reggeon-Reggeon-particle 
coupling, the integral may be evaluated (Silverman and Tan (70); Bali et al. (70)). The results are naturally somewhat model dependent, and look complicated. They do show limiting behavior and scaling, in the sense that $\lim _{s \rightarrow \infty} \rho_{1}(s, x, k)$ exists, if we take for the trajectories. $\alpha(0)=1$.

Recently a more model-independent way of deriving these (and other) results, has become available through the important observation of Mueller (70), that the inclusive cross sections are related to a part of the discontinuity of the matrix element for the reaction

$$
\mathrm{A}+\mathrm{B}+\mathrm{C}(\mathrm{k}) \longrightarrow \mathrm{A}+\mathrm{B}+\mathrm{C}(\mathrm{k})
$$

as shown in Fig. 23. The cross section may be written as

$$
\text { 2k. } \begin{aligned}
\frac{d \sigma}{d^{3} k} & =\frac{(2 \pi)^{4}}{4 F_{a b}} A\left(s, p_{a} \cdot k, p_{b} \cdot k\right) \\
& =\frac{(2 \pi)^{4}}{4 F_{a b}} \sum_{n}\left|M_{n}\left(s_{+}, M_{+}^{2}, \mu_{i+}^{2}, \ldots\right)\right|^{2}
\end{aligned}
$$

where we schematically wrote the r.h.s. as a sum over the squares of amplitudes for

$$
A+B \rightarrow C(k)+X_{n}
$$

The variables

$$
\begin{aligned}
\mathbf{s} & =\left(p_{a}+p_{b}\right)^{2} \\
M^{2} & =\left(p_{a}+p_{b}-k\right)^{2}
\end{aligned}
$$


$-76-$

and the set of variables that characterize a given state $x_{n}$, symbolized by

$$
\mu_{i}^{2}=\left(p_{a}+p_{b}-q_{1}\right)^{2}
$$

are to be evaluated just above the physical cut: the notation $s_{+}, \ldots$ is shorthand for $s+1 \epsilon, \ldots$. In addition to energy variables, which are timelike, and for which the prescription is necessary, there are momentum transfers which have values below their thresholds, and do not therefore need a prescription.

If the only singularities are the normal threshold singularities, then

$$
M_{n}^{*}\left(s_{+}, M_{+}^{2}, \ldots\right)=M_{n}\left(s_{-}, M_{-}^{2}, \ldots\right)
$$

This is known as hermitian analyticity. We may thus write

$$
\begin{aligned}
& A\left(s, p_{a} \cdot k, p_{b} \cdot k\right)=\sum_{n} M_{n}\left(s_{+}, M_{+}^{2}, \mu_{i+}^{2}, \ldots\right) M_{n}\left(s_{-}, M_{-}^{2}, \mu_{i-}^{2} \ldots\right) \\
& =\sum_{n} M_{n}\left(s_{+}, M_{+}^{2}, \mu_{i+}^{2} \ldots\right) M_{n}\left(s_{+}, M_{-}^{2}, \mu_{i+\cdots}^{2}\right) \\
& +\sum_{n} M_{n}\left(s_{+}, M_{+}^{2}, \mu_{i+}^{2} \ldots\right)\left\{M_{n}\left(s_{-}, M_{-}^{2}, \mu_{i-\ldots}^{2}\right)\right. \\
& \left.-M_{n}\left(s_{+}, M_{-}^{2}, \mu_{i+}^{2}, . .\right)\right\} \\
& =\operatorname{Disc}_{M^{2}} M(A+B+C(k) \rightarrow A+B+C(k)) \\
& +R\left(s, p_{0} \cdot k, p_{b} \cdot k\right)
\end{aligned}
$$


The first term is identified as the discontinuity in $\mathrm{M}^{2}$ of the ampiitude for the forward reaction

$$
A+B+C(k) \longrightarrow A+B+C(k)
$$

This identification is particularly easy to make if we go into a region of the variables in which only $M^{2}$ lies above a physical threshold. In that case $\dot{s}_{+}=s_{-}$etc. since all of these variables lie in a region in which there is no cut. According to. Stapp (71) and Tan (71) who have analyzed this problem with some care, it is possible to start in this region (in which the remainder term $R\left(s, p_{a} \cdot k, p_{b} \cdot k\right)$ vanishes) and find a path of analytic continuation to physical values of $\mathbf{s}$, etc. such that the remainder term never appears. This is a rather delicate problem that we are not prepared to discuss here; Tan suggests that it is possible that only for the graph structure implied by the multiperipheral model (and dual resonance models) that such a continuation is straightforward. It is possible to extend the argument to inclusive reactions in which more than one particle is detected, i.e. $C(k)$ is replaced by several particles. The connection with the S-matrix suggests that an expansion of $A\left(s, p_{a} \cdot k, p_{b} \cdot k\right)$ in $O(2,1)$ harmonics be possible. For large $s, p_{a} \cdot k$ of $O(s)$ and $p_{b} \cdot k$ finite, one is dealing with target fragmentation. The procedure is:

This is the technical name for what we have been calling the crossed channel partial wave expansion. The basic theory is due to Toller (65). For further discussion see Jones et al (71) and Abarbanel (71). 
$-78-$

(i) Choose the frame in which particle $B$ is at rest, i.e. the laboratory frame. In that frame

$$
\begin{aligned}
& p_{a}=\left(m_{a} e^{Y}, 0,0, m_{a} e^{-Y}\right) \\
& p_{b}=\left(m_{b}, 0,0, m_{b}\right) \\
& k=\left(m_{\perp} e^{y}, k_{\perp}, 0, m_{\perp} e^{-y}\right)
\end{aligned}
$$

and

$$
\begin{aligned}
s & =m_{a} \cdot m_{b} e^{Y} \\
M^{2} & \cong m_{a} e^{Y}\left(m_{b}-m_{\perp} e^{-y}\right) \simeq s\left(1-\frac{m_{\perp}}{m_{b}} e^{-y}\right) \\
p_{b} \cdot k & =m_{b} m_{\perp} \cosh y \\
p_{a} \cdot k & =m_{a} m_{\perp} \cosh (Y-y)
\end{aligned}
$$

(ii) Following Jones et al. (71) write the $O(2,1)$ expansion

$$
\begin{array}{r}
A=\int d \mu \int d \lambda \sum_{\rho=1}^{2} A_{i \mu, \rho}^{\lambda}\left(y, k_{\perp}\right) \\
d_{i \mu, \rho}^{\lambda}(Y)
\end{array}
$$

The labels if and $\lambda$ may be interpreted as analytic continuations of telicity differences and angular momentum, respectively in the $A \bar{A}$ channel. Since we will not discuss predictions of spin dependence in this brief summary (see Abarbanel and Gross (71)) our only concern is with the asymptotic behavior 


$$
\operatorname{di\mu ,\rho }_{i \lambda}^{\lambda}(Y) \sim e^{\lambda Y}
$$

With an appropriate assumption about the singularities of $A_{i p . s}^{\lambda}(y, k)$ in the $\lambda$ plane, a deformation of contours similar to the one familiar from two-body Regge theory suggests the asymptotic form

$$
A\left(s, p_{a} \cdot k, p_{b} \cdot k\right) \sim s^{\alpha} f\left(y, k_{\perp}\right)
$$

If $\alpha=1$, corresponding to Pomeron-like behavior, then (6.22) will have a limiting distribution, since the factors of $s$ cancel. ${ }^{+}$If the next singularity in the $\lambda$ plane yields a secondary $\alpha \approx 1 / 2$ as in two-body reactions, then

$2 k_{0} \frac{d \sigma}{d^{3} k}=\rho_{1}\left(k_{2}, \underline{k}\right)+\frac{m_{0}}{s^{1 / 2}} \rho_{1}^{\prime}\left(k_{2} \cdot \underset{\sim}{k}\right)$

i.e. limiting behavior is approached quite rapidly. In the pionization region, both $p_{a} \cdot k$ and $p_{b} \cdot k$ are large, of order $s^{1 / 2}$. Here Mueller suggests a double I If, instead of a Pomeron-like pole, there is a cut, then an asymptotic behavior like $s(\log s)^{\beta}$ could occur. This implies a violation of limiting behavior, but not necessarily an unphysical situation (cf. Fig. 8a). In the presence of cuts the factorization of the function $f\left(y, k_{\perp}\right)$ into a term that depends on particle $A$ and one that depends on BC would also.be violated. This will be testable. 
$-80-$

expansion. Here we work in the rest frame of particle $C$ and take

$$
\begin{aligned}
k= & (\mu, 0,0, \mu) \\
p_{b}= & \left(m_{b} e^{-y_{2}}, 0,0, m_{b} e^{y_{2}}\right) \\
p_{a}= & m_{a}\left(\operatorname{ch} y_{1}+\operatorname{sh} y_{1} \cos \phi, \operatorname{sh} y_{1} \sin \phi, 0,\right. \\
& \left.\quad c h y_{1}-\operatorname{sh} y_{1} \cos \phi\right)
\end{aligned}
$$

the latter to conform with Mueller's notation. An expansion of the type

$$
\begin{gathered}
A=\iiint \int d \mu d \mu^{\prime} d \lambda d \lambda^{\prime} \sum_{\rho \rho^{\prime}} A_{i \mu, \ldots}^{\lambda \lambda^{\prime}}(\phi) \\
d_{i \mu, \rho}^{\lambda}\left(y_{1}\right) d_{i \mu^{\prime}, \rho^{\prime}}^{\lambda^{\prime}}\left(y_{2}\right)
\end{gathered}
$$

$(6.30)$

yields the asymptotic behavior

$$
A\left(s, p_{a} \cdot k, p_{b} \cdot k\right) \approx e^{y_{1} \alpha_{1}+y_{2} \alpha_{2}} f(\phi)
$$

We note that

$$
\begin{aligned}
& s \approx 2 m_{1} m_{b}\left(\operatorname{ch} y_{1} c h y_{2}+s h y_{1} \operatorname{sh} y_{2} \cos \phi\right) \\
& p_{0} \cdot k=m_{0} \mu c h y_{1} \\
& p_{b} \cdot k=m_{b} \mu c h y_{2}
\end{aligned}
$$

Furthermore, a little algebra shows that

$$
\cos \phi=\frac{\mu^{2}-k^{2}}{\mu^{2}+k^{2}}
$$


Hence if $\alpha_{1}=\alpha_{2}=1$, we have $A \sim s f(\phi)$ i.e.

$$
2 k_{0} \frac{d \sigma}{d^{3} k} \rightarrow f\left(\underset{\sim}{k^{2}}\right)
$$

It is not clear, of course, whether double Pomeron exchange is permitted here, when it seems to be forbidden in the multiperipheral graph structures, but that is not a question we are prepared to discuss here.

The method outlined above can be used to discuss the behavior near the minematic boundary (DeTar et al.(71)) and to make some predictions about the spin dependence (Abarbanel and Gross (71)). We shall content ourselves with this impressionistic glimpse of what promises to be the new phenomenology of many-particle reactions, except to draw attention to some observations of Chan et al. (71):

The two terms in $(6.28)$ have their origin in different Regge poles, with $\rho_{1}$ coming from Pomeron exchange and $\rho_{1}^{\prime}$ from $P^{\prime}$ and $\rho$ exchange. In the reactions

$$
\pi^{ \pm}+p \rightarrow C+\text { anything }
$$

where $C$ is a fragment of the target,

$$
\text { 2k. } \frac{d \sigma^{ \pm}}{d^{3} k}=\rho_{1}+\frac{\mu}{s^{1 / 2}}\left(\rho_{1}^{\prime}\left(p^{\prime}\right) \pm \rho_{1}^{\prime}(\rho)\right)
$$

the change in sign coming from the coupling of $\rho$ to $\pi^{ \pm}$. Thus the relation

$$
2 k^{*}\left(\frac{d \sigma^{+}}{d^{3} k}-\frac{d \sigma^{-}}{d^{3} k}\right)=\frac{2 \mu}{s^{1 / 2}} \rho_{1}^{\prime}(\rho)
$$


Similar conclusions can be drawn for pairs of reactions initiated by $\mathrm{K}^{+}$and $\mathrm{K}^{-}$, or $\mathrm{p}$ and $\overline{\mathrm{p}}$ as projectiles. One may also extend duality, in the form of the association of exotic quantum numbers in a physical channel with energy independence of the discontinuity, to inclusive reactions. There does not, as yet, exist a counterpart of Finite Energy Sum Rules for three-particle $S$ matrix elements, but it is possible to draw duality diagrams. When this is done with some care in identifying which are the timelike channels ${ }^{+}$, then the rule emerges that the cross sections in which the quantum numbers of $(A+B)$ and $(A+B+\bar{C}), i . e$. the "summed over" channel, are exotic, are constant in the same way that the $\mathrm{K}^{+} \mathrm{p}$ cross section is constant. For example

$$
\mathrm{K}^{+}+\mathrm{p} \rightarrow \pi^{ \pm}+\text {anything }
$$

should have a cross section that is constant from energies of $2 \mathrm{GeV} / \mathrm{c}$ up, if $\mathrm{K}^{+} \mathrm{p}$ is any guide.

In our discussion of inclusive reactions, we have concentrated on

$$
\mathrm{A}+\dot{\mathrm{B}} \longrightarrow \mathrm{C}+\text { anything }
$$

because these are easiest to measure. With the development of the Mueller-Regge phenomenology, some fairly well

\footnotetext{
Ellis et al. (71) point out that the rule of chan et al. (71) which did not require that $A B$ also be exotic leads to a contradiction, if factorization is used.
} 
defined predictions concerning more complicated inclusive reactions such as

$$
A+B \rightarrow C\left(k_{1}\right)+C\left(k_{2}\right)+\text { anything }
$$

emerge $^{+}$. Our sketchy treatment of the formalism of the $O(2,1)$ expansions does not allow us a detailed discussion of such predictions.

\section{ACKNOWLEDGEMENTS}

I would like to thank my colleagues at the University of Minnesota for discussions and questions raised during the lecture series that gave rise to this report. I learned much from discussions with F.Yen at Argonne National Laboratory and L.S.Brown at the Aspen Center for Physics. I am also grateful to T.Ferbel, C.Quigg and L.L.Wang for sending me copies of their reports before publication.

\footnotetext{
The first paper on this subject has just appeared (Freedman et al. (71)). See also Abarbanel (71) and, for a more qualitative discussion, Wilson (70).
} 
FIGURE CAPTIONS

Fig.l The multiperipheral graph that defines the variables used in the discussion of the model.

Fig.2 (a) Graphical representation of the reaction $\mathrm{p}+\boldsymbol{\pi}^{-} \rightarrow \pi^{+}+\pi^{-}+\mathrm{n}$ for low momentum transfer between the proton and the neutron. (b) Multiperipheral representation of the reaction $\mathrm{p}+\pi^{-} \rightarrow \pi^{+}+\pi^{-}+\mathrm{n}$, with the off-shell pion-pion scattering described by $\rho$ exchange.

Fig.3. Multiperipheral graph for many-pion production dominated by pion exchange.

Fig.4 Typical distribution of transverse momenta in $\pi^{-} p$ at $25 \mathrm{GeV} / \mathrm{c}$, from Elbert et al. (68).

Fig.5. A comparison of the experimental $\pi^{+} \pi^{-}$ elastic cross-section with an extranolated Regge fit, from Fox (59).

Fig.6 A multiperipheral cluster Eraph.

Fig.7 (a) Fragmentation in the center of mass frame. (b) Fragmentation with pionization in the center of mass frame. C describes the pionization products.

Fig.8 (a) One particle distribution for two values of $\mathrm{Y}$ (with $\mathrm{Y}_{2}>\mathrm{Y}_{1}$ ) for a "fireball" model.

(b) One particle distribution for a hadronic breakup model shown in Fig. 7 b.

(c) One particle distribution with a multiperipherai component. 
Fig.9 The pion distributions measured by Anthony et al. (71), plotted as a function of incident energy and $x=2 k_{z}^{*} / s^{1 / 2}$. The dashed lines are handdrawn through the points of equal $x$. (From Chen et al. (71)).

Fig.10 Distribution of $\sigma / \mathrm{dk}_{z}$ for $\pi^{-}$in $\mathrm{p}-\mathrm{p}$ collisions from the analysis of Chou and Yang(70). The dotted, dot-dashed and solid curves are for 12.9, 21.1 and $28.4 \mathrm{GeV} / \mathrm{c}$ incoming momenta. Fig.11 Plot of $\sigma / \mathrm{dk}_{\mathbf{z}}^{*} \mathrm{~d}^{2} \mathrm{k}$ from Anderson et al. (67) as a function of $\mathrm{k}_{\mathrm{z}}^{*}$. The pion cross section curve is the one with the strong $k_{z}^{*}$ dependence. Fig.12 Plot of $d \sigma / d x$ for $\pi^{-}$produced in $\mathrm{K}^{+} \mathrm{p}$ collisions at $11.8 \mathrm{GeV} / \mathrm{c}$. Solid line is fit to data. Dashed line represents $25 \mathrm{GeV} / \mathrm{c} \pi^{-} \mathrm{p}$ data of Elbert et al.(71). The numbers represent slope parameters.

Fig.13. Longitudinal momentum distribution of $\pi^{-}$in the "quark model" center of mass frame (from Stone et al.(71)).

Fig.14 Cross sections for two-body diffractive reactions from Anderson et al.(66). Note fall off of nondiffractive $p+p \rightarrow p+N^{*}(1240)$.

Fig.15 Spectra of $M\left(\pi^{+} \pi^{-} \pi^{-}\right)$from the reaction

$$
\pi^{-}+p \rightarrow(3 \pi)^{-}+p \text { at } 13 \text { and } 20 \mathrm{GeV} / \mathrm{c} \text {, }
$$

from Ioffredo et al. (68) 
Fig.16 Four possible correlations of the $\pi^{+} \pi^{-} \pi^{-}$in the final state of $\pi^{-} p \rightarrow 2 \pi^{-} \pi^{+} p$. The arrows are drawn to show the forward (to the right) and backward (to the left) directions in the c.m.s. Cases (a), (b) require a charge exchange; cases (c), (d) can proceed diffractively.

Fig.17 Momenta in diffraction excitation of projectile. The same graph describes target fragmentation near its kinematic limit $k^{-}=m_{b}$. The exchange may, in that case, not be the Pomeron, since particle $c$ may be different from $b$ in its internal quantum numbers.

Fig.18 Graphical relation between inclusive cross section and three-Reggeon coupling. "Disc ${ }_{M} 2$ " means that the absorptive part of the three-Reggeon matrix element is to be taken, with the discontinuity in the variable $M^{2}=\left(p_{a}+p_{b}-k\right)^{2}$

Fig.19 Plot of d $\sigma / d$ tdM against $M$ for $n^{-}+p \rightarrow$ $\pi^{-}+$anything, at 8 and $16 \mathrm{GeV} / \mathrm{c}$. The data is from Anderson et al. (70)

Fig.20 Plot of d $\sigma / d$ tdM against $M$ for $p+p \rightarrow$ $p+$ anything, at $6.1,9.9,15.1$ and $20.0 \mathrm{GeV} / \mathrm{c}$. The data is from Anderson et al. (66)

Fig.21 Plot of $d \sigma / d M d \Omega_{\text {lab }}$ against $M$ for backward $p$ in $\pi^{-}+p \rightarrow p+$ anything, at 8 and $16 \mathrm{GeV} / \mathrm{c}$. The data is from Anderson et al. (69) 
Fig. 22 Graphical representation of matrix element for the emission of a particle from a multiperipheral graph.

Fig. 23 Graphical representation of inclusive cross section for $A+B \rightarrow C+$ anything as a discontinuity in the variable $\mathrm{M}^{2}=\left(\mathrm{p}_{\mathrm{a}}+\mathrm{p}_{\mathrm{b}}-\mathrm{k}\right)^{2}$ in the amplitude for $A+B+C \rightarrow A+B+C$. 


\section{References}

AAchen-Berlin-Bonn-CERN-Cracow Collaboration:

Boesebeck, K., Grassler, H., Kraus, G., Schulte, R., Bottcher, H., Kaltwasser, J., Kautmann, H., Nowak, S., Bockmann, K., Bossen, J. G., Drevermann, H., Johnssen W., Rost, M., Sternberger, K., stocker, U., Angelopoulos, A., Campbell, J.R., Cocconi, V. T., Hansen, J. D., Kittel, W., Matsumoto, S., Morrison, D. R. O., stroynowski, R., Whittaker, J. B. and Loskiewicz, J., Nuclear Physics (to be published)

Abarbanel, H. D. I., Phys. Rev. D3, 2227 (1971).

Abarbanel, H. D. I., Phys. Letters, 34B, 67 (1971). Abarbanel, H. D. I., Chew, G. F., Goldberger, M. L. and Saunders, L. M., Phys. Rev. Letters, 26, 937 (1971). Abarbanel, H. D. I. and Gross, D. J., Phys. Rev. Letters, 26, $732(1971)$

Abolins, M. A., Smith, G. A., Ma, Z. M., Gellert, E. and wicklund, A. B., Phys . Rev. Letters, 25, 126 (1970). Amati, D., Stanghellini, A. and Fubini, S. Nuovo Cimento, 26, 6, (1962)

Anderson, E. W., Bleser, E. J., Blieden, H. R., Collins, G. B., Garelick, D., Menes, J.; Turkot, F., Birnbaum, D., Edelstein, R. M., Hien, N. C., McMahon, T. J., Mucci, J. and Russ, J., Phys. Rev. Letters, 22, 1690 (1969). 
Anderson, E. W., Bleser, E. J., Blieden, H. R., Collins, G. B., Garelick, D., Menes, J., Turkot, F., Birnbaum, D., Edelstein, R. M., Hien, N. C., McMahon, T. J., Mucci, J. F. and Russ, J. S., Phys. Rev. Letters, 25, 699 (1970). Anderson, E. W., Bleser, E. J., Collins, G. B., Fujii, T., Menes, J., Turkot, F., Carrigan, Jr.R. A., Edelstein, R. M., Hien, N. C., McMahon; T. J. and Nadelhaft, I., Phys. Rev. Letters, 16, 855 (1966). Anderson, E. W., Bleser, E. J., Collins, G. B., Fujii, T., Menes, J., Turkot, F., Carrigan, Jr.R. A., Edelstein, R.M., Hien, N. C., McMahon, T. J., and Nadelhatt, I., Phys. Rev. Letters, 19 , 198 (1967). Anthony, R. W., cotiin, C. T., Meanley, E. S., Rice, J. E., Terwilliger, K. M. and stanton, N. R., Phys: Rev. Letters, 26, $38(1971)$.

Ascoli, G., Brockway, D. V., Eisenstein, L., Iotiredo, M. L., Kruse, U. E., Schultz, P. F., Caso, C., Tomasini, G., von Handel, P. Schilling, P., costa, G., Ratti, S., Daronian, P., Mosca, L., Breener, A. E., Harrison, W. C. Heyda, D., Johnson Jr., W. H., Kim, J. K., Law, M. E., Mueller, J. E., Salzberg, B. M., Sisterson, L. K., Johnston, T. F., Prentice, J. D.,. Steenberg, N. R., Yoon, T. S., Carroll, J. T., Erwin, A. R., Morse, R., Oh, B. Y., Roberston, W. and Walker, W. D., Phys. Rev. Letters, 26929 (1971). 
Bali, N. F., Brown, I. S., Peccei, R. D., and Pignotti, A., Phys. Rev. Letters 25, 557 (1970).

Bali, N. F., Chew, G. F. and Pignotti, A., Phys. Rev. Letters, 19, 614 (1967); Phys. Rev. 163, 1572 (1967). Bali, N. F., Pignotti, A. and steele, D., Phys. Rev. Benecke, J.; Chou, T. T., Yang, C. N., and Yen, E., Phys. Rev. 188, 2159 (1969). Bertocchi, L., Fubini, S. and Tonin, M., Nuovo Cimento, $\underline{25}, 626(1962)$

Berger, E. L., Phys. Rev. 179, 1567 (1969).

Biswas, N. N., Cason, N. M., Kenney, V. P., Powers, J. T., Shephard, W. D. and Thomas, W. D., "Longitudinal and Transverse Momentum Distributions for $\pi^{-}$Mesons in $18.5 \mathrm{GeV} / \mathrm{c} \pi^{ \pm} \mathrm{p}$ Interactions" (to be published). Byers, N. and Frautschi, s. "Diffraction Dissociation in the Cou-Yang Model", published in "Quanta", C. Goebel (Ed) University of Chicago Press, 1970.

Caneschi, L. and Pignotti, A., Phys. Rev. Lett. 22, 1219 (1969). Caneschi, L. and Schwimmer, A., Phys. Letters, 33B, 557 (1970). Chan Hong-Mo, Hsue, C. S., Quigg, C. and Wang, J. M., Phys. Rev. Letters, 26, 672 (1971).

Chang, I. N., Freund, P. G. O. and Nambu, Y., Phys. Rev. Letters, $\underline{24}, 628$ (1970).

Chang, S. J. and Ma, S. K., Phys. Rev. 180, 1506 (1969); 188, 2385 (1969). 
Chang; S. J. and Yan; T. M., Phys. Rev. Letters, 25; 1586 (1970)

Chen, M. S., Wang, L. L. and Wong, T. F., Phys. Rev. Letters, 26, 280 (1971)

Cheng, H. and Wu, T. T., Phys. Rev. Letters, 24, 1456 (1970). Chew; G. F. and Pignotti, A., Phys. Rev. 176, 2112 (1968). Chew, G. F., Rogers, T. and snider, D. R., Phys. Rev. D2, 765 (1970)

Chou, T. T., and Yang, C. N., Phys. Rev. Letters, 25, 1072 (1970)

DeTar, C., Phys. Rev. D3, 128 (1971).

DeTar, C. S., Jones, C. E., Low, F. E., Weis, J. H., Young, J. E. and Tan, C. I., Phys. Rev. Letters, 26, 675 (1971)

Diddens, A. N., Galbraith, W., Lillethun, E., Manning, G., Parham, A. G., Taylor, A. E., walker, T. G. and Withẽrell, A. M. Nuovo Cimento, $\underline{31}, 961$ (1964). Elbert, J. W., Erwin, A. R., Mikamo, S., Reeder, D., Chen, Y. Y., Walker, W. D., and Weinberg, A., Phys. Rev. Lietters, 20, $124 \cdot(1968)$. Elbert, J. W., Erwin, A. R. and Walker, W. D., "Evidence for the Internal structure of Hadrons obtained from Multipion Production", Phys. Rev. D3; 2042 (1971) . 
Elbert, J. W., Erwin, A. R., Walker, W. D. and wäters, J.W. Nuclear Physics, B19; 85 (1970).

Feynman, R. P., "High Energy Collisions", C. N. Yang, et.al. Ed. Gordon and Breach, Science Publishers, New York, 1969. Finkelstein, J. and Kajantie, K., Nuovo Cimento, 56A, 659 (1968); Phys. Letters, 26B, 305 (1968).

Fox, G., "High Energy Collisions", C. N. Yang, et.al., Ed. Gordon and Breach, Science Publishers, New York, 1969. Franzini, P. in "High Energy Collisions", C. N. Yang, et.al. Ed., Gordon and Breach, Publishers, 1969.

Freedman, D. Z., Jones, C. E., Low, F. E. and Young, J. E., Phys. Rev. Letters, 26, 1197 (1971).

Freund, P. G. O. Phys. Rev. Letters, 20, 235 (1968).

Fubini, S. in "Strong Interactions and High Energy Physics", Edinburgh (1963).

Goebel, C. J. Proc. Int. Conf. on Theoretical Aspects of Very High Energy Phenomena, CERN, 1961 (CERN report 61-22). Good, M. L. and Walker, W. D., Phys. Rev. 120; 1857 (1960)

Gribov, V. N. Yadernaya Fiz.'(U.S.S.R.) 5, 197 (1967) . Halliday, I. G. and Saunders, I. M. Nuovo Cimento, 60A, 115 (1969).

Harari, H., Phys. Rev. Letters, 20, 1395 (1968). 
Horn, D. and Silver, R., "Coherent Production of Pions" to be published in Annals of Physics (N. Y.). Hwa, R. C. Phys - Rev. Letters, 26, 1143 (1971). Huson, R., Lubatti, H. J., Six, J., Veillet, J. J., Annoni, H., Bellini, G. Di Corato, M., Fiorini, E., Moriyasu, K.; Negri, P., Roller, M., Bingham, H. H., Farwell, C. W. and Fretter, W. B., Phys. Letters, 28B, 208 (1968).

Iotiredo, M. L., Brandenburg, G. W., Brenner, A. E., Eisenstein, B., Eisenstein, L., Johnson, Jr., W. H., Kim, J. K., Law, M. E., Salzberg,, B. M., Scharenguivel, J. H., Sisterson, L. K. and Szymanski, J. J., Phys. Rev. Letters, 21, 1212 (1968).

Jones, C. E., Low, F. E. and Young, J. E., "Generalized $O(2,1)$. Expansion for Asymptotically Growing Amplitudes" to be published in Ann. Phys. (N. Y.) vol. 66.

Jones, L. W., Bussian; A. E., DeMeester, G. D., Loo, B. W., Lyon Jr., D. E., Ramana Murthy, P. V., Roth, R. F., Learned, J. G., Mills, F. E., Reeder, D. D., Erickson, K. N. and cork, B., Phys. Rev. Letters, 25, 1679 (1970). Ko, W. and Lander, R. L., Phys. Rev. Letters, 26, 1064 (1971).

Kogut, J. B. and Soper, D. E., Phys. Rev. Dl, 2901 (1970) . 
Lipes, R., Zweig, G. and Robertson, W., Phys. Rev. Letters, 22, 433 (1969).

Morrison, D. R. O. CERN/TC/PHYSICS 66-20, 1966 (unpublished). Morrison, D. R. O., Phys. Rev. 165, 1699 (1968). Mueller, A. H., Phys. Rev. D2, 2963 (1970).

Peccei, R."D. and Pignotti, A., Phys. Rev. Letters, 26, 1076, (1971).

Quigg, C., "Multiparticle Reactions at Itermediate Energies" Invited paper at the Pasadena Meeting on Hadron Physics at Intermediate Energies, 1971.

Rajàraman, R., Phys. Rev. Dl, 118 (1970).

Ranit, G., Fort.d.Physik, 18, I (1970) .

Ranft, J., Phys. Letters, 31B, 529 (1970).

Silverman, D. and Tan, C. I., Nuovo Cimento,

Silverman, D. and Tan, C. I., Phys. Rev. D2, 233 (1970).

Smith, D. B., Sprarka, R. J., and Anderson, J. A., Phys.

Rev. Letters, 23, 1064 (1969).

Stapp,H.P., "Inclusive cross sections as Discontinuities", to be published (1971).

stone, S. I., Cohen, D., Farber, M., Ferbel, T., Holmes, R., slattery, 'P. and Werner, B., "Longitudinal Momentum Distributions of $\pi^{-}$Mesons produced in $12.7 \mathrm{GeV} / \mathrm{c} \mathrm{K}^{+} \mathrm{p}$ and $7 \mathrm{GeV} / \mathrm{c} \pi^{+} \mathrm{p}$ interactions". (to be published). 
sudakov, V. V., zurn. Eksp. Teor. Fiz. 30, 87. (1956).

Tan, C. I., "Discontinuity Formulae and a Derivation of Mueller's Regge Hypothesis for Inclusive Cross sections.", to be published (1971).

Ting, P. D. and Yesian, H. J., "Triple Pomeron Vertex, Duality and Difiraction Dissociation", to be published (1971)

Toller, M., Nuovo Cimento 37,631 (1965).

Turkot, F. in Proc. Topical Conference on High-Energy Collisions of Hadrons, CERN, 1968 (CERN report 68-7/1). Van Hove; L. in "Proceedings of the colloquium on High Multiplicity Hadronic Interactions", A. Krzywicki, et.al., Ed. Ecole Polytechnique, Paris (1970).

Van Hove, L., Nuclear Physics, B9, 331 (1969).

Wang, J. M. and Wang, L. L., "A Phenomenology of the Background in the Resonance Production Region", to be published (1971)

Wilson, K., Acta Phys. Austr. 17, 37 (1963).

Wilson, K. G., "Some Experiments on Multiple Production", to be published (1970).

Zachariasen, F. and Zweig, G., Phys. Rev. 160, 1322 (1967). Zachariasen, F. and Zweig, G., Phys. Rev. 160, 1326 (1967). 
Good, M. L. in Proceedings of the Conterence on Expectations for Particle Reactions at the New Accelerators, Barger, V. and Durand, L, Ed., Madison, Wisconsin (1970). Ellis, J., Finkelstein, J., Frampton, P. H. and Jacob, M., "Duality and Inclusive Phenomenology" CERN TH-1316 to be published (1971). 


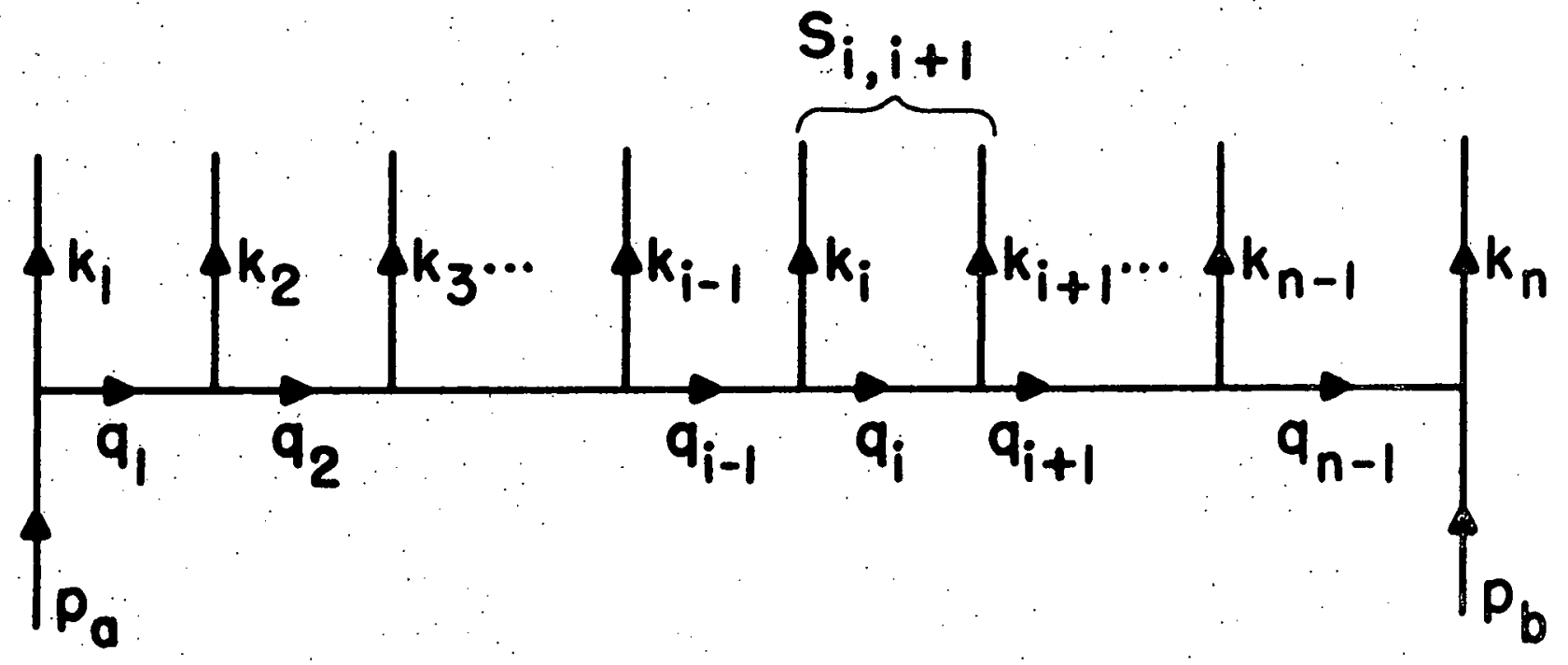

Fig. I 


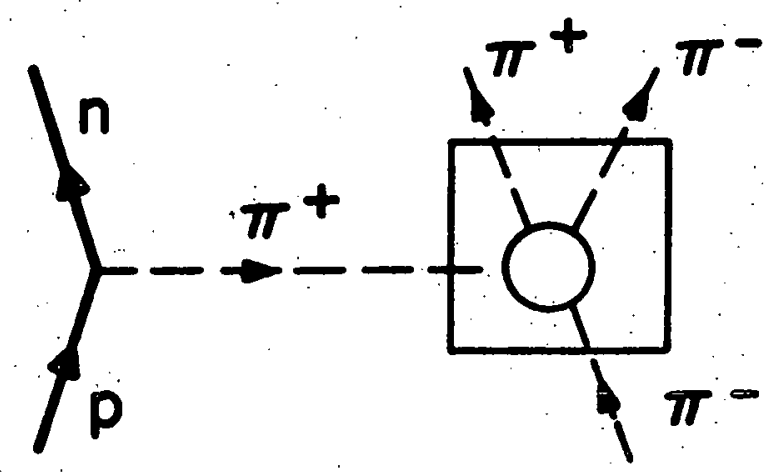

Fig. $2 a$

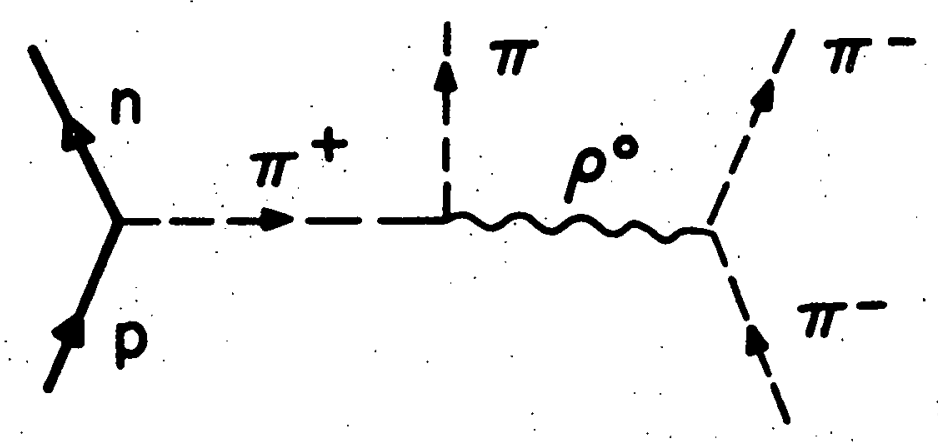

Fig. $2 b$

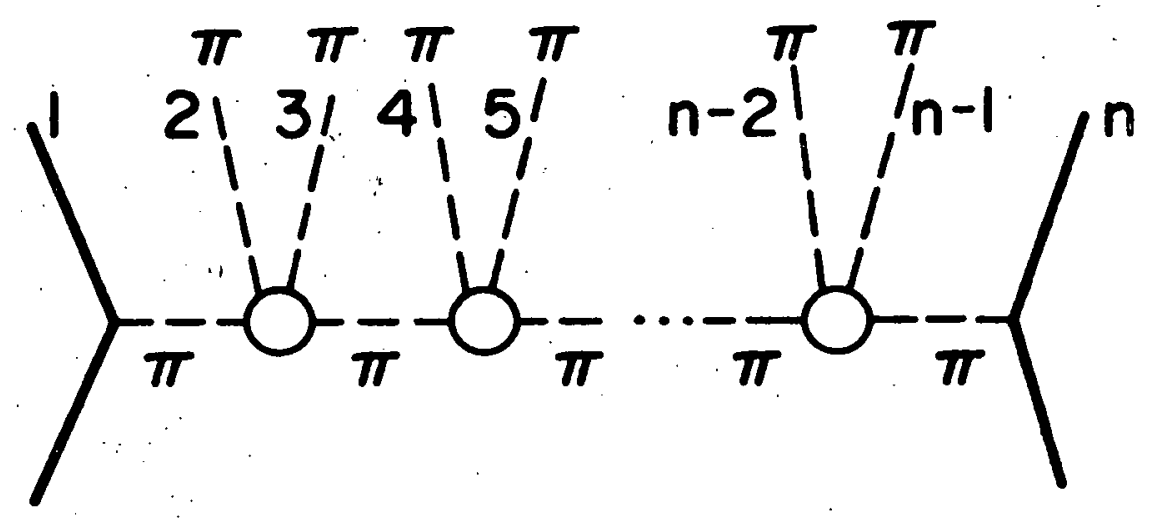

Fig. 3 


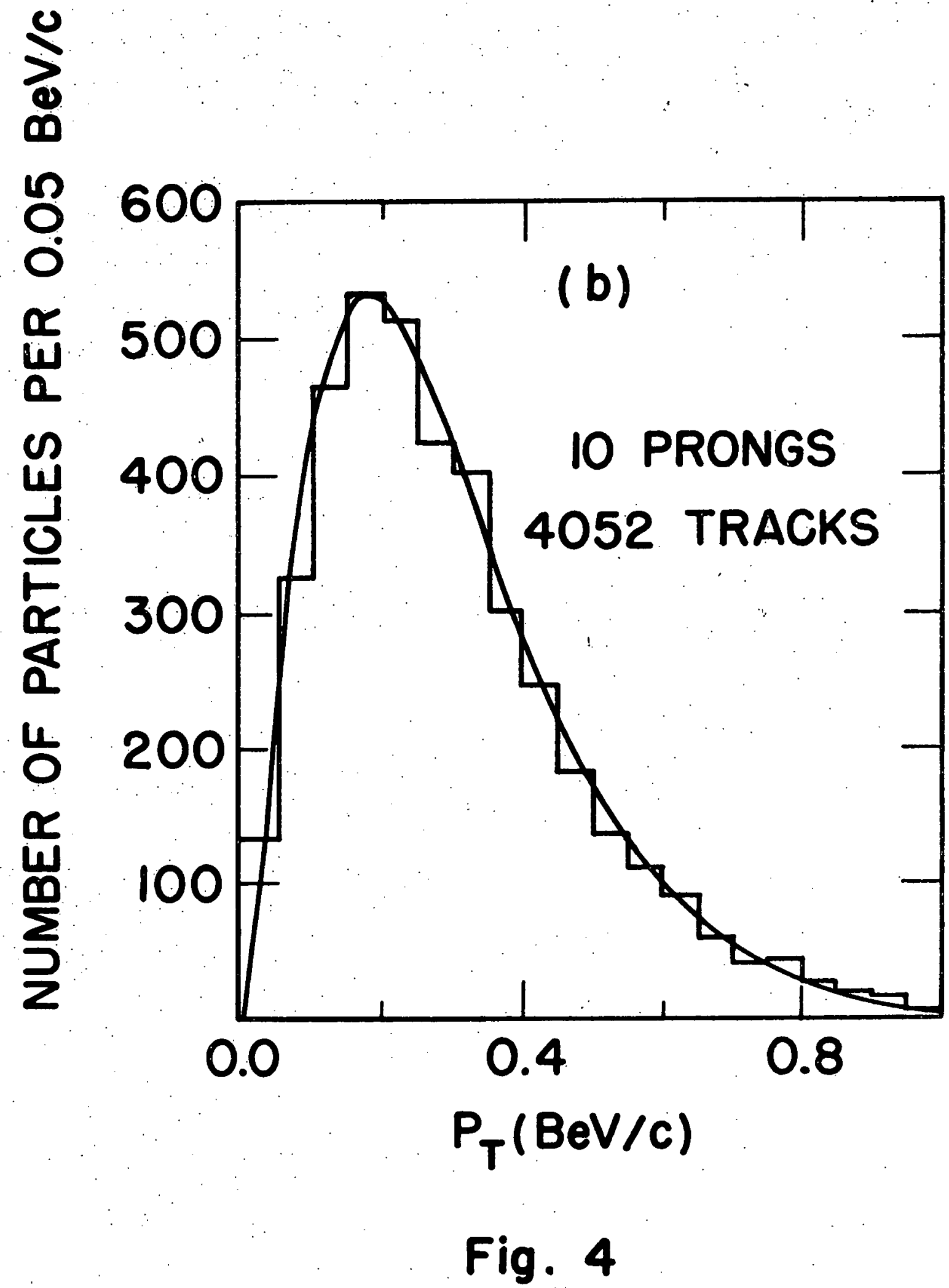




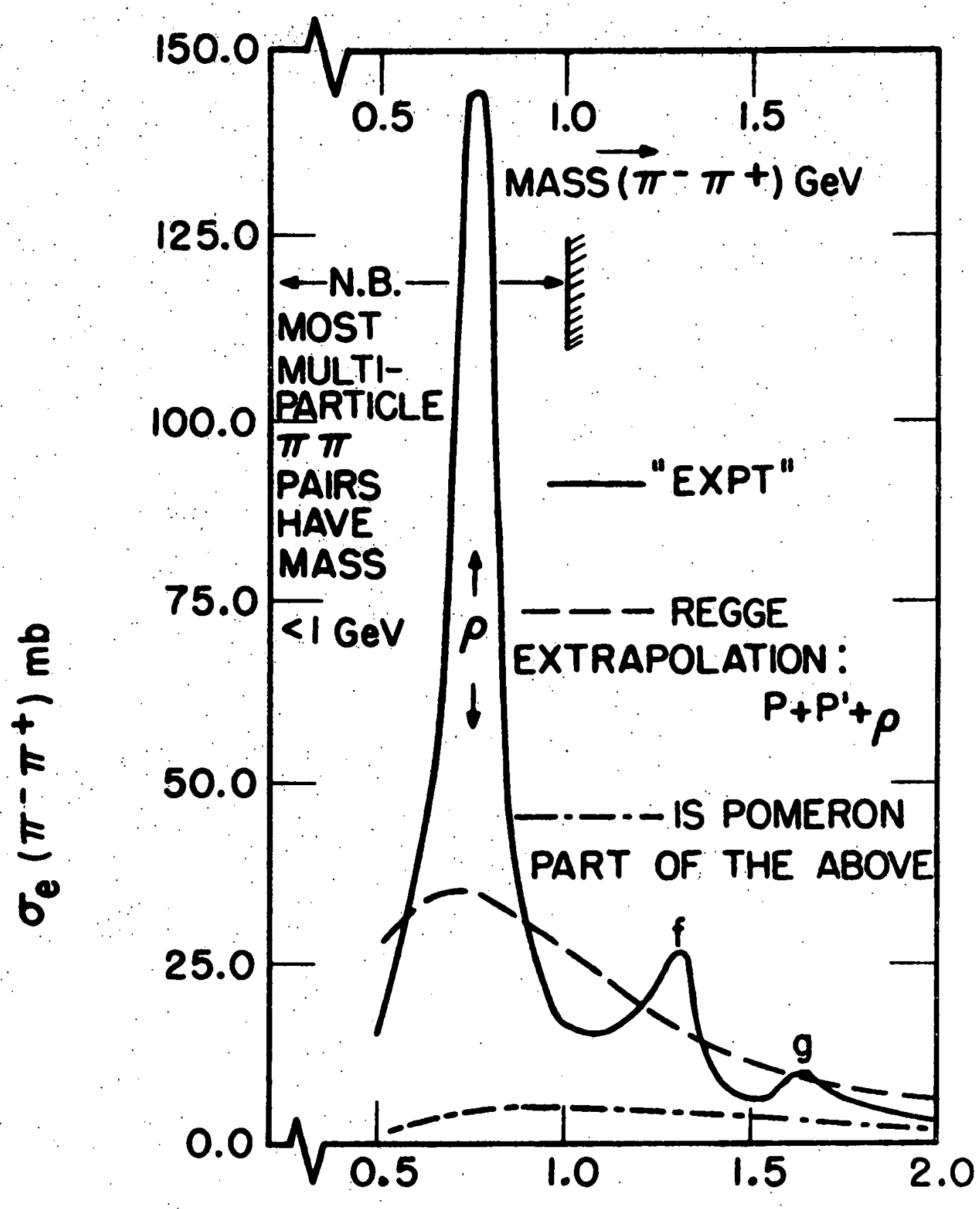

Fig. 5 


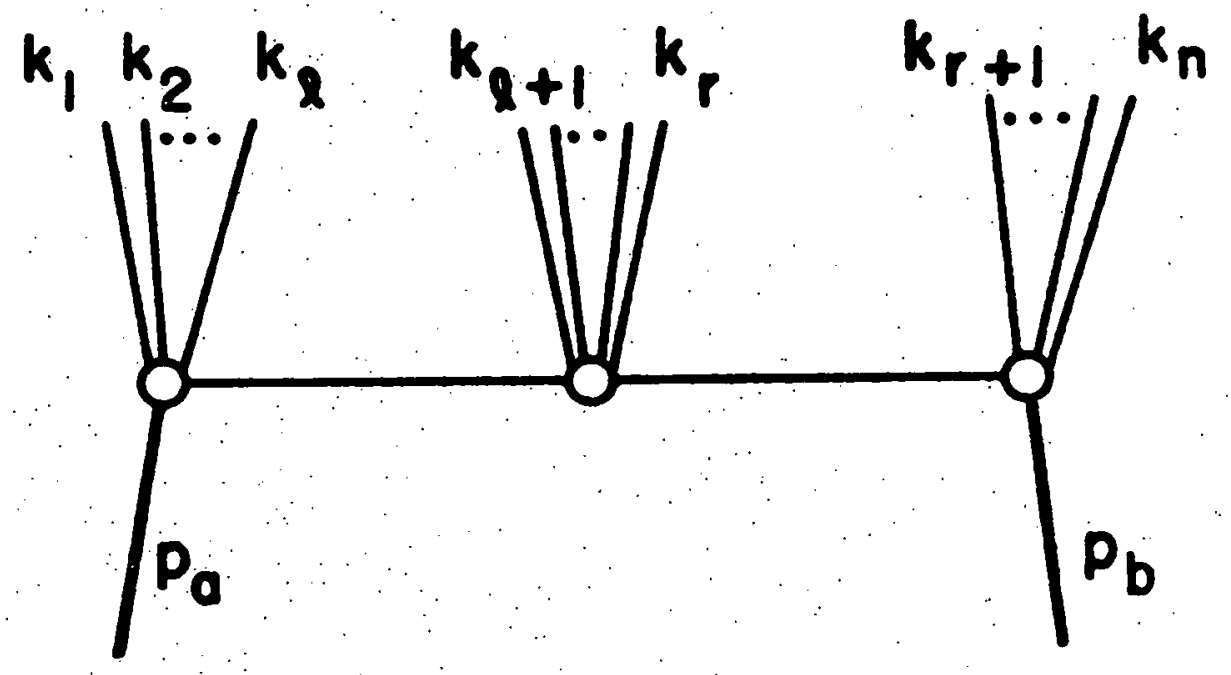

Fig. 6 
(a)
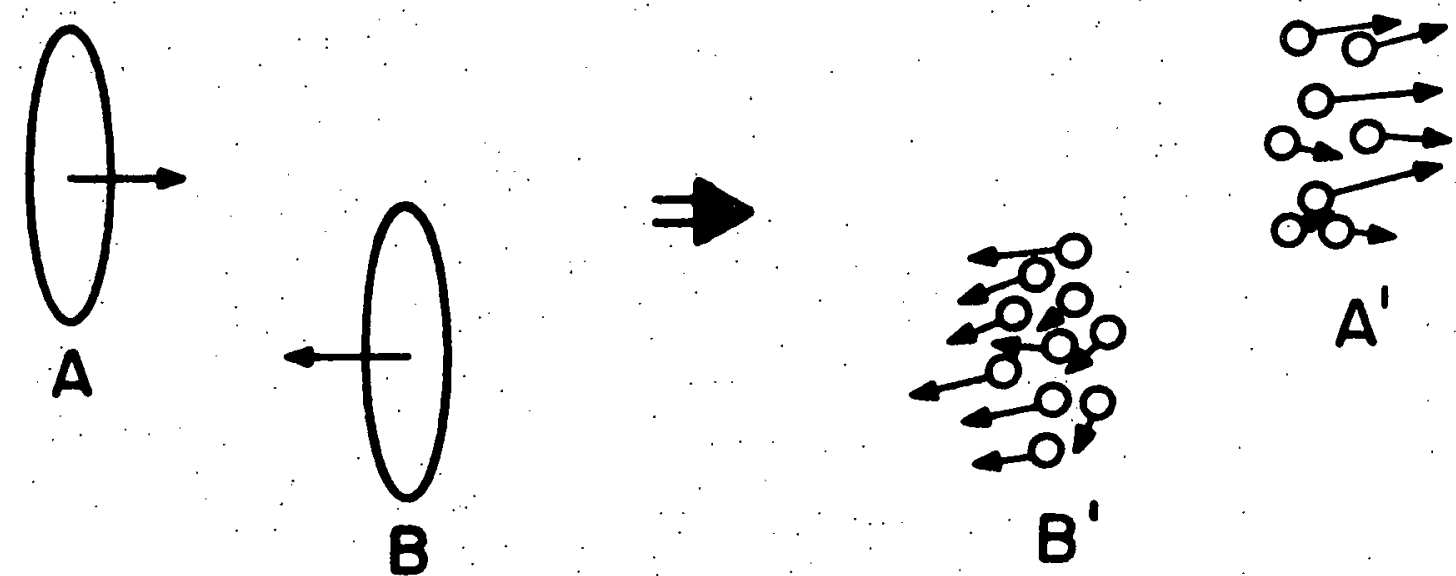

$A^{\prime}$

B
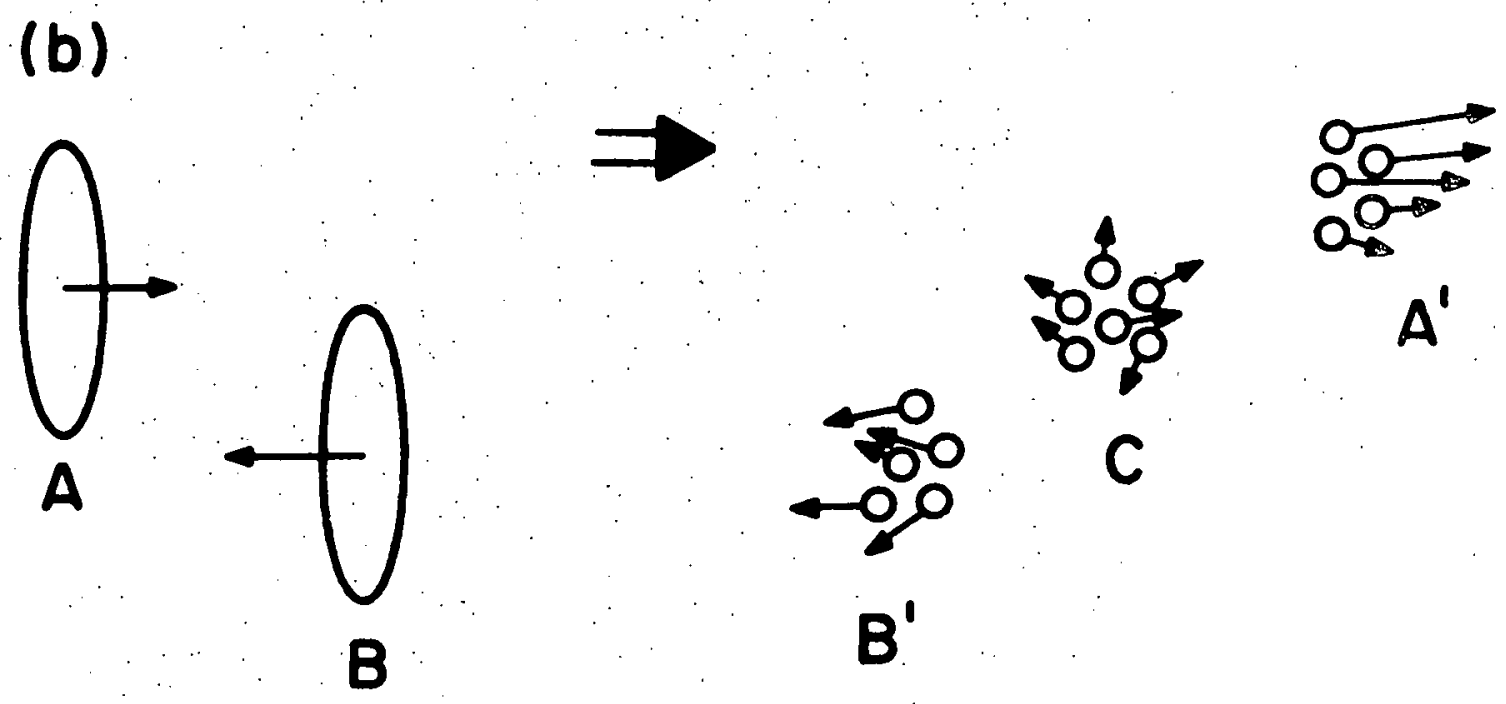

$A^{\prime}$

B'

Fig. 7 


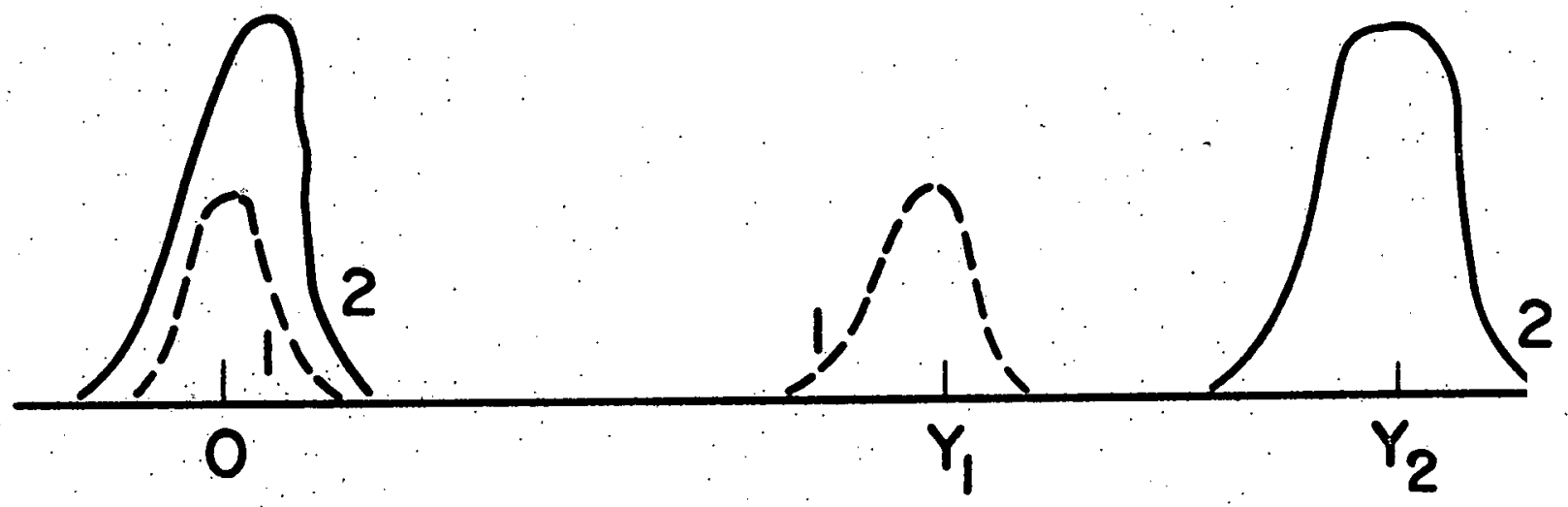

Fig. $8 a$

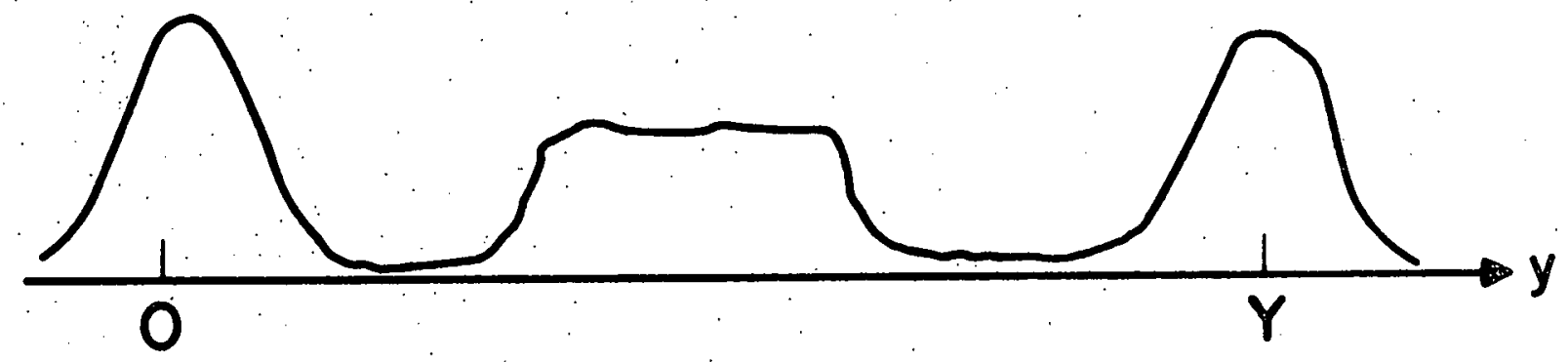

Fig. $8 b$

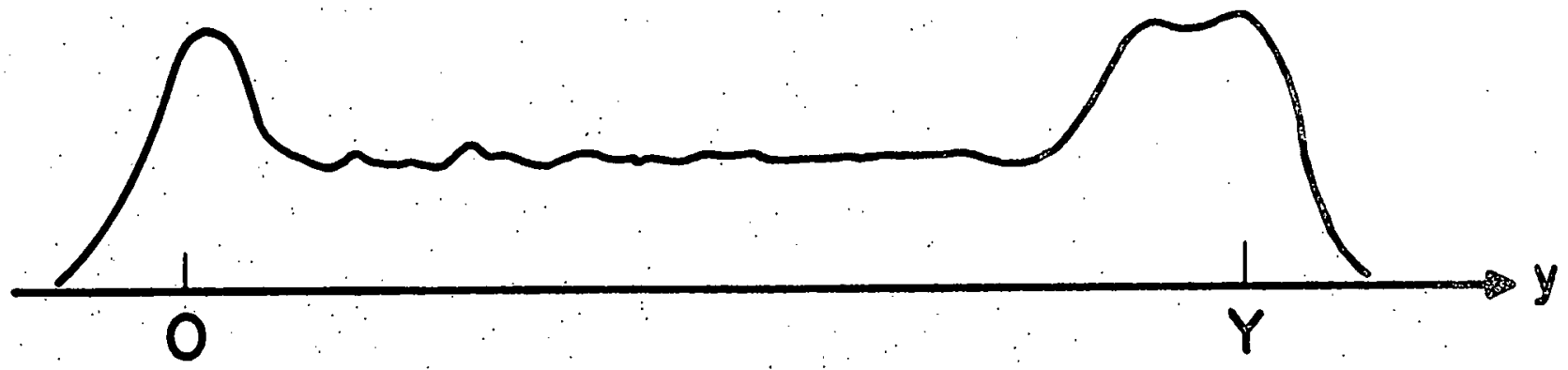

Fig. $8 \mathrm{C}$ 

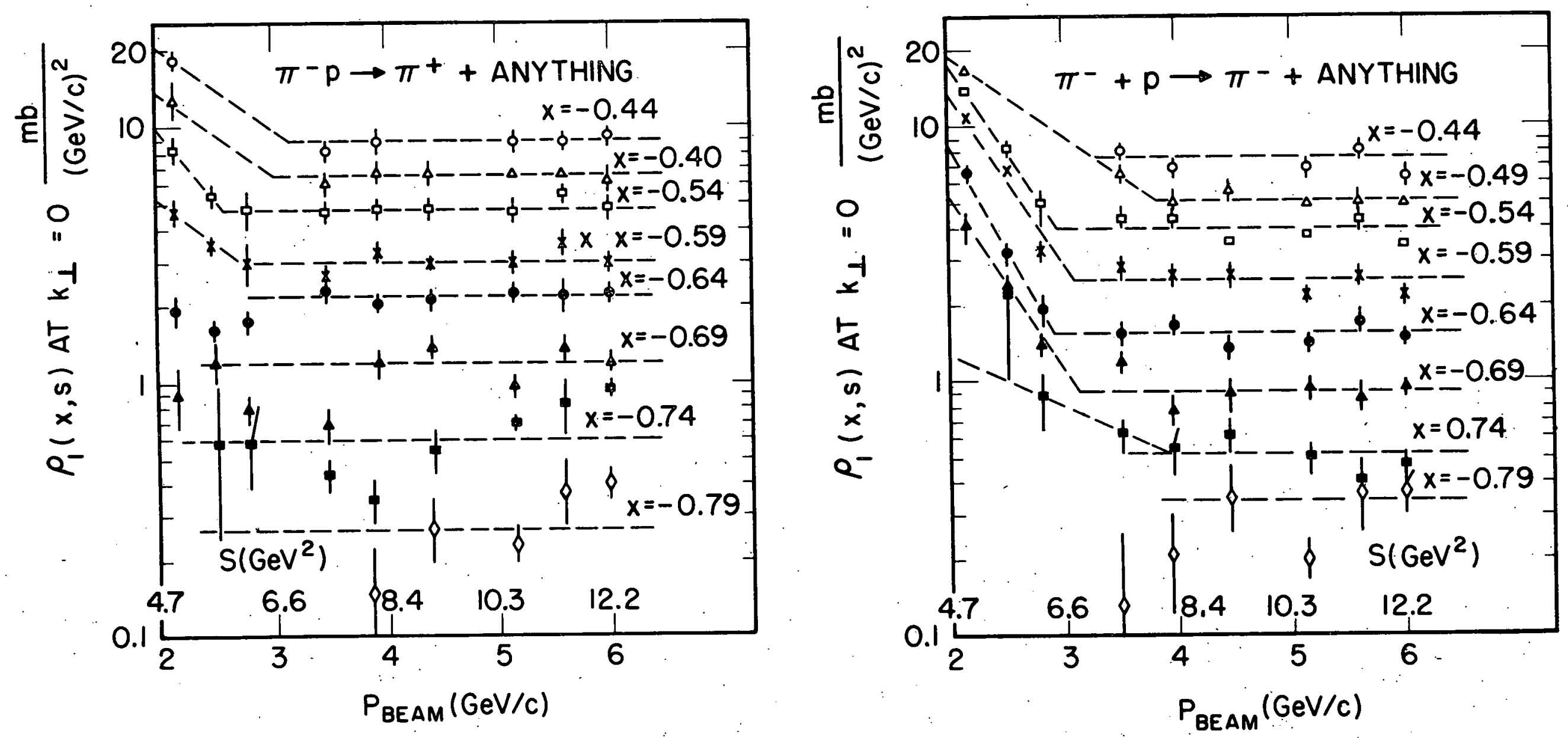

Fig. 9 


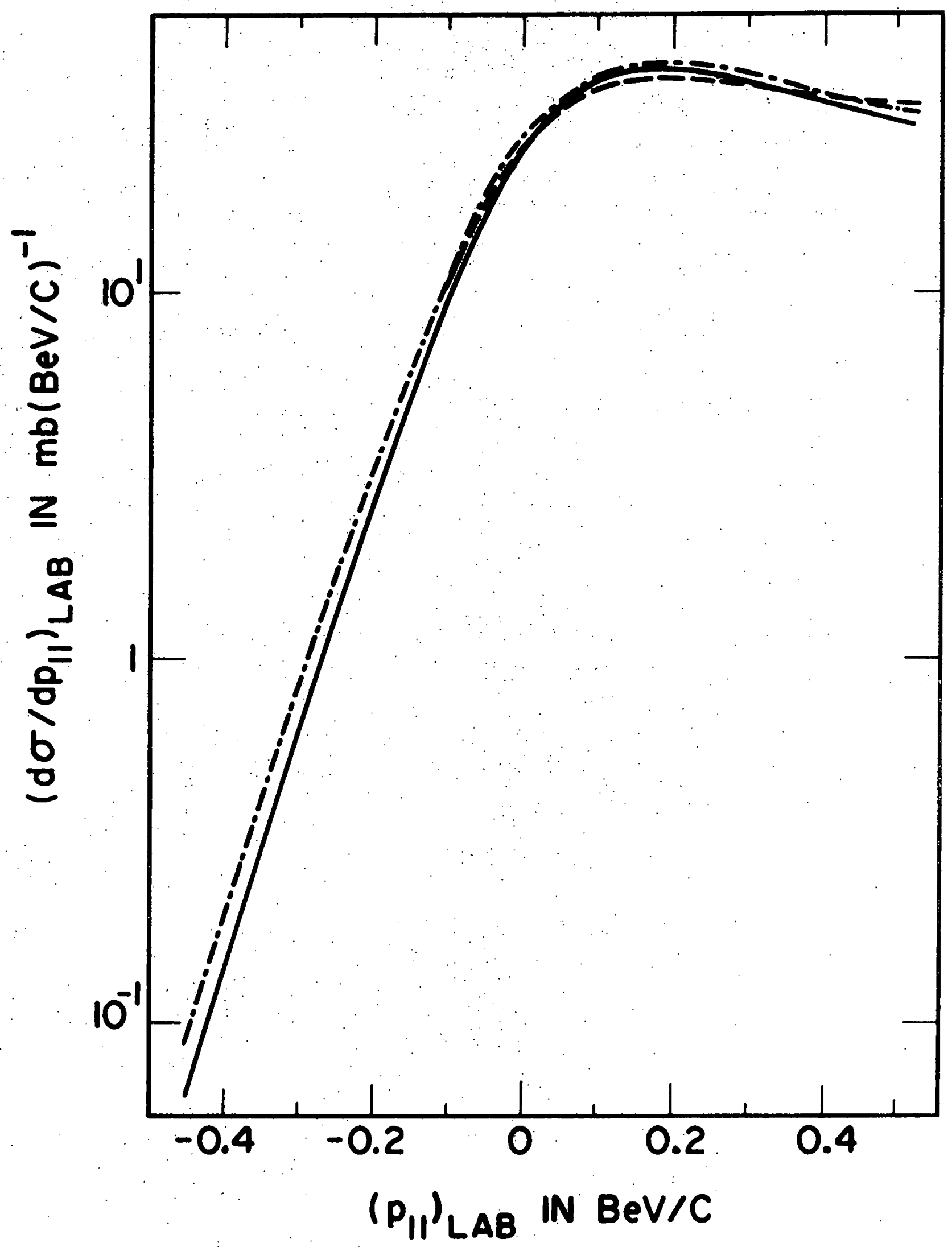

Fig. 10 


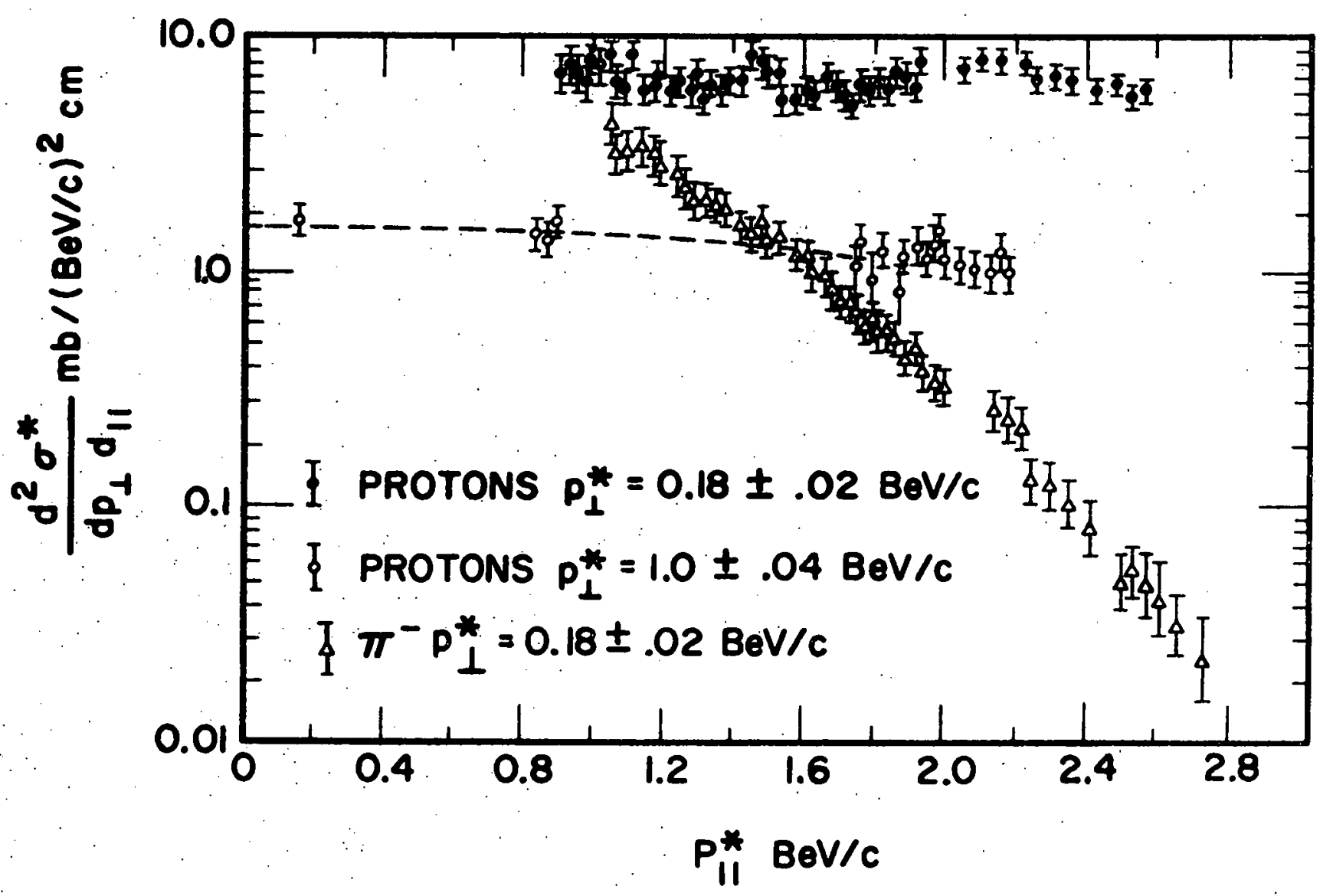

Fig. II 


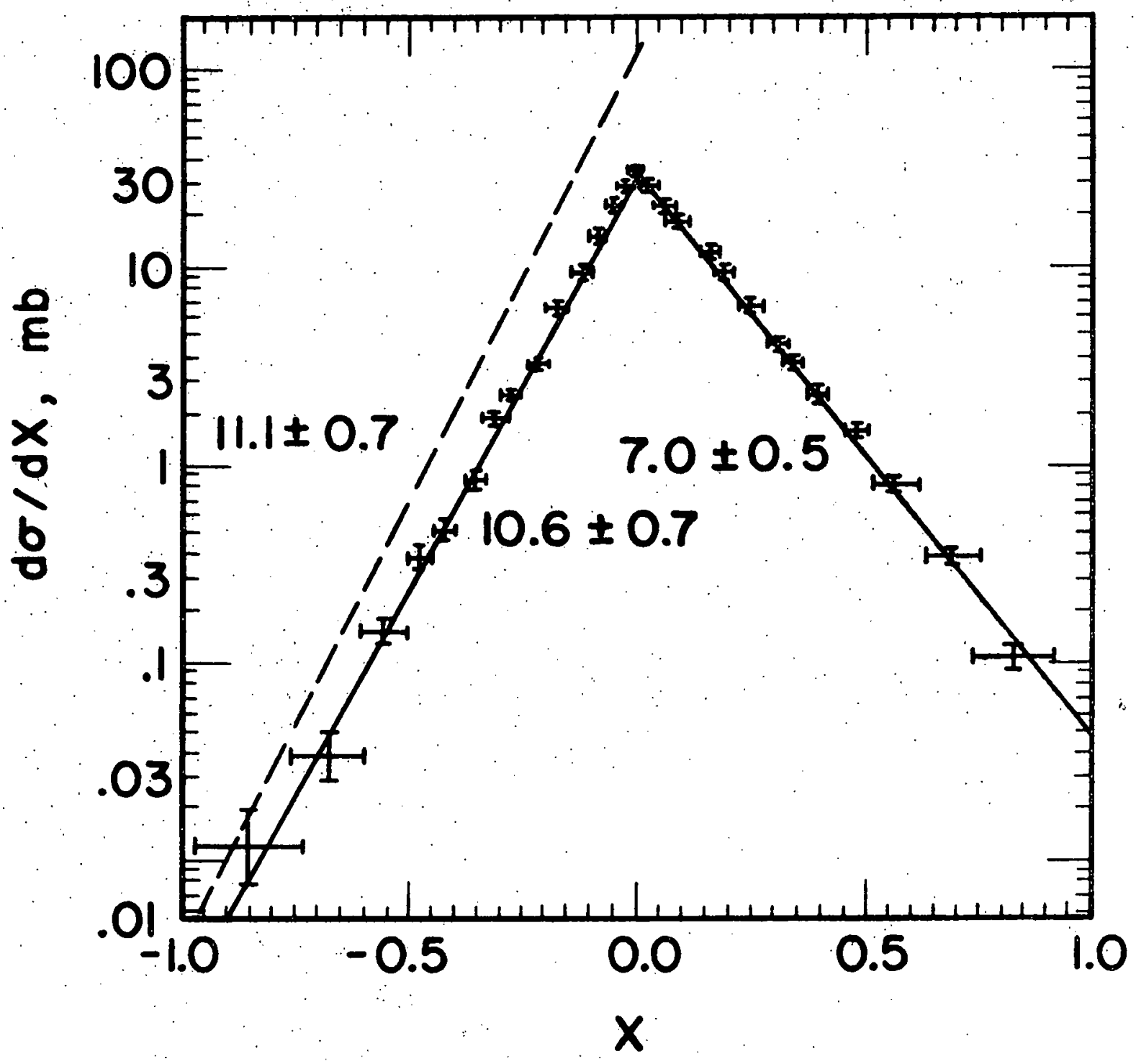

Fig. 12 

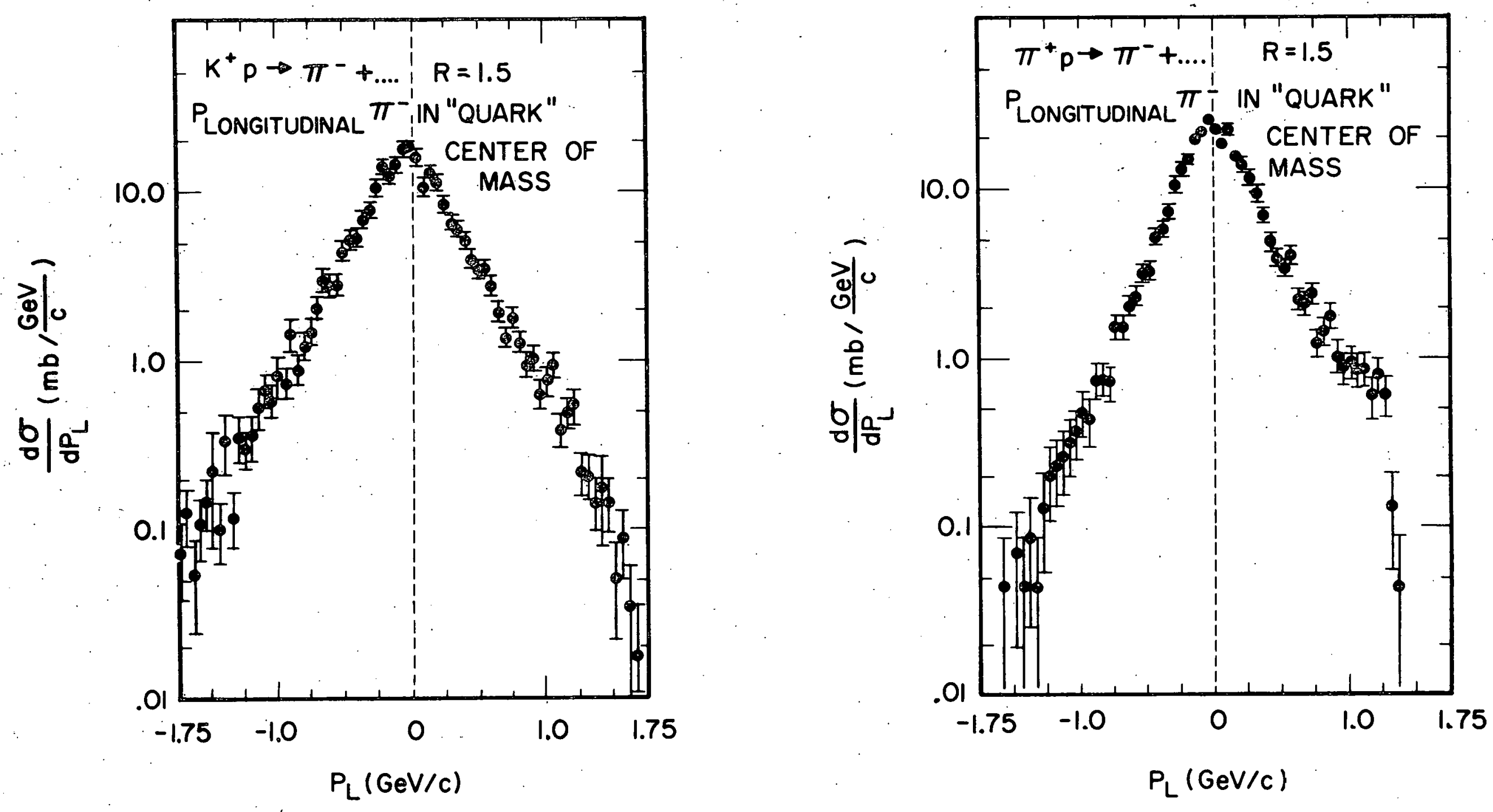

Fig. 13 


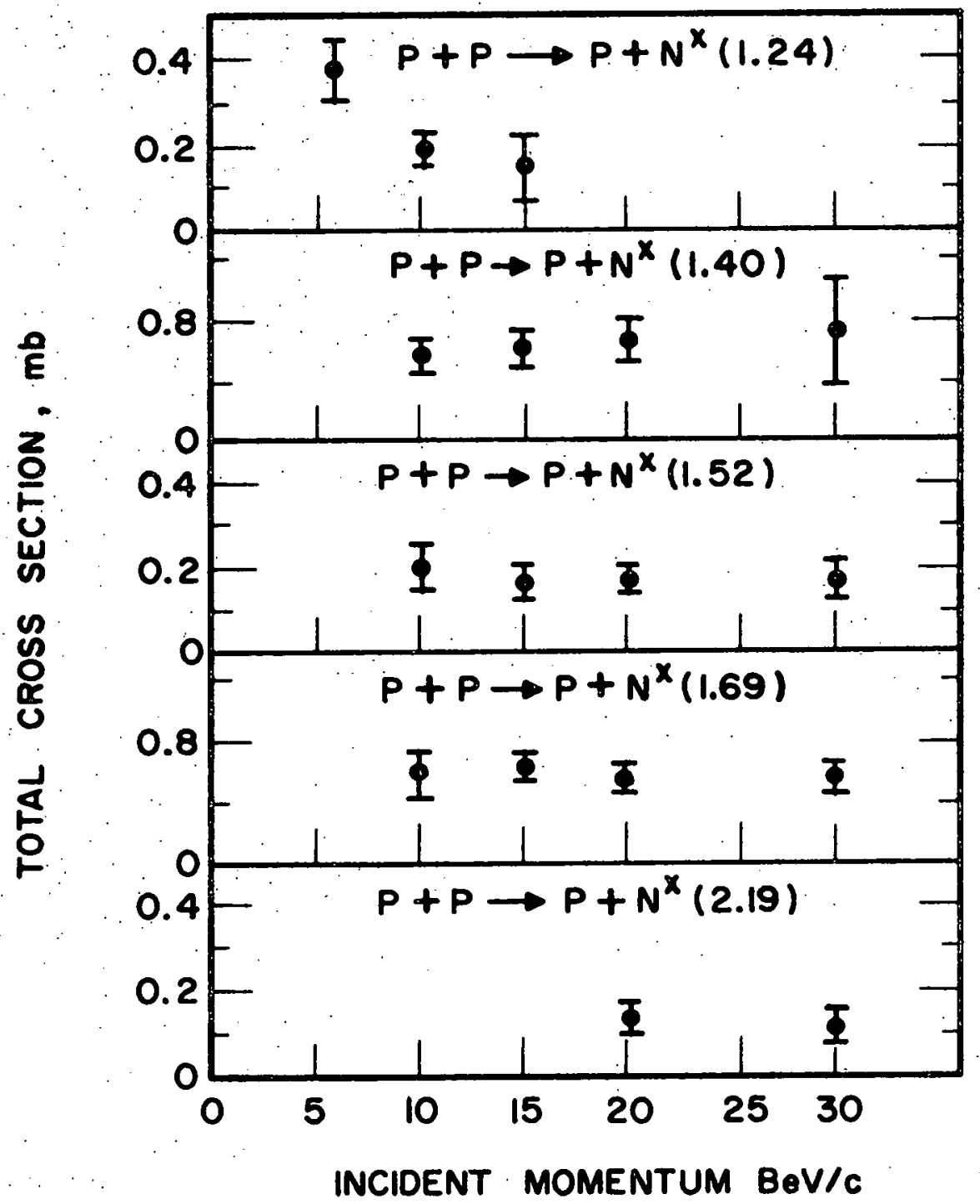

Fig. 14 


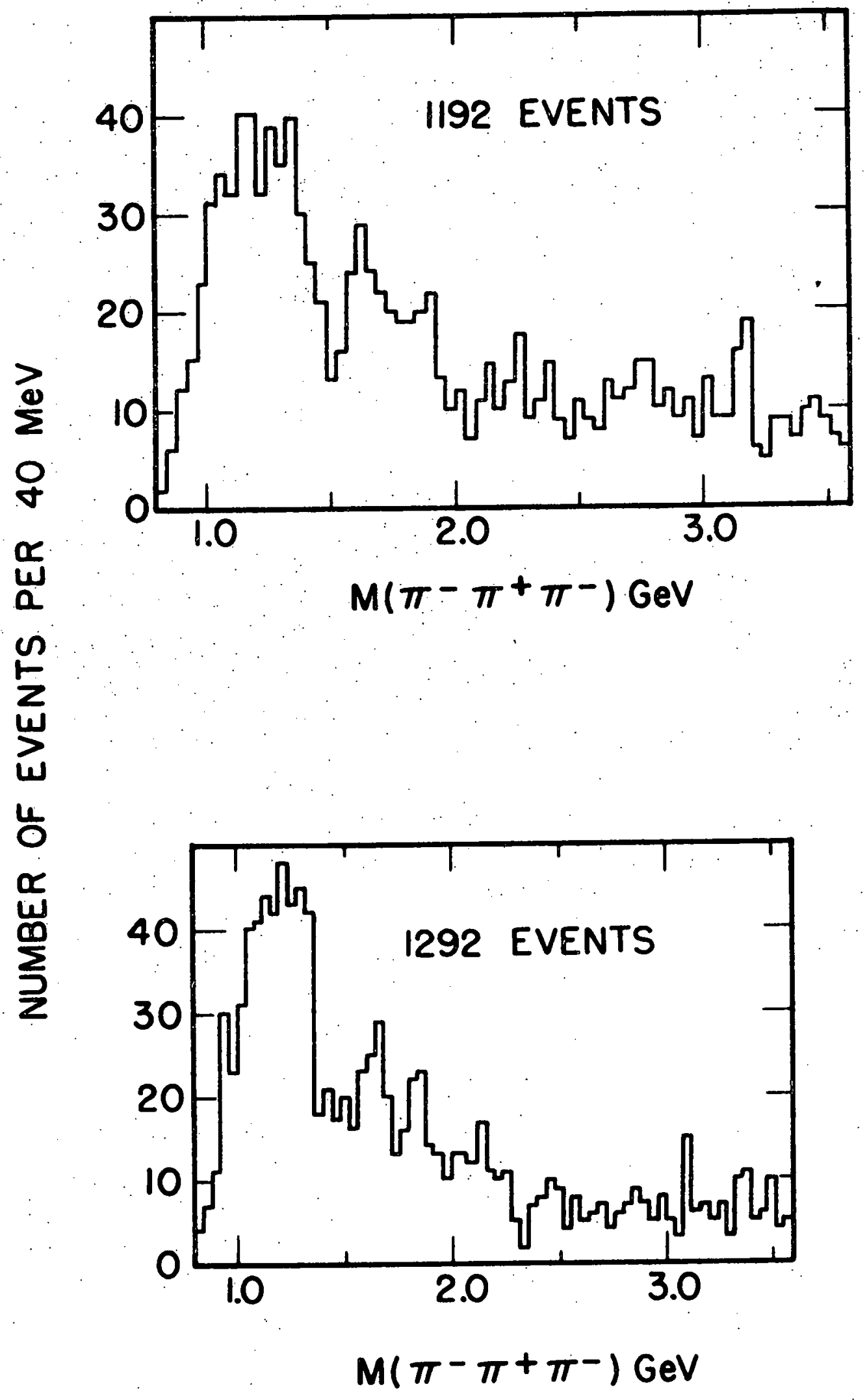

Fig. 15 


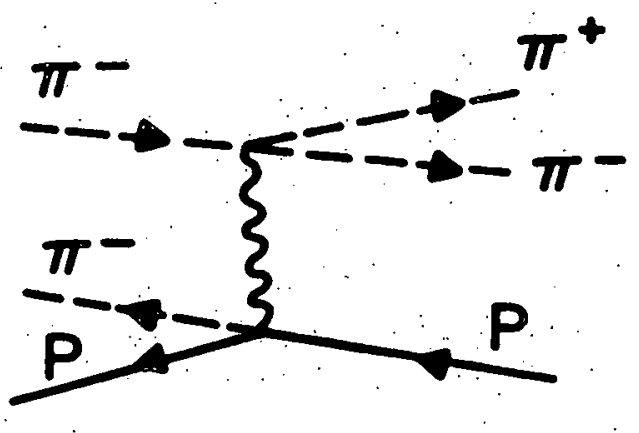

(a)

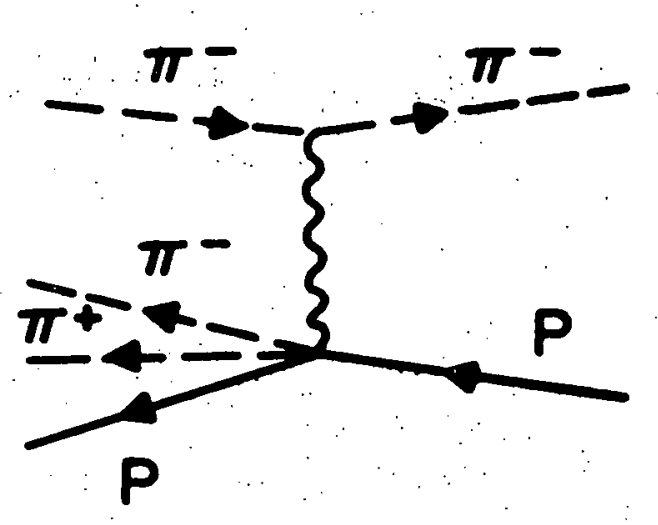

(c)

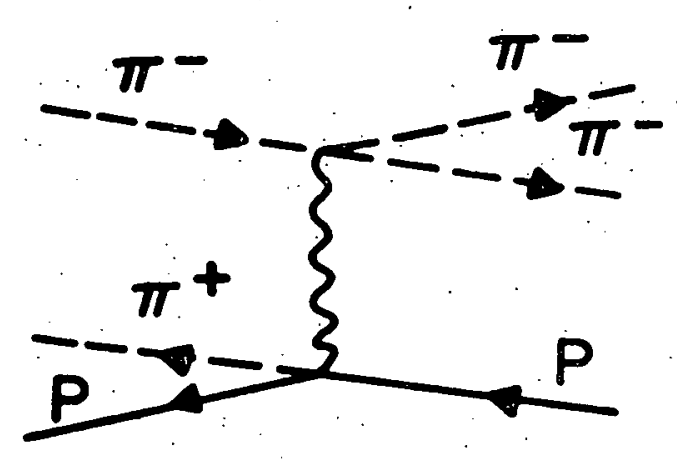

(b)

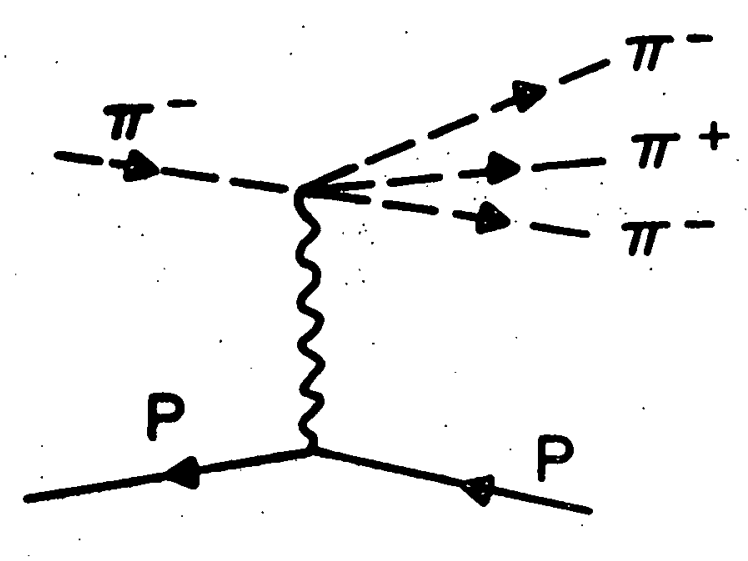

(d)

Fig. 16 


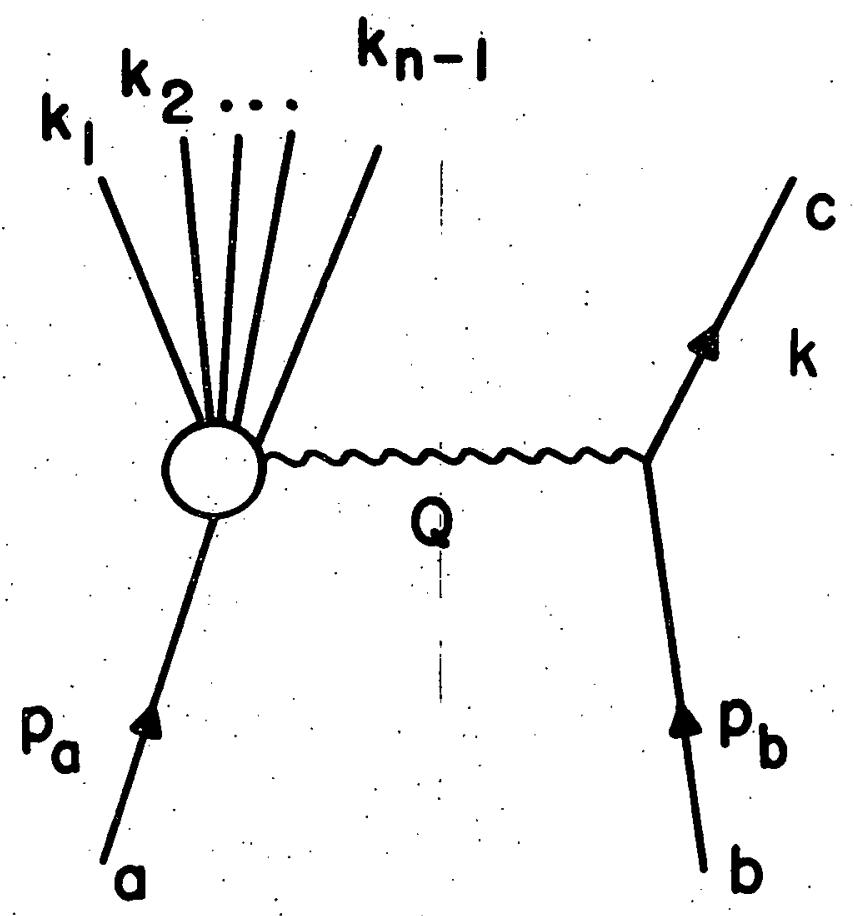

Fig. 17 

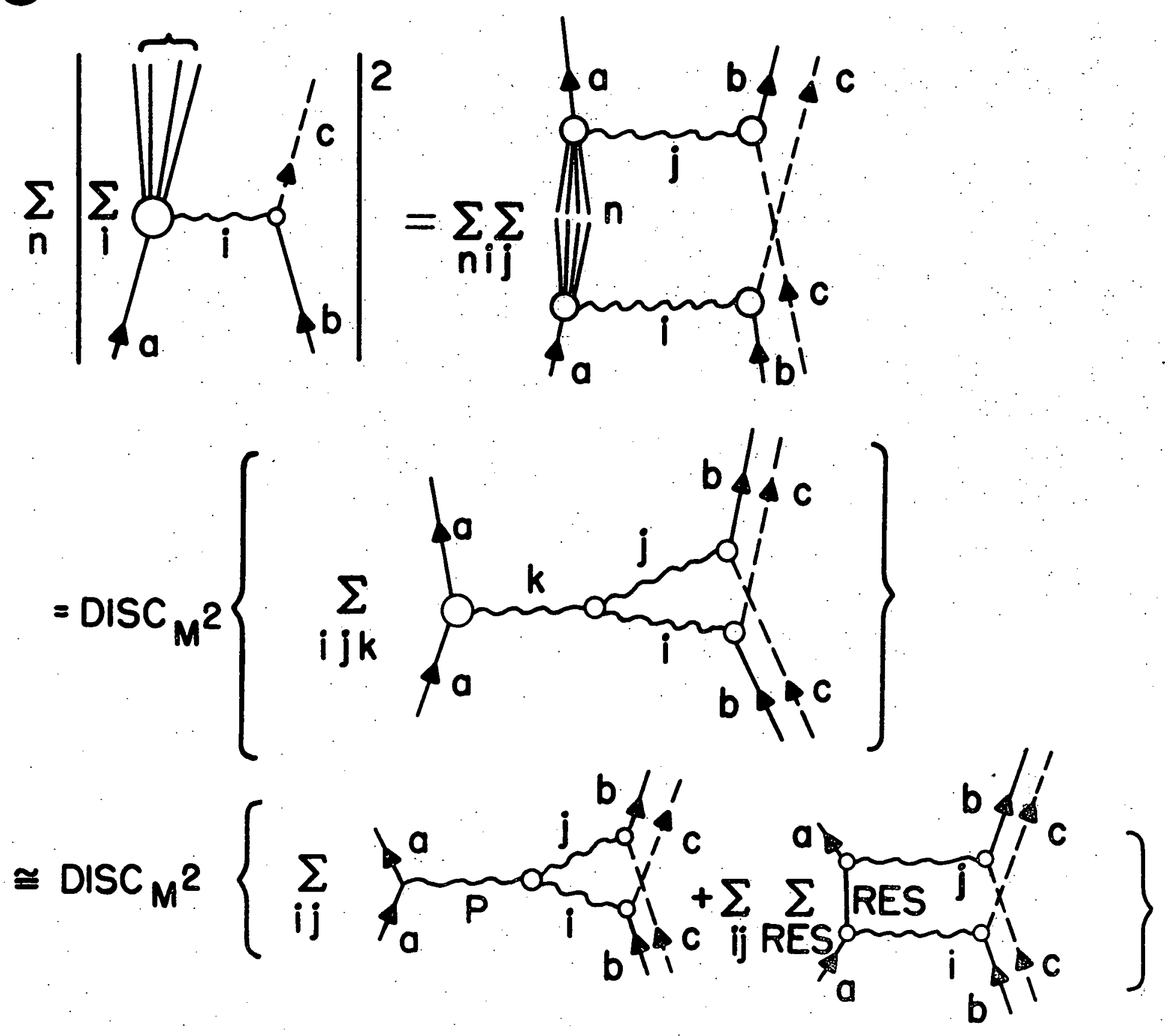

Fig. 18 


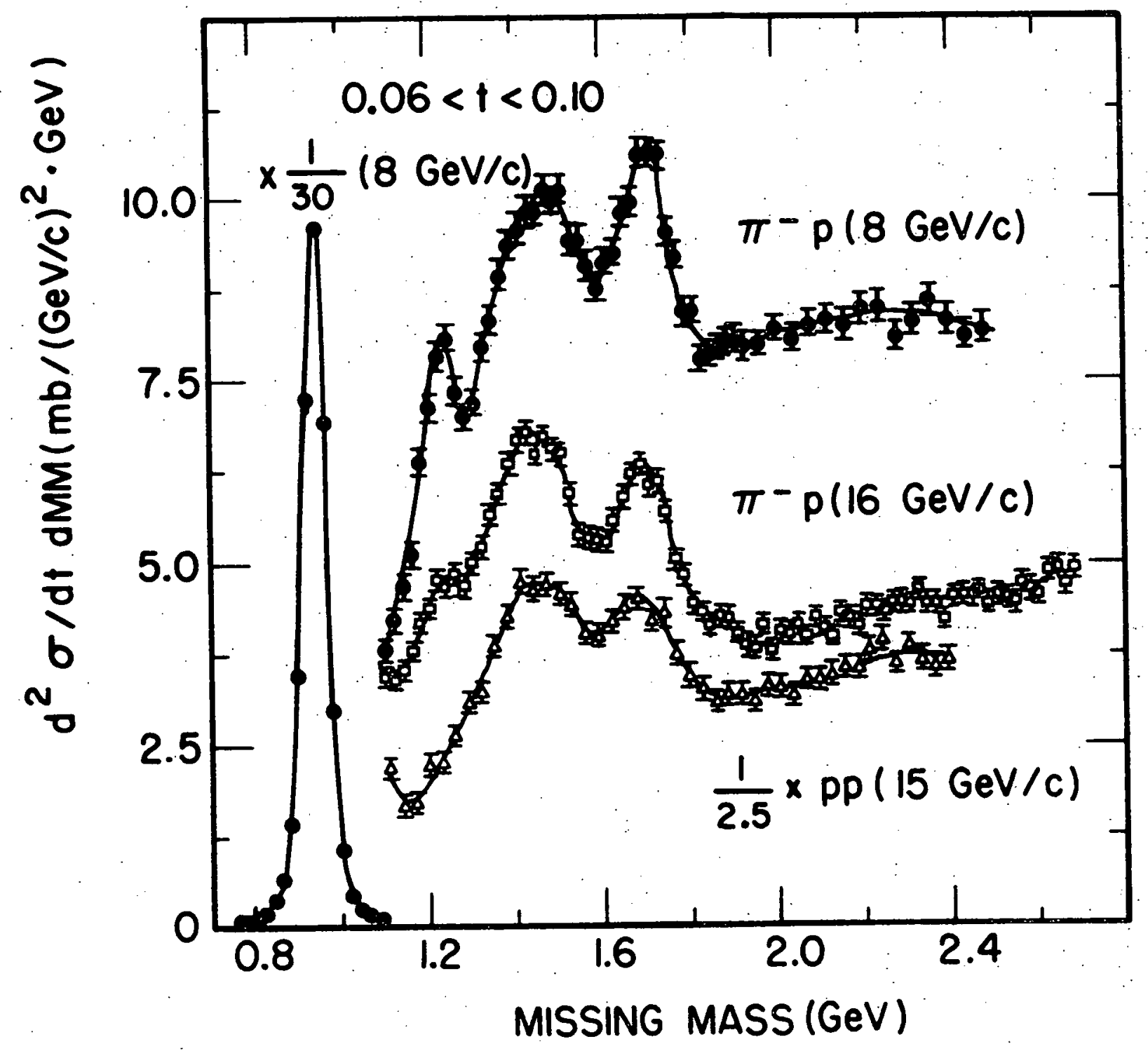

Fig. 19 


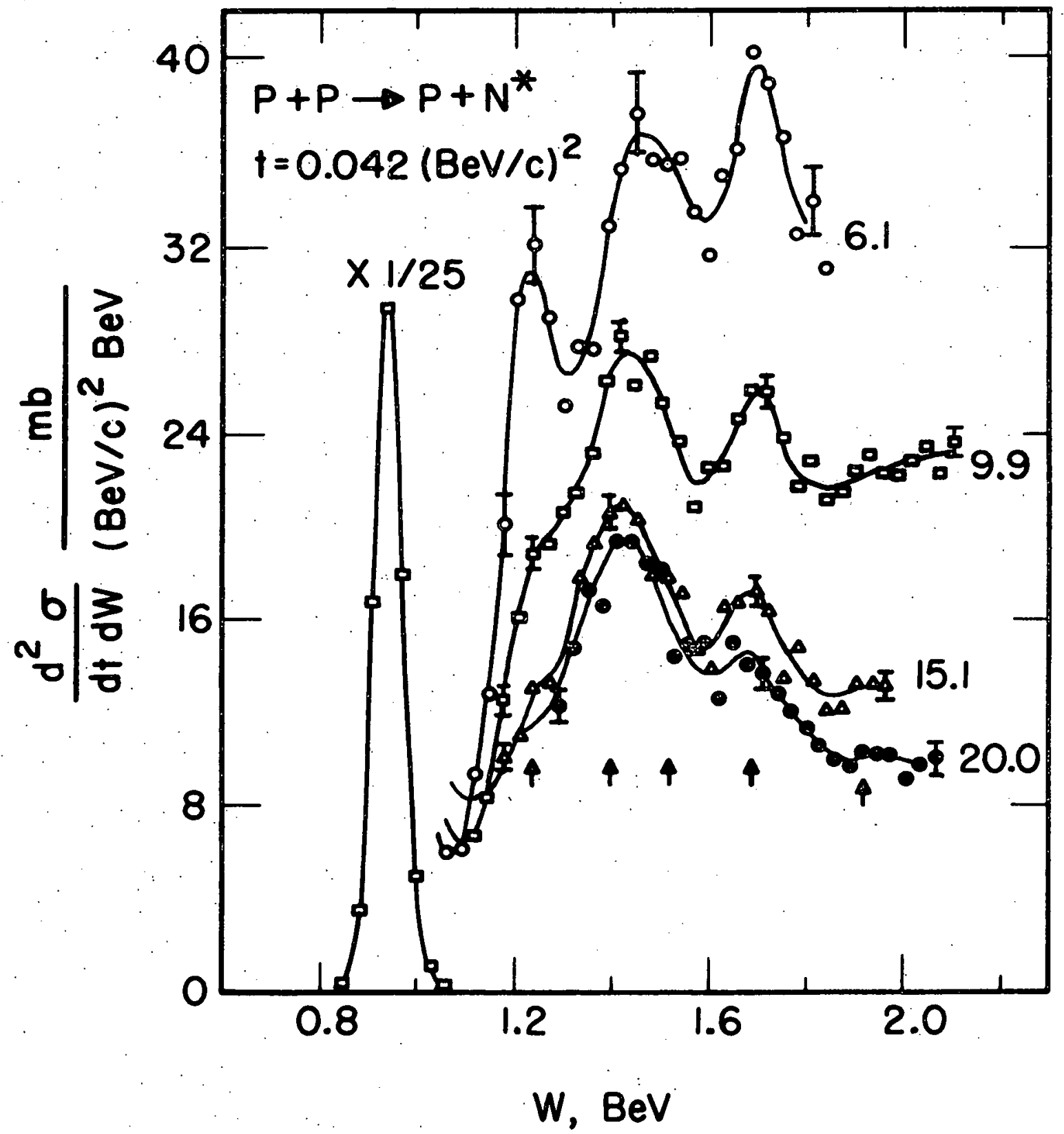

Fig. 20 


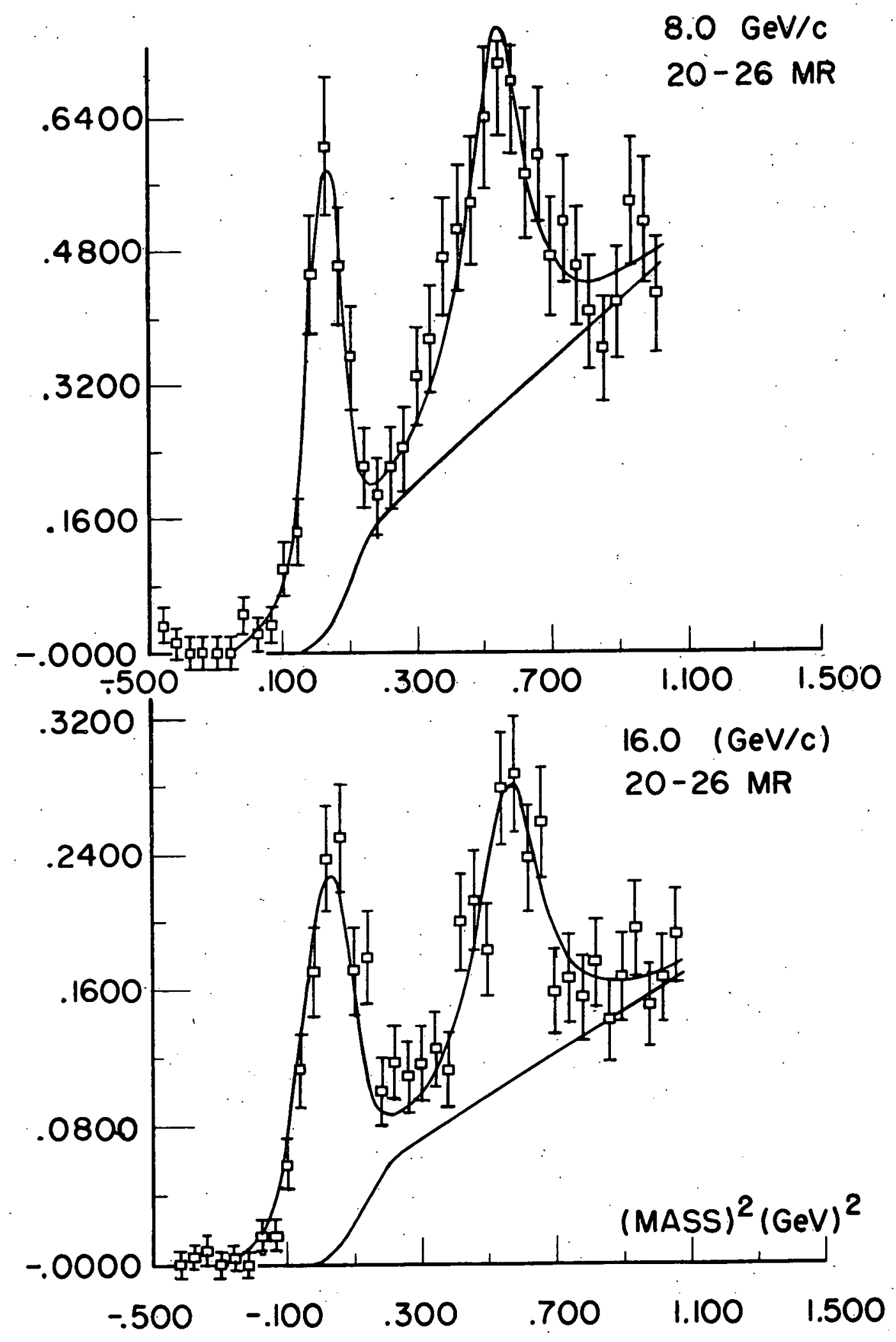

Fig. 21 


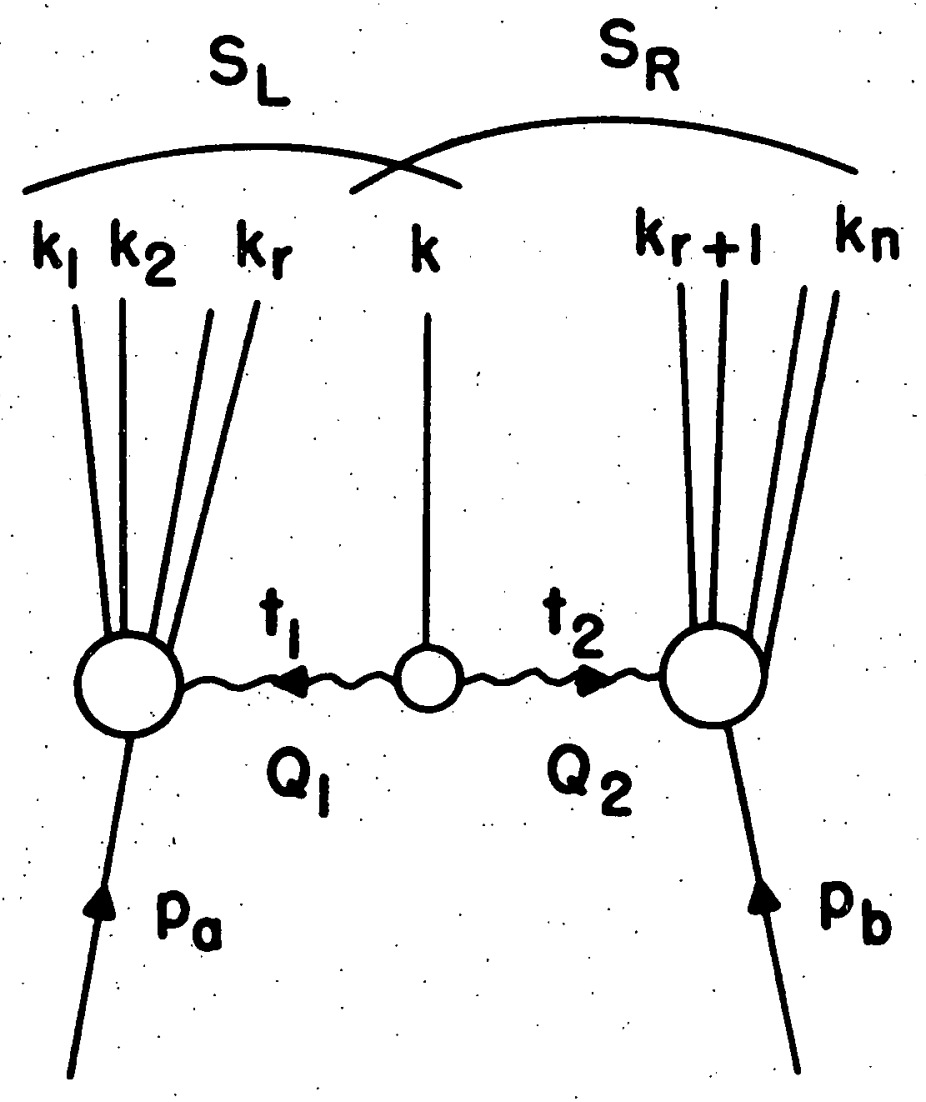

Fig. 22 


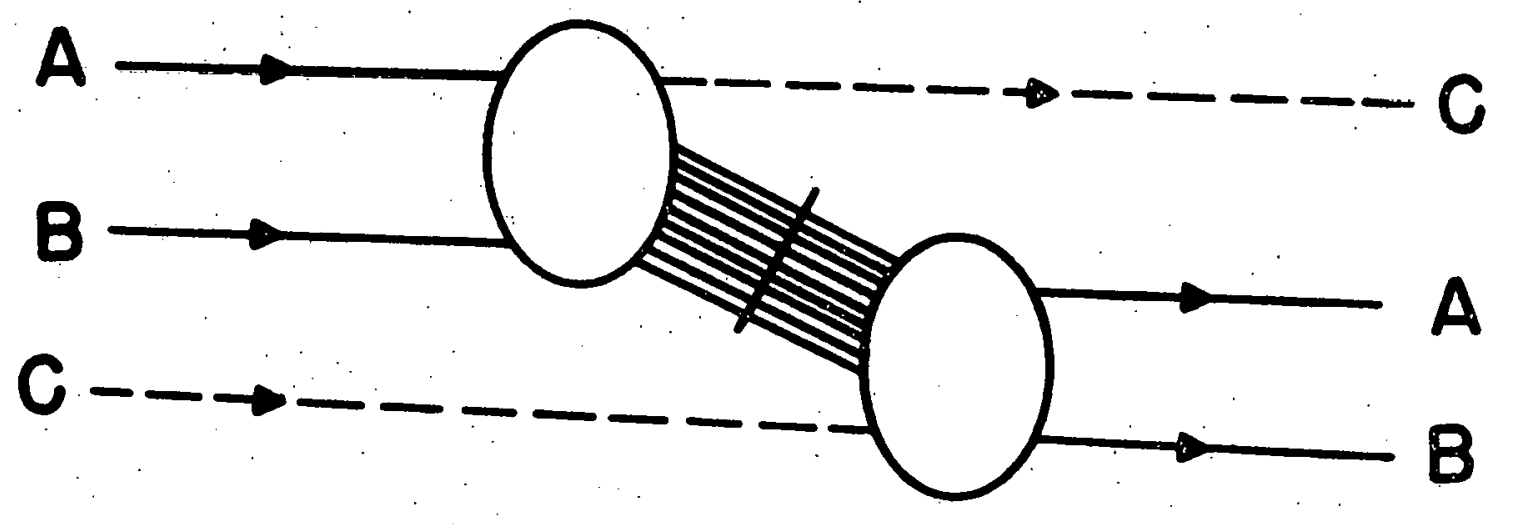

Fig. 23 